\title{
The NIST DMIS Interpreter
}

\author{
Thomas R. Kramer \\ Frederick M. Proctor \\ William G. Rippey \\ Harry Scott
}

Intelligent Systems Division

National Institute of Standards and Technology

Technology Administration

U.S. Department of Commerce

Gaithersburg, Maryland 20899

NISTIR 6012

April 24, 1997 


\section{Disclaimer}

No approval or endorsement of any commercial product by the National Institute of Standards and Technology is intended or implied.

\section{Acknowledgements}

Partial funding for the work described in this paper was provided to Catholic University by the National Institute of Standards and Technology under cooperative agreement Number 70NANB2H1213. 


\section{CONTENTS}

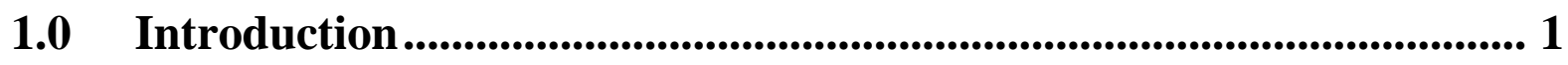

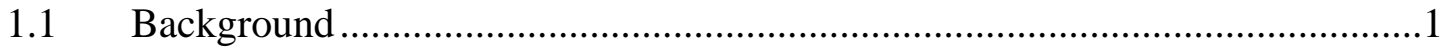

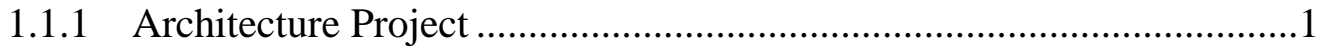

1.1.2 Enhanced Machine Controller Project ..................................................

1.1.3 Next Generation Inspection System Project ...................................

1.2 Overview of the DMIS Language .................................................................

1.2.1 Introduction...............................................................................

1.2.2 Statements, Lines, Major Words, Minor Words ................................2

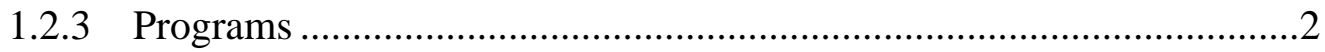

1.2.4 Program Subunits ....................................................................

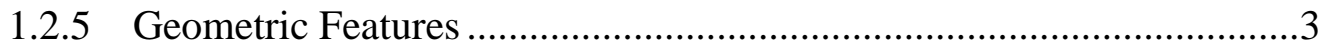

1.2.6 Tolerances .............................................................................. 3

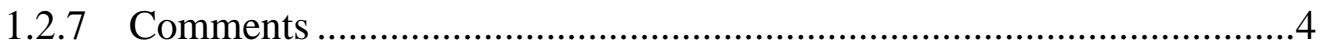

\subsection{Overview of the Interpreter ........................................................................... 4}

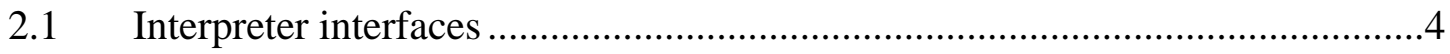

2.1.1 Telling the Interpreter What to Do................................................5

2.1.2 Getting Data from the Interpreter .................................................

2.1.3 Telling the CMM What to Do.............................................................5

2.1.4 Getting Data from the External World..............................................6

2.1.5 Extracting Feature Parameters from Arrays of Points ........................ 7

2.2 Integrated or Stand-Alone Operation ......................................................

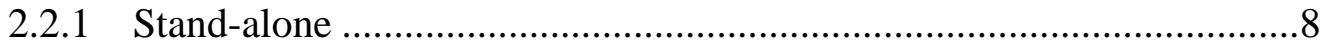

2.2.2 Integrated with EMC Control System..........................................

2.3 Major DMIS Interpreter Design Decisions ............................................. 10

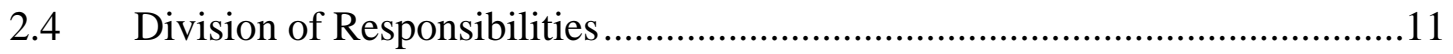

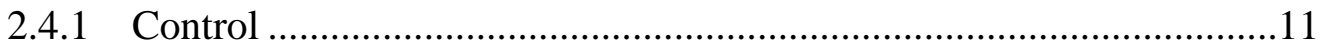

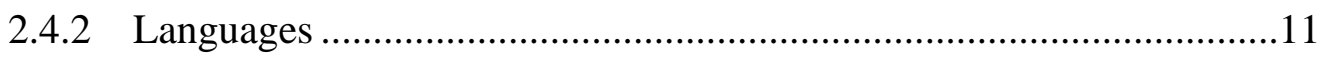

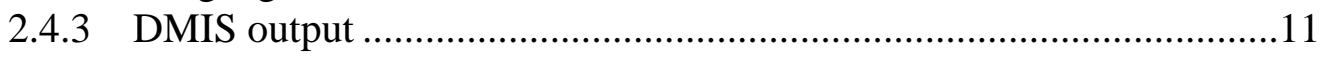

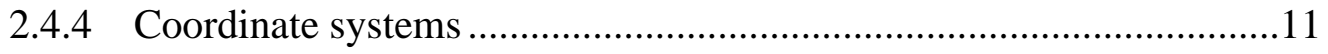

2.4.5 Features and Tolerances.............................................................11

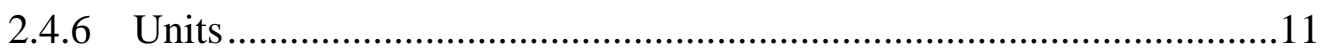

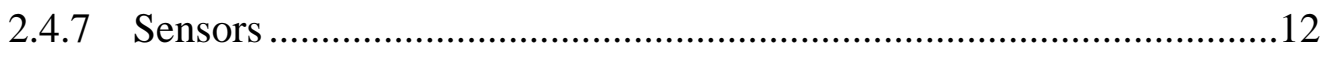

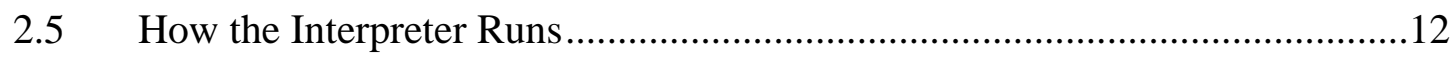

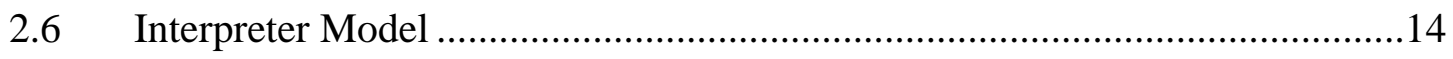

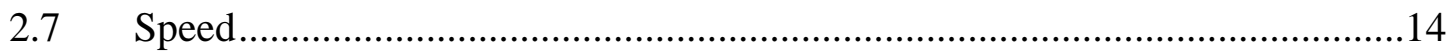


2.8 Limitations of the Interpreter

3.0 Input

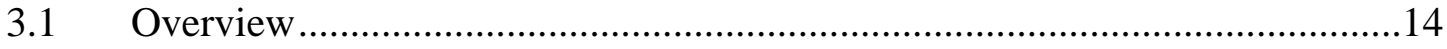

3.1.1 Case, White Space, Line Continuations, Comments.........................14

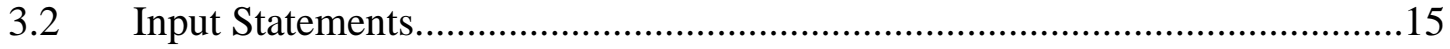

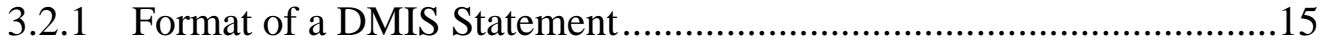

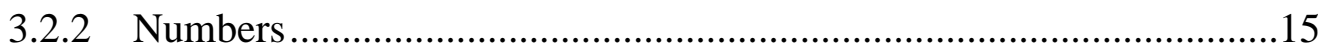

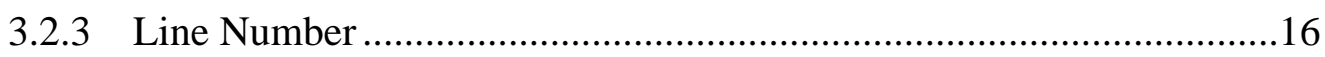

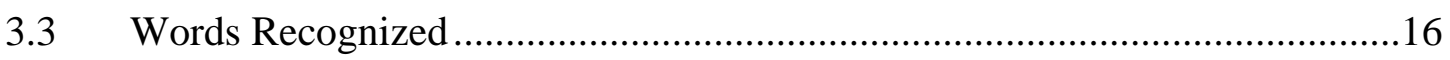

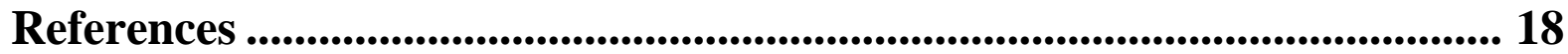

Appendix A Software Details .................................................................................... 19

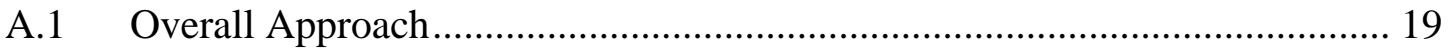

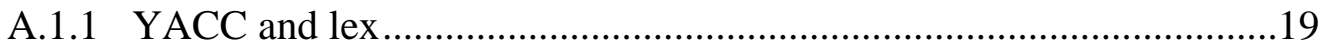

A.1.2 Read All First ............................................................................19

A.1.3 EXPRESS ..........................................................................19

A.1.4 YACC and EXPRESS .........................................................20

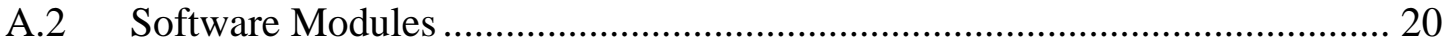

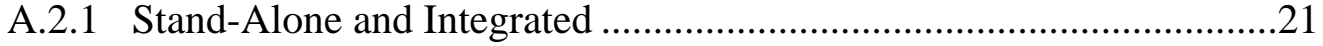

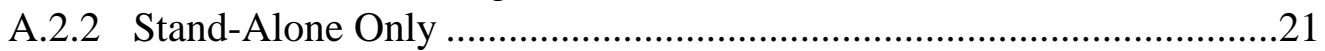

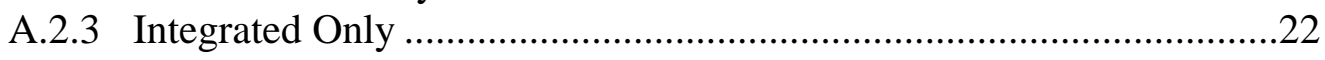

A.3 Source Code Documentation .............................................................. 22

Appendix B Interpreter Interface Functions ............................................... 23

B.1 Functions Which Extract Data From the Interpreter.................................. 23

B.2 Functions for the Interpreter to Call to Get World Model Data .................... 23

B.3 Functions to Tell the Interpreter What to Do............................................. 24

B.4 Functions to Tell the Rest of the System What to Do.................................. 26

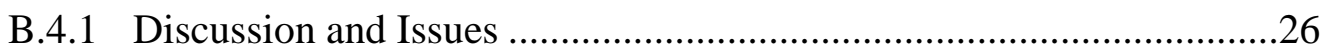

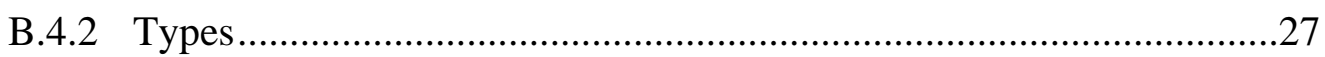

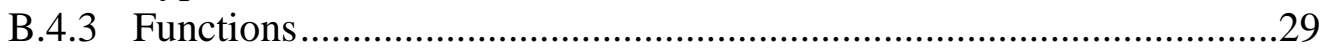

B.5 Functions to Get Feature Parameters from Arrays of Points........................ 39

Appendix C Building a Stand-Alone Executable ........................................... 42 
Appendix D Transcript of a Session ...................................................................... 44

Appendix E Error Handling and Error Messages.................................... 45

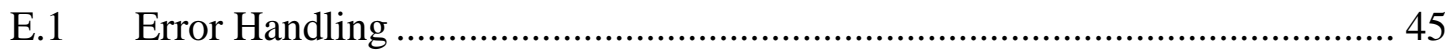

E.2 Types of Error Messages ................................................................... 45

E.2.1 Parser Error Messages...........................................................45

E.2.2 Kernel Error Messages.................................................................46

E.2.3 STEPTools Error Messages ..............................................................46

E.2.4 Driver Error Messages ...........................................................46

E.2.5 Feature Extraction Error Messages ............................................46

E.2.6 Interp_do_something Error Messages ..........................................46

E.3 Interpreter Kernel Error Messages....................................................... 46

Appendix F EXPRESS Schema for DMIS ..................................................... 50

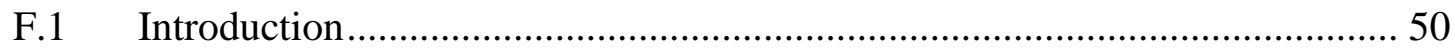

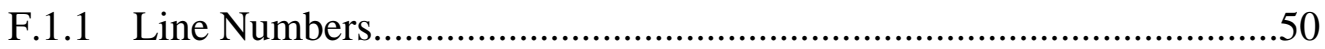

F.1.2 Optional Reals..........................................................................50

F.1.3 Actual Features .........................................................................50

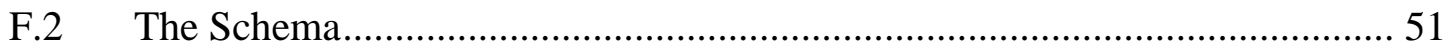

Appendix G YACC Tokens and Grammar Rules....................................... 70 
NIST DMIS Interpreter 


\section{FIGURES}

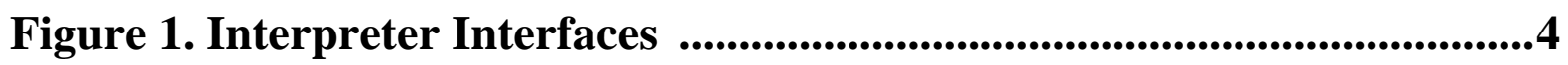

Figure 2. Stand-Alone Interpreter ............................................................................9

Figure 3. Interpreter Integrated in Controller ..............................................10 


\section{TABLES}

Table 1. CMM Canonical Commands.......................................................................... 6

Table 2. Interpreter Internal Model .......................................................... 13

Table 3. DMIS Words Implemented in the Interpreter ................................ 17

Table 4. Interpreter State Transitions.............................................................. 25

Table 5. Makefile for Interpreter........................................................................... 43 


\section{Introduction}

The NIST DMIS interpreter is a software system which reads control code programs in the DMIS language (described below in Section 1.2), produces calls to a set of canonical commands for coordinate measuring machines, digests the results of taking measurements, and produces a file describing measured features and tolerances. The canonical command calls made by the interpreter can be used to drive a coordinate measuring machine. This report describes the DMIS interpreter (in this report "the interpreter").

\subsection{Background}

\subsubsection{Architecture Project}

The NIST Manufacturing Engineering Laboratory (MEL) has conducted an architecture project for several years. Three MEL divisions have participated in the project. The primary objective of the project is to develop a reference model control architecture to support intelligent control systems for manufacturing. The architecture being developed is called the Intelligent Systems Architecture for Manufacturing (ISAM) [Albus].

\subsubsection{Enhanced Machine Controller Project}

The MEL Intelligent Systems Division (ISD) is carrying out an Enhanced Machine Controller (EMC) project. The primary objective of the EMC project is to build a testbed for evaluating application programming interface standards for open-architecture machine controllers. The EMC project has built several controllers. These are most often run in a research environment at NIST, but commercial installations of EMC controllers have also been done [Proctor].

\subsubsection{Next Generation Inspection System Project}

To advance the state of the art in inspection, ISD established the Next Generation Inspection System (NGIS) project. NGIS goals are to maintain a next generation inspection testbed for experimenting with open architecture controllers, interface standards, and multiple advanced sensors, and to achieve fast, accurate, and flexible coordinate measurement of complex parts. A testbed has been assembled that consists of a coordinate measuring machine and advanced sensors, with a NIST Real Time Control System (RCS) architecture controller.

As part of the architecture project, it was decided to put two levels of EMC controllers above the NGIS controller. The capability to interpret control programs was put in the control level immediately above the NGIS controller. DMIS was selected as the language for control programs.

\subsection{Overview of the DMIS Language}

This section gives an overview of the DMIS language. Further details of the meaning of DMIS code are given in Section 3 of this report.

\subsubsection{Introduction}

DMIS (pronounced DEE-miss and standing for Dimensional Measuring Interface Standard) is a standard programming language for numerically controlled dimensional measuring equipment, primarily coordinate measuring machines (CMMs). Coordinate measuring machines from many manufacturers can be operated using programs written in DMIS.

DMIS was developed by the Consortium for Advanced Manufacturing - International. The most 
recent version of DMIS is Revision 3.0, which was completed in 1995 [CAM-I] and is ANSI American National Standard "ANSI/CAM-I 101-1995." The interpreter conforms to Revision 3.0.

The DMIS specification [CAM-I] is large - 389 pages. It describes both an input language and an output language. The DMIS input language supports the following functions:

defining and measuring features (planes, circles, cylinders, lines, etc.)

defining and measuring tolerances

defining coordinate systems (and activating and deactivating them)

defining sensor characteristics and changing sensors

setting machine parameters (feed rates, probe tip radius, etc.)

machine motion - probing and free-space motion

The output language supports reporting the results of measuring features and tolerances and also serves as a log of input statements.

The general outline of a typical DMIS program is to define and measure some features on a part which serve to establish the coordinate system in which further measurements will be taken. Then, more features and tolerances on and among features are defined and measured in the newly established coordinate system. The measurements are analyzed, actual tolerances are calculated, and the results are saved in a file.

\subsubsection{Statements, Lines, Major Words, Minor Words}

DMIS is based on statements. A statement normally fits on a single line (a series of ASCII characters terminated by a carriage return and line feed). However, lines may be continued by putting the line continuation symbol (the $\$$ character) as the last printable character on a line, so that a single statement may span several lines.

A typical statement consists of a major word, followed by a slash, followed by a mixture of minor words, labels, and numbers, for example MEAS/PLANE, F(POCKET_BTM), 3. Semantically, each statement represents a single command which is embodied in the major word. The minor words, the numbers, and the way in which the minor words and numbers are grouped specify parameters to the command and shades of meaning of the command.

The statement formats recognized by the interpreter are presented in a formal specification language in Appendix G. This includes about a quarter of the entire DMIS language, but it is the most heavily used quarter and covers perhaps 90 percent of what might be seen in typical inspection programs.

\subsubsection{Programs}

Statements may be collected in a file to make a program. A program consists of a DMISMN statement at the beginning ${ }^{1}$, an ENDFIL statement at the end, and any number of other types of statements in between. The specification is not clear whether statements are intended to be usable outside of a program (as manual data input, for example). The interpreter requires an entire program.

1. By "DMISMN statement" we mean a statement using the major word DMISMN. In general, "XYZ statement" means a statement whose major word is XYZ. 


\subsubsection{Program Subunits}

DMIS includes program subunits. A program subunit is a sequence of statements which forms a functional group. [CAM-I] defines ten types of program subunits. The interpreter implements only two of these types: measurement sequence and motion sequence. Each type of program subunit requires a particular type of first statement and a particular type of last statement.

A measurement sequence has a MEAS statement at the beginning and an ENDMES statement at the end. The function of a measurement sequence is to measure one feature. The significant statements inside a measurement sequence are PTMEAS statements, each of which is a command to measure a point.

A motion sequence has a GOTARG at the beginning and an ENDGO at the end. The function of a motion sequence is to move around in free space. Only GOTO statements may occur inside a motion sequence.

In the interpreter, we use the word "block" to mean either a statement or a program subunit.

\subsubsection{Geometric Features}

In DMIS, inspecting a part is done in terms of features and tolerances. Features in DMIS are mostly simple geometric elements. A complete list of DMIS feature types is: arc, circle, cone, cparln, cylinder, ellipse, gcurve, gsurf, line, object, parpln pattern, plane, point, rectangle, and sphere. The underlined five are implemented in the interpreter. DMIS features (such as the cylindrical side of a hole) may be visible on a part being inspected or they may be purely conceptual (such as the line which is the axis of a cylindrical hole).

A DMIS program usually does not try to provide a complete description of the part to be inspected. Only those features which are to be measured or used indirectly for definitions need to be defined. There is no requirement on how much of the geometry of a feature must be present. For example, a line joining the centers of two circles is common in a DMIS program, even though there is no trace of it on the actual part.

DMIS does not provide a general geometric modeling capability. DMIS provides no capability to describe topology and no capability to perform modeling operations such as boolean subtraction of a feature from a part.

Each feature is considered to have both a nominal description, which is the one used when the feature is first defined, and an actual description, which is derived later on the basis of one or more measurements. The DMIS specification does not state whether a single nominal feature may correspond to more than one actual feature, but seems to assume that the correspondence is oneto-one.

Each feature has a label which serves to identify it within a DMIS program. No other feature may share that label in the same program.

\subsubsection{Tolerances}

DMIS tolerances also have labels which are unique among tolerances within a program.

Tolerances in DMIS do not belong to individual features. Tolerances are defined without reference to specific features and may be applied repeatedly. For example, a diameter tolerance of 0.1 millimeter might be defined and labelled DTOL1. Then a dozen circles might be tested to see if 
they meet DTOL1.

DMIS supports tolerances according to the ASME Y14.5-1994 Standard for Dimensioning and Tolerancing. Twenty-two types of tolerance are included. The interpreter implements seven of these to one degree or another: coordinate position, cylindricity, diameter, flatness, parallelism, perpendicularity, and relative position.

\subsubsection{Comments}

A DMIS program may include comments. A comment is a line which has two dollar signs as the first two characters. Such lines are to be ignored by the system executing DMIS statements. Comments may contain information useful to humans writing or using the program.

\section{Overview of the Interpreter}

\subsection{Interpreter interfaces}

The interpreter has five interfaces, as shown in Figure 1. Each interface is a collection of function calls; there is an application programming interface (API) for each such collection. Arrows show the direction of function calls. Return values (shown in parentheses) move in the reverse direction. cmm_do_something calls do not return anything, so nothing goes in the reverse direction.

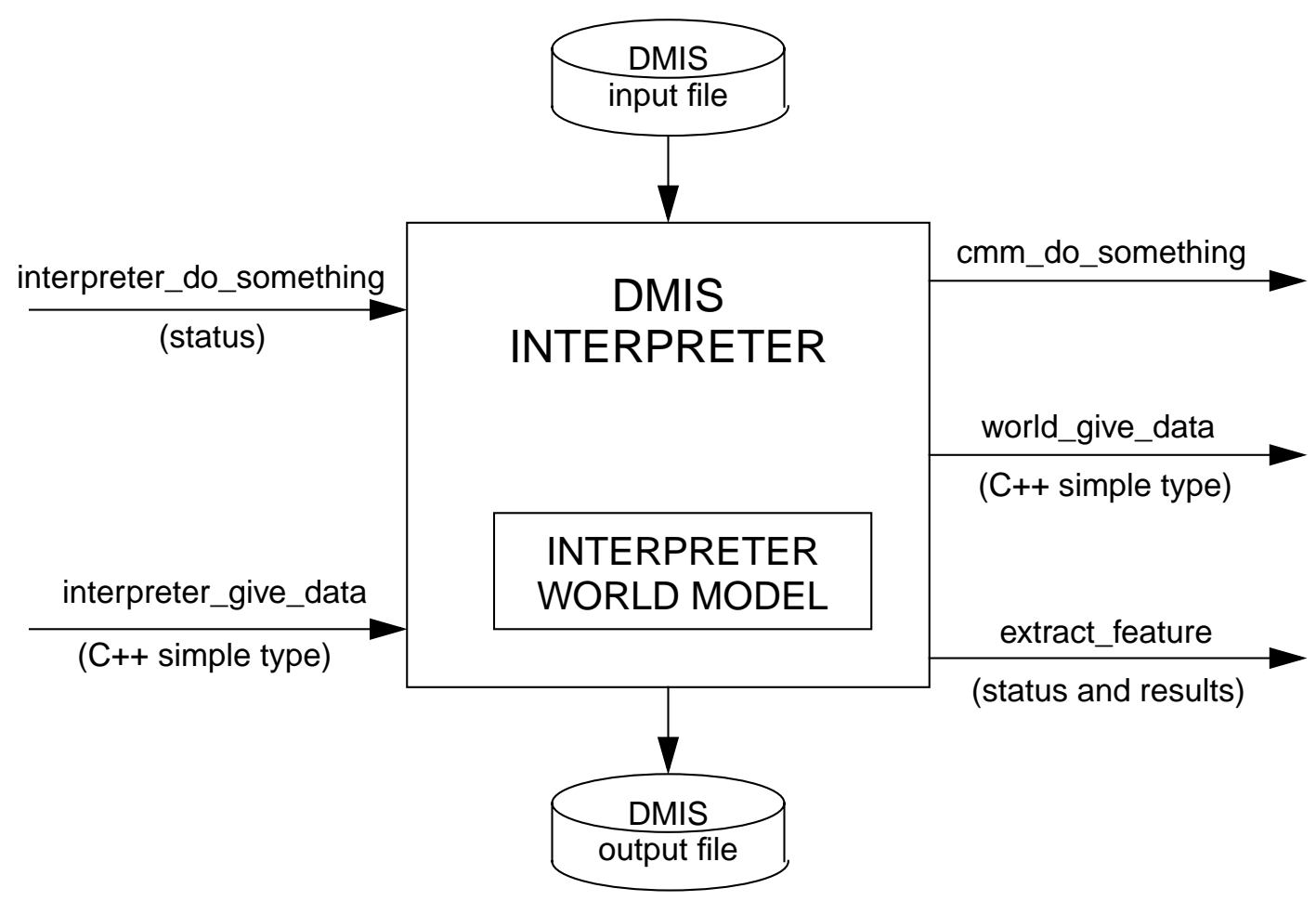

Figure 1. Interpreter Interfaces

The five APIs have been defined in the $\mathrm{C}++$ programming language. For convenience, the 
function prototypes for all five APIs are given in a single header file. For each API there is a separate file that gives the definitions of the functions in the API. In this section we give only the names and arguments of the functions. More details are given in Appendix B, including a description of what each function does.

\subsubsection{Telling the Interpreter What to Do}

The functions in this interface (called interpreter_do_something in Figure 1) are:

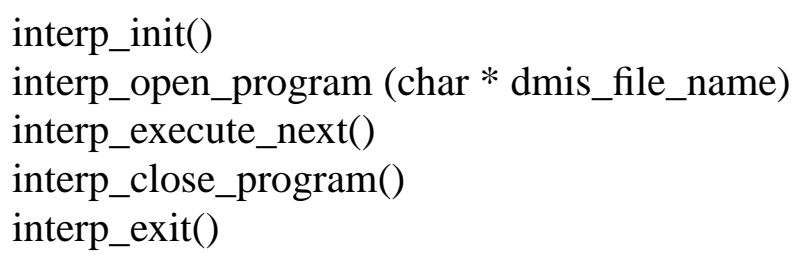

\subsubsection{Getting Data from the Interpreter}

The functions in this interface (called interpreter_give_data in Figure 1) are:

interp_line()

interp_sensor_tip_diameter(char* sensor_name)

\subsubsection{Telling the CMM What to Do}

This interface is called cmm_do_something in Figure 1.

One of the main purposes of the interpreter is to tell the controller what the DMIS program says the equipment should do. To do the telling, a language is needed. A set of "CMM canonical commands" was developed to serve as that language. The CMM canonical commands are listed in Table 1.

The CMM canonical commands are atomic commands. Each command produces a single action.

The correspondence between executing a DMIS statement in the interpreter and the interpreter calling a CMM canonical function is usually one-to-one. Occasionally it is one-to-two. On the other hand, executing many DMIS statements (any statement which is a definition of a feature or tolerance, for example) requires no work on the part of a CMM. In such cases, the "advisory" CMM canonical function is called just to show that the interpreter did something. The "advisory" command contains a message but produces no CMM action. Without the "advisory" command, there would be many cases where executing a DMIS statement would result in no call to any CMM canonical command.

The canonical commands used in the interpreter were devised with three main objectives in mind. First, all the functionality of the existing NGIS had to be covered by the commands; for any function the NGIS can perform, there has to be a way to tell it to do that function. Second, it must be possible to interpret DMIS statements into canonical commands. Third, the canonical commands had to conform to the division of responsibility between the interpreter and the rest of the system, as described in Section 2.4.

Two sets of definitions for the CMM canonical functions have been written, and either set can be linked into the interpreter. The first set is used in the EMC controller for the NGIS testbed. Executing a function from this set causes a command message to be generated. When this command message is executed, the machine's actuators are activated. The second set is used in the stand-alone DMIS interpreter. Executing a function from the second set causes a line of text containing the command to be written to standard output or to a file. 


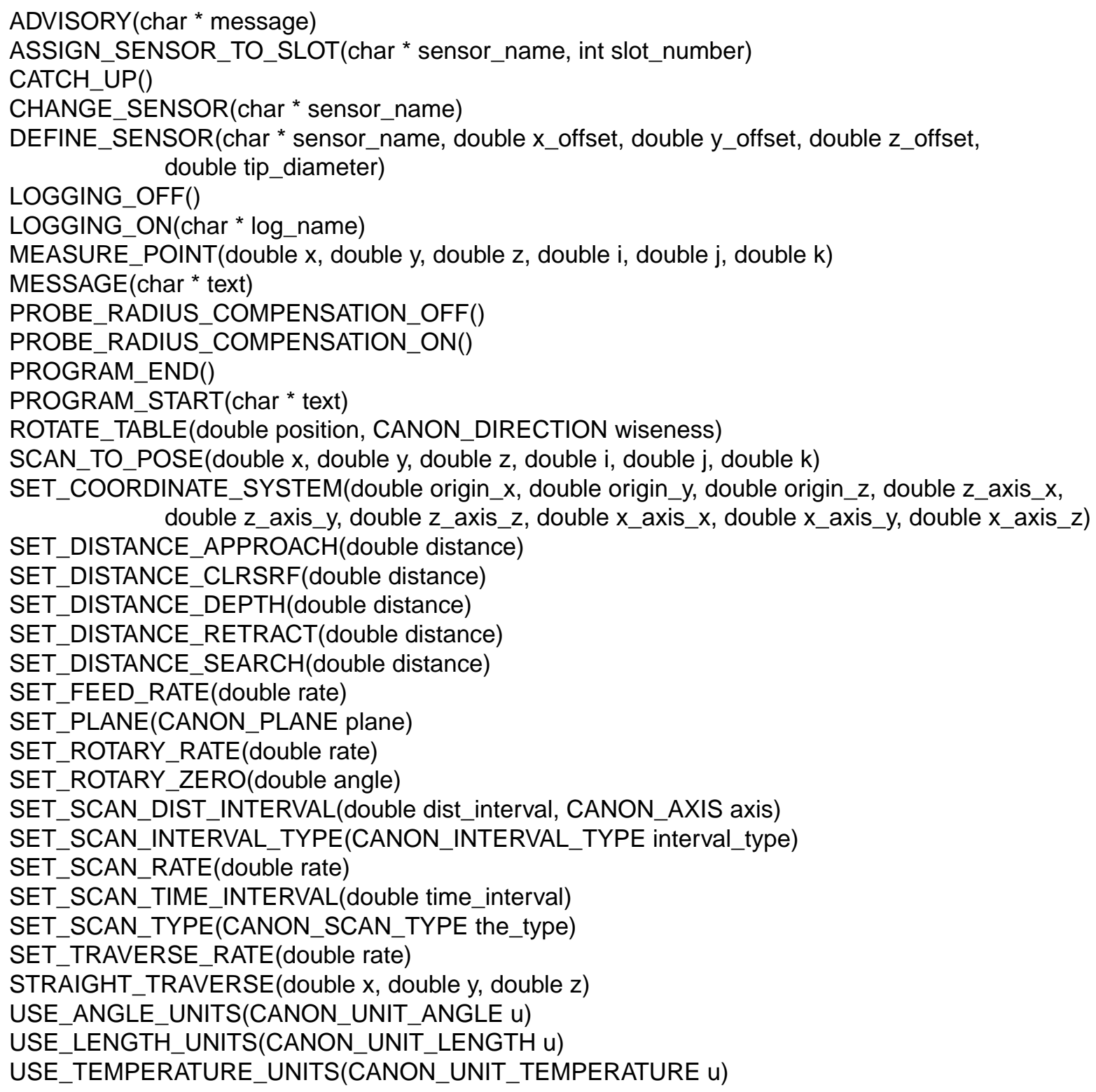

\section{Table 1. CMM Canonical Commands}

Function arguments are written in ANSI C style. All functions return nothing.

\subsubsection{Getting Data from the External World}

This interface is called world_give_data in Figure 1.

The functions in this interface are called by the interpreter. These functions primarily obtain data that is collected during probing. The functions are: 


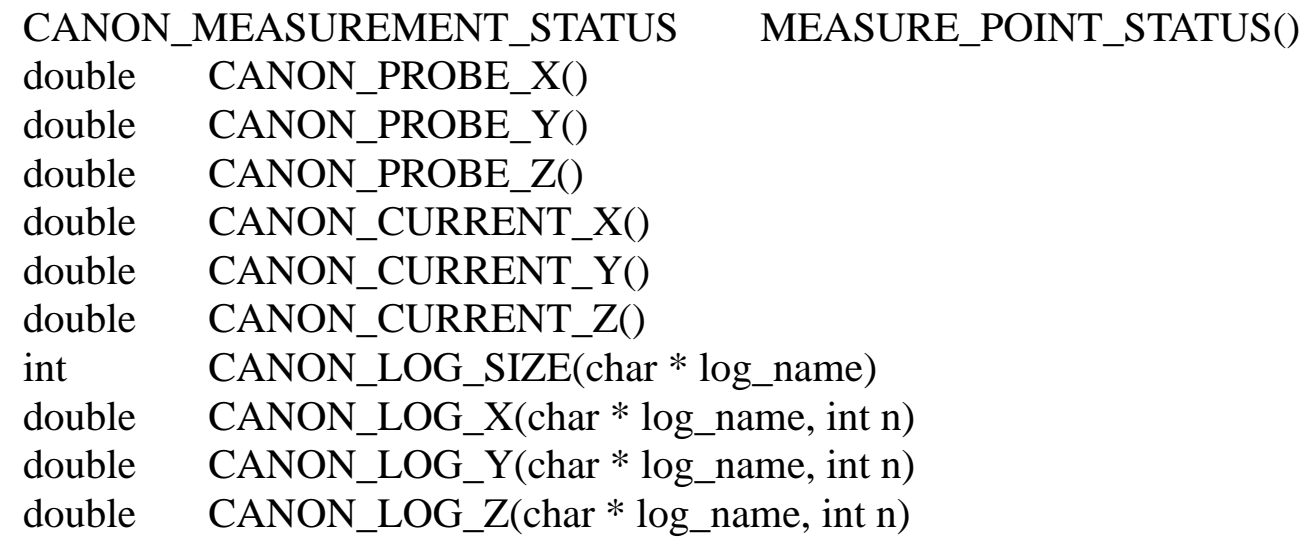

2.1.5 Extracting Feature Parameters from Arrays of Points

Each function in this interface takes an array of points and extracts parameters for a feature from it. The returned value is used only to indicate either OK or error.

The interpreter uses source code for these fitting functions provided by the NIST Algorithm Testing System [Rosenfeld1, Rosenfeld2].

int extract_circle(double points [][3], int how_many, double tolerance, double $*$ center_x, double $*$ center_y, double * center_z, double * normal_i, double * normal_j, double * normal_k, double * diameter)

int extract_cylinder(double points [][3], int how_many, double tolerance, double * center_x, double * center_y, double * center_z, double $*$ direction_i, double $*$ direction_j, double $*$ direction_k, double $*$ diameter)

int extract_line(double points [][3], int how_many, double tolerance, double * point_x, double * point_y, double * point_z, double * direction_x, double * direction_y, double * direction_z)

int extract_plane(double points [][3], int how_many, double tolerance, double * point_x, double * point_y, double * point_z, double * normal_i, double $*$ normal_j, double * normal_k)

int extract_point(double points [][3], int how_many, double tolerance, double * point_x, double * point_y, double * point_z)

\subsection{Integrated or Stand-Alone Operation}

The interpreter runs integrated with the EMC control system or as a stand-alone system. The program interfaces to the interpreter kernel are the same in the two cases. The interfaces seen by a user in the two cases are completely different. The stand-alone system provides a simple textbased command interface for the user; this interface is focused entirely on the interpreter. The EMC control system has a variety of textual and graphic interfaces, only a little of which deals with the interpreter.

In either case, the interpreter first reads the entire DMIS file and stores it as a data structure. Then, the interpreter executes statements one at a time. If there is an error at any point, the interpreter sends a message identifying the nature of the error and stops running. If the error occurs during 
execution, execution stops at the statement where the error occurred, and it is not possible to restart the program from that point. To use a program which causes an interpreter error, the program must be edited to remove the error, and the program must be restarted at the beginning. Further details of error handling are given in Appendix E.1.

In both modes of use, if a DMIS output file is to be written (if there is a FILNAM statement in the input program) the interpreter always writes a DMIS output file named "output.dms".

\subsubsection{Stand-alone}

The stand-alone mode is valuable because it allows a user to pre-test a DMIS program without having to run it on the machine controller. Any computer for which the stand-alone interpreter can be compiled can be used to pre-test DMIS programs. Pre-tests are conclusive tests of whether a program is interpretable or not because the interpreter runs exactly the same way in the standalone mode as it does integrated with the control system. Pre-tests do not show whether the program does what is intended, of course.

The architecture of the stand-alone interpreter is shown in Figure 2. A dummy model of the external world is maintained. The dummy model is changed by the cmm_do_something commands, and the world_give_data commands get data out of the dummy model. Only the cmm_do_something functions which print text have been linked into the stand-alone interpreter, so the output is always text.

The stand-alone interpreter has two modes of use: with or without a command interface.

With the command interface, the user has a finer level of control. It is started by giving the shell command dmis. This brings up a command interface which understands a handful of commands (a list of which is printed if the command help is entered). To interpret a DMIS program line-byline, the user first gives an interp_init() command, then an interp_open_program(input_file_name) command (which causes the entire file to be read and an internal representation built), then a series of interp_execute_next() commands (each of which executes one statement from the program), then an interp_close_program() command. Either an interp_exit() or a quit command will quit the command interface. Also from the command interface, the user may give a run_program(input_file_name) command, which opens, executes, and closes the program.

In the second mode of use, the user gives a single command, in response to which the interpreter reads and interprets an entire DMIS file without bringing up any command interface. This mode is used by giving the shell command dmis input_file_name. In this mode, printed output from cmm_do_something function calls goes to the terminal by default but may be redirected to a file in the normal Unix manner, viz. dmis input_file_name > output_file_name. Even with output redirected this way, the DMIS output file output.dms is still written. 


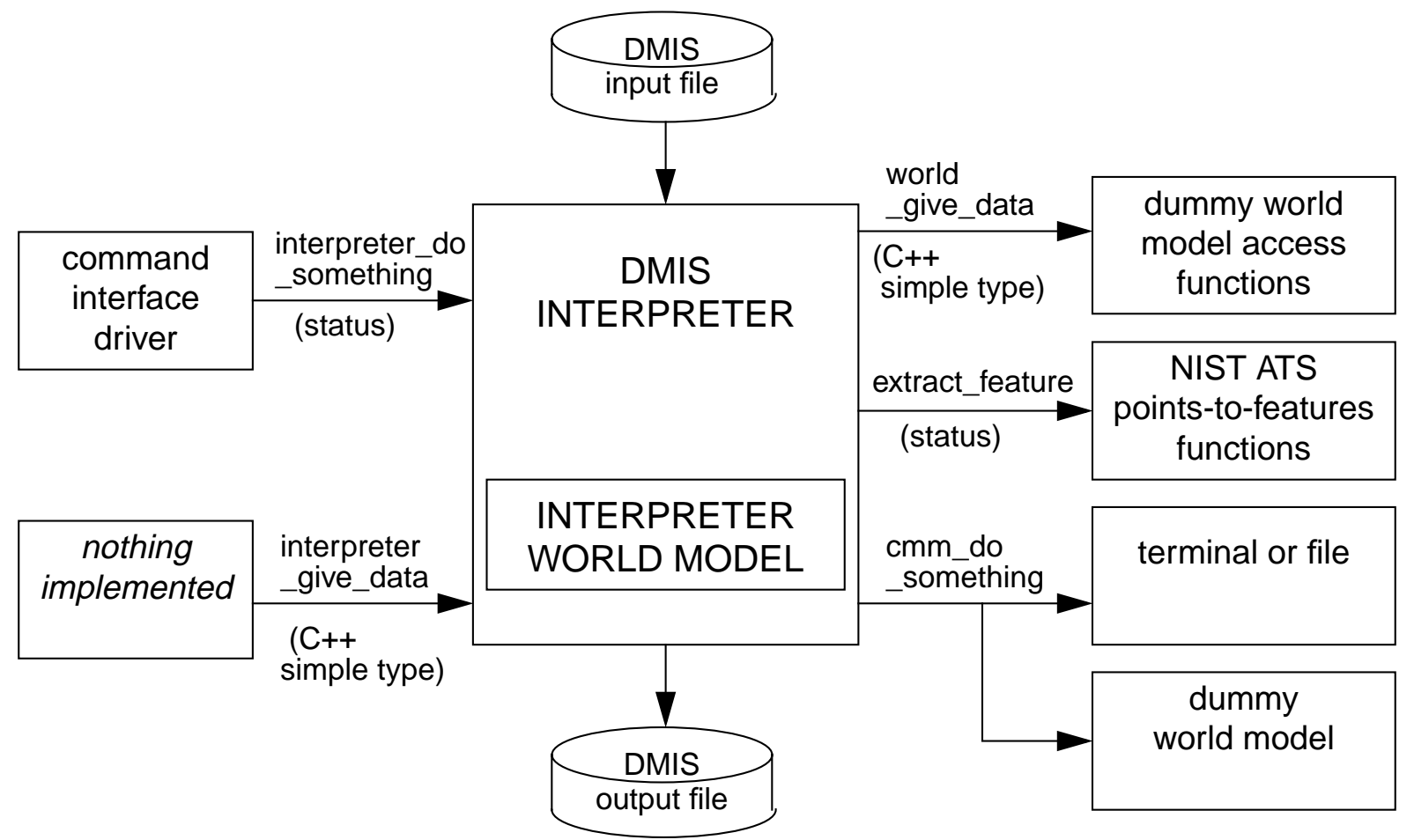

Figure 2. Stand-Alone Interpreter

\subsubsection{Integrated with EMC Control System}

The architecture of the part of the EMC control system that uses the interpreter is shown in Figure 3. The grey box is the EMC controller. The interpreter software is built into the controller.

In this integrated configuration, the control system tells the interpreter when to read the file and when to execute the next statement from the program.

The interpreter does not control machine action directly. Rather, the interpreter calls CMM canonical commands which generate messages that are passed back to the control system, and the control system decides what to do with the messages. 


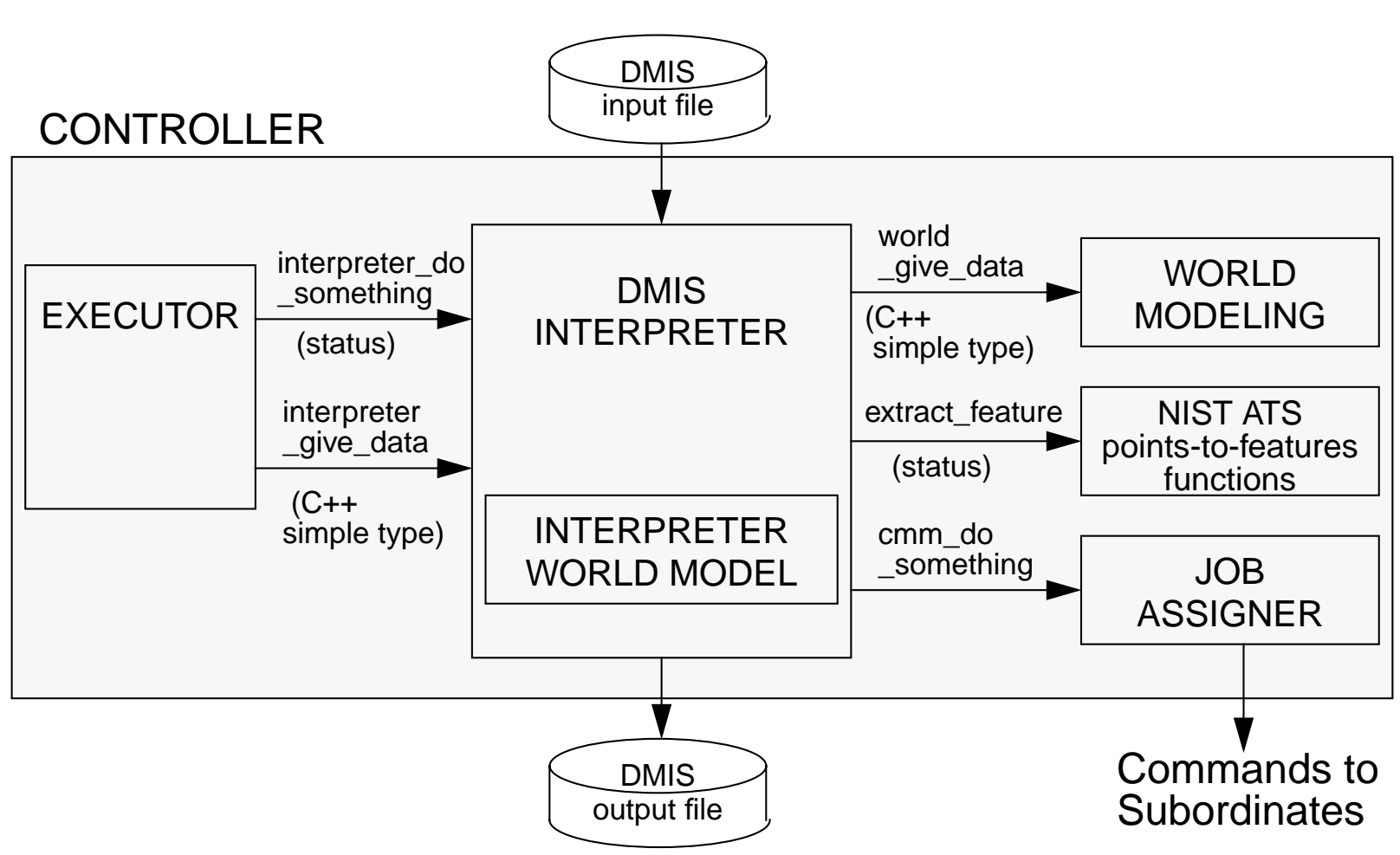

Figure 3. Interpreter Integrated in Controller

\subsection{Major DMIS Interpreter Design Decisions}

The following major design decisions were made regarding the interpreter.

The interpreter software runs in the same process as the executing system. This is to insure that the interpreter can be used conveniently and quickly. With the interpreter tightly integrated this way, communications with the interpreter consist simply of function calls and returned values. Without this tight integration, a more complex method of communicating with the interpreter would have been required. It would be feasible to implement the interfaces to the interpreter using messages sent and received through a communications system.

The executing system controls the interpreter and the CMM; the interpreter does not control the CMM directly. This does not show up in the interfaces to the interpreter. Rather, it is embodied in the definition of the cmm_do_something functions used in the integrated system. These functions just add things to do to the controller's queue. Thus, deciding when to do what is in the hands of the controller's job assigner, where it belongs.

Actions from cmm_do_something functions may be queued, but the interpreter may assume they are executed in order. The interpreter may direct that the queue be emptied before the interpreter is called again. This is so that the interpreter can maintain an accurate model of the world without having to make frequent calls to the world_give_data interface functions.

The executing system handles DMIS input and output via the interpreter. This is to keep the 
burden of dealing with features and tolerances centralized in the interpreter and off the rest of the executing system.

Enough of the DMIS language is implemented to meet the needs of the NGIS project and to handle DMIS programs for two specific parts (the test part for the material removal demonstration of the Department of Energy TEAM program, and the National Aerospace Standard 979 circlediamond-square test part).

The interpreter should be easy to upgrade. In particular, it should be easy to add statements which deal with flow of control.

The interpreter must handle DMIS programs but not single DMIS statements entered by an operator. The capability to handle single statements could be added, if needed.

\subsection{Division of Responsibilities}

The DMIS interpreter is part of the executing system. This section discusses how responsibilities are divided between the DMIS interpreter and the rest of the executing system.

\subsubsection{Control}

The rest of the executing system performs control. The interpreter controls nothing; it only advises the rest of the system what the DMIS program says to do.

\subsubsection{Languages}

The interpreter understands DMIS statements and can interpret them to produce cmm_canonical commands. The rest of the executing system does not understand DMIS statements but can carry out cmm_canonical commands.

\subsubsection{DMIS output}

The interpreter produces DMIS output as required by DMIS program. The rest of the executing system does not deal with DMIS output in any way.

\subsubsection{Coordinate systems}

The interpreter remembers all coordinate systems in a DMIS program. The rest of the executing system deals with one active coordinate system, which may be changed. One coordinate system both the interpreter and the rest of the system understand is the machine coordinate system. Each change of coordinate system is expressed to the rest of the system in terms of the machine coordinate system.

\subsubsection{Features and Tolerances}

The interpreter remembers all nominal DMIS features (and corresponding actual features if and when actual features are created). The interpreter handles all DMIS tolerances.

The rest of the executing system does not have the concept of a DMIS feature or a tolerance.

\subsubsection{Units}

The interpreter and the rest of the executing system both understand length $(\mathrm{cm}$, inch, feet, $\mathrm{m}$, $\mathrm{mm}$ ), angle (decimal degrees, radians), and temperature (centigrade, Fahrenheit) units and can change them. It might be simpler to let the executing system deal with only one unit of each type; this would certainly be feasible. 


\subsubsection{Sensors}

The interpreter remembers all sensor definitions in a DMIS program, but data required by the rest of the executing system might not be in DMIS program. The rest of the system remembers sensors by name and can remember the diameter of a sensor tip. By using an interpreter_give_data command, the rest of the system can ask the interpreter for the tip diameter of a sensor by name. The rest of the executing system can change sensors.

\subsection{How the Interpreter Runs}

To interpret a program, the interpreter is given an interp_init command, followed by an interp_open_program command, followed by many interp_execute_next commands.

The interpreter maintains a model of the machine while it interprets and uses the model in determining what cmm_canonical functions to call and what their arguments should be. The model is initialized when the interpreter is started by a call to the interp_init command.

When the interp_open_program command is given, the interpreter reads an entire DMIS program into active memory before any of the DMIS statements in it are interpreted. Then DMIS statements are interpreted one at a time.

The interpreter uses two intermediate files to help get statements from a DMIS program file into active memory. The format of the second intermediate file and the structure of the representation in active memory use techniques from STEP and STEP utilities, as discussed in Appendix A and Appendix F.

In carrying out an interp_open_program command, the interpreter does the following:

1. The entire original DMIS program is read. It is stripped of comments and continued lines are joined. The DMIS file "dmis_to_step_temp" is written.

2. The file "dmis_to_step_temp" is read and a STEP file "dmis_to_step.step" is written.

3. The file "dmis_to_step.step" is read, creating a large in-core structure, usable by the interpreter, that represents the entire DMIS program. This structure is made up mainly of substructures representing DMIS statements.

Calling interp_execute_next causes the interpreter to interpret the statement which should be executed next. The statement that should be executed next is not necessarily the one on the line after the last line that was executed.

The structure of a DMIS program is, in general, a nested hierarchy of blocks of statements. The interpreter maintains a stack which mirrors the program structure. The stack is used to help decide which statement is the next one that should be executed and to remember important data about each block.

After a program has reached the last command to be executed (or in the middle of a program, if that is desired), an interp_close_program command should be given. This returns the interpreter to the state it was in before the program was opened.

To stop the interpreter entirely, an interp_exit command should be given. This should not be done when a program is open. 
NIST DMIS Interpreter 


\begin{tabular}{|c|c|}
\hline Item & Meaning \\
\hline double angle_factor & factor to multiply for radians \\
\hline angle_unit_type angle_units & current angle units \\
\hline datum_definition * current_system & current coordinate system \\
\hline double current_position[3] & current position $\mathrm{x}, \mathrm{y}, \mathrm{z}$ \\
\hline double current_table & current rotary table position \\
\hline double defalt_feed & default linear feed rate \\
\hline double defalt_rotate & default rotary table rate \\
\hline double defalt_scan & default scan feed rate \\
\hline double defalt_traverse & default linear traverse rate \\
\hline datum_definition $*$ default_system & default machine coord system \\
\hline char error_message[TEXT_SIZE] & latest error message \\
\hline int first_line & flag that first line found \\
\hline double high_feed & high linear feed rate \\
\hline double high_rotate & high rotary table rate \\
\hline double high_scan & high scan feed rate \\
\hline double high_traverse & ligh linear traverse rate \\
\hline ListOfListOfdmis_item item_stack & control structure \\
\hline double length_factor & to convert to millimeters \\
\hline line * exec_line & currently executing line \\
\hline double low_feed & low linear feed rate \\
\hline double low_rotate & low rotary table rate \\
\hline double low_scan & low scan feed rate \\
\hline double low_traverse & low linear traverse rate \\
\hline double max_feed & maximum linear feed rate \\
\hline double max_rotate & maximum rotary table rate \\
\hline double max_scan & maximum scan feed rate \\
\hline double max_traverse & maximum linear traverse rate \\
\hline on_off_type mode_auto & whether in AUTO mode \\
\hline on_off_type mode_man & whether in MAN mode \\
\hline on_off_type mode_prog & whether in PROG mode \\
\hline on_off_type output_dmis & whether to output DMIS \\
\hline FILE $*$ output_file & output FILE pointer \\
\hline char output_file_name[TEXT_SIZE] & DMIS output file name \\
\hline double points[1000][3] & array to hold point data \\
\hline int point_number & number of points in array \\
\hline on_off_type point_stuff_flag & whether to put point in array \\
\hline double position_tolerance & positioning tolerance \\
\hline double probe_x & last probed location $\mathrm{x}$ \\
\hline double probe_y & last probed location y \\
\hline double probe_z & last probed location $\mathrm{z}$ \\
\hline char program_file_name[TEXT_SIZE] & program file name \\
\hline RoseDesign * program_pointer & program structure \\
\hline on_off_type & whether to scan \\
\hline on_off_type update_flag & whether to update positions \\
\hline
\end{tabular}

Table 2. Interpreter Internal Model 


\subsection{Interpreter Model}

The interpreter maintains one global variable, “_interp". This is an instance of the "interp" class, whose attributes are shown in Table 2. All data required by the interpreter (including the current DMIS program, for example) is incorporated in the _interp model.

\subsection{Speed}

The stand-alone interpreter, running on a SUN SPARCstation 20, read and executed a 720-line program in about 5 seconds. Just reading the same file (not executing anything) took about 2 seconds. Carrying out that program on a CMM using the integrated controller would take several minutes. Shorter programs take so little time for the stand-alone interpreter that it is hard to measure. We have not identified any situation in which the speed of the interpreter causes any problem in the rest of the system.

The only interpreter operation which we believe could take a significant amount of time is fitting features to sets of measured points. In a few tests, a fitting function ran for up to 30 seconds. That is the slowest we have encountered.

\subsection{Limitations of the Interpreter}

The interpreter implements the parts of DMIS which are expected to be most heavily used, but this includes only about a quarter of the language. Several fairly common feature types, including sphere and cone, are not implemented. No statements for transfer of control, such as IF-ELSE, DO, or CASE are implemented. Expressions are not implemented.

The currently used functions that extract feature parameters from sets of points, which we obtained from another NIST division, do not appear to be reliable. The interpreter should be used with great caution until this problem is resolved, since, in many DMIS programs, the parameters of a feature may be used to determine subsequent motion. New versions of these functions have been received but not yet incorporated in the interpreter and tested.

As mentioned earlier, the interpreter requires an entire program as input. The interpreter would need to be modified to handle single DMIS statements, a desirable capability for direct control by an operator.

\section{Input}

\subsection{Overview}

In general, allowable inputs are as described in [CAM-I] and discussed earlier in Section 1.2.

\subsubsection{Case, White Space, Line Continuations, Comments.}

The DMIS language is case insensitive [CAM-I, page 12]. Any letter may be in upper or lower case without changing the meaning of a statement, except that, within text strings, case is preserved. The interpreter implements these rules.

Blank lines are allowed in DMIS and in the input by the interpreter [CAM-I, page 11]. They are ignored. White space (spaces or tabs) is allowed between DMIS words, but not within them. 
White space is preserved within text strings, however.

A single line may have a maximum length of 80 characters, but statements may consist of several lines by putting a ' $\$$ ' sign as the last printing character on each line to be continued. The maximum length of a statement allowed in [CAM-I, page 11] is 256 characters, but the interpreter will handle much longer statements. Up to 420 characters has been tested.

Lines are terminated with a carriage return (ASCII 13 (base ten)) and line feed (ASCII 10 (base ten)).

A line starting with ' $\$ \$$ ' is a comment and is ignored by the interpreter [CAM-I, page 11]. The double dollar sign is not allowed elsewhere. In the interpreter, it will cause an error during parsing if used anywhere except at the beginning of the line.

\subsection{Input Statements}

The formal specification of an allowable program is defined in Appendix G. The description here is intended to be consistent with the appendix. In order that the definition in the appendix not be unwieldy, some constraints imposed by the interpreter are omitted from that appendix. The list of error messages in Appendix E indicates all of the additional constraints.

\subsubsection{Format of a DMIS Statement}

DMIS statements are of two main types: definitions and not-definitions.

Definitions are any of the following (not-definitions are everything else):

coordinate system

feature

label assignment

rotary table

sensor

tolerance

Except for label assignment, a definition statement uses the form thing defined $=$ definition. For example, a cylinder named CYL_A may be defined as follows.

$\mathrm{F}\left(\mathrm{CYL} \_\mathrm{A}\right)=\mathrm{FEAT} / \mathrm{CYLNDR}, \mathrm{INNER}, \mathrm{CART}, 0.0,0.0,0.0,0.0,0.0,1.0,5.0$

A DMIS statement may be a single DMIS major word on a line, such as:

ENDMES

More commonly, a statement is a DMIS major word followed by a slash and modifiers. Modifiers may be DMIS minor words, text strings, numbers, or labels of defined things. Here are a few examples of statements.

FILNAM/'teampart inspection results from demol.dms'

FEDRAT/SCNVEL, MPM, 0.6

MEAS/POINT, F(START_PT), 1

PTMEAS/CART, 5.0, $0.0,-60.0,0.0,1.0,0.0$

\subsubsection{Numbers}

The manual [CAM-I, sec. 2.1.3, p. 5] is unclear in defining a valid number. In the formal specification used by the interpreter, given in Appendix G, the following rules regarding numbers 
may be found. In these rules a digit is a single character between 0 and 9 .

A "LIL_INTEGER" is one or two digits separated from other characters. A "BIG_INTEGER" is three or more digits separated from other characters. An "integer" is either a LIL_INTEGER or a BIG_INTEGER.

A "REAL" is a sequence of characters that does not qualify as an integer but consists of (i) an optional plus or minus sign, followed by (ii) zero to many digits, followed, possibly, by (iii) one decimal point, followed by (iv) zero to many digits - provided that there is at least one digit somewhere in the number.

A "real" is a "REAL"1 or an "integer". Character strings that form numbers are interpreted in the usual way. For example, a non-zero number with no sign as the first character is assumed to be positive.

With these definitions, the following observations are implicit.

Initial (before the decimal point and the first non-zero digit) and trailing (after the decimal point and the last non-zero digit) zeros are allowed but not required. A number written with initial or trailing zeros will have the same value when it is read as if the extra zeros were not there.

Numbers may have any number of digits, subject to the limitation on line length.

Exponential notation is not allowed.

\subsubsection{Line Number}

The DMIS language does not provide for line or statement numbers. The interpreter keeps track of statement numbers by counting them. The first statement is assigned number 1 , the second 2 , and so on.

\subsection{Words Recognized}

The interpreter recognizes statements beginning with the 46 major word / minor word combinations shown in Table 3. These are all listed in the Index of Statements in [CAM-I, p. 389]. The meanings of the statements are as given in [CAM-I]. In most cases, not all of the possible variants of a statement are implemented. Any capability not explicitly included is excluded. Trying to use any excluded capability in a DMIS program will cause an error in the interpreter.

1. The formal specification is case sensitive, so "real" differs from "REAL". 
NIST DMIS Interpreter 


\begin{tabular}{|c|c|}
\hline Code & Meaning \\
\hline CONST Format 1 & construct a feature \\
\hline DATDEF & assign a datum label \\
\hline DATSET & define a coordinate system \\
\hline DISPLY & specify output devices and formats \\
\hline DMISMN & begin a DMIS program \\
\hline ENDFIL & end a DMIS program \\
\hline ENDGO & end a GOTARG block \\
\hline ENDMES & end a MEAS block \\
\hline FEAT/CIRCLE & define a circle \\
\hline FEAT/CYLNDR & define a cylinder \\
\hline FEAT/LINE & define a straight line \\
\hline FEAT/PLANE & define a plane \\
\hline FEAT/POINT & define a point \\
\hline FEDRAT & set a feedrate \\
\hline FILNAM & assign an output file name \\
\hline GOTARG & begin a block of free space moves \\
\hline GOTO & make a free space move \\
\hline MEAS & begin a block which measures a feature \\
\hline MODE & select program modes \\
\hline OUTPUT & output data on features or tolerances \\
\hline PRCOMP & turn probe tip diameter compensation on or off \\
\hline PTMEAS & measure a point \\
\hline RECALL & reactivate a coordinate system, sensor, or feature \\
\hline ROTAB & rotate a rotary table \\
\hline ROTATE & rotate a coordinate system \\
\hline ROTDEF & define a rotary table \\
\hline ROTSET & reset the angle of a rotary table \\
\hline SAVE & save coordinate systems, features, etc. for later recall \\
\hline SCNMOD & turn scanning mode on or off \\
\hline SCNSET & specify how scanning will be done \\
\hline SNSDEF Format1 & define a sensor \\
\hline SNSET & specify probing parameters \\
\hline SNSLCT & change sensors \\
\hline TEXT & send text to the operator or output file \\
\hline THLDEF & define a tool holder \\
\hline TOL/CORTOL & define a coordinate tolerance \\
\hline TOL/CYLCTY & define a cylindricity tolerance \\
\hline TOL/DIAM & define a diameter tolerance \\
\hline TOL/FLAT & define a flatness tolerance \\
\hline TOL/PARLEL & define a parallelism tolerance \\
\hline TOL/PERP & define a perpendicularity tolerance \\
\hline TOL/POS & define a position tolerance \\
\hline TRANS & translate a coordinate system \\
\hline UNITS & specify units \\
\hline VFORM & specify output in vendor format \\
\hline WKPLAN & select a working plane \\
\hline \multicolumn{2}{|c|}{ Table 3. DMIS Words Implemented in the Interpreter } \\
\hline
\end{tabular}




\section{References}

[Albus] Albus, J.S.; Meystel, A. M.; A Reference Architecture for Design and Implementation of Intelligent Control in Large Complex Systems; International Journal of Intelligent Control and Systems; Vol. 1, No. 1; 1996; 15-30

[CAM-I] Consortium for Advanced Manufacturing - International; Dimensional Measuring Interface Standard; Revision 3.0, ANSI/CAM-I 101-1995; CAM-I, Arlington, Texas; 1995

[ISO1] ISO 10303-11:1994; Industrial automation systems and integration - Product data representation and exchange - Part 11: The EXPRESS Language Reference Manual; ISO; Geneva, Switzerland; 1994

[ISO2] ISO 10303-21:1994; Industrial automation systems and integration - Product data representation and exchange - Part 21: Clear Text Encoding of the Exchange Structure; ISO; Geneva, Switzerland; 1994

[Kramer] Kramer, Thomas R.; Proctor, Frederick M.; The NIST RS274/VGER Interpreter; NISTIR 5754; National Institute of Standards and Technology; Gaithersburg, MD; 1995

[Levine] Levine, John; Mason, Tony; Brown, Doug; lex \& yacc; 2nd Edition; O’Reilly \& Associates; Sebastopol, CA; 1992

[Loffredo1] Loffredo, David; et al; STEP Utilities Reference Manual; STEPTools Inc.; Troy, NY; 1994

[Loffredo2] Loffredo, David; et al; ROSE Library Reference Manual; STEPTools Inc.; Troy, NY; 1993

[Proctor] Proctor, Frederick M.; et al; Simulation and Implementation of an Open Architecture Controller; Proceedings of the SPIE International Symposium on Intelligent Systems and Advanced Manufacturing; Philadelphia, PA; 1995

[Rosenfeld1] Rosenfeld, David A.; User's Guide for the Algorithm Testing System Version 2.0; NISTIR 5674; National Institute of Standards and Technology; Gaithersburg, MD; 1995

[Rosenfeld2] Rosenfeld, David A.; Reference Manual for the Algorithm Testing System Version 2.0; NISTIR 5722; National Institute of Standards and Technology; Gaithersburg, MD; 1995 


\section{Appendix A Software Details}

This appendix describes the software for the interpreter. The appendix is intended for users and programmers who want to modify the software or simply to understand it.

\section{A.1 Overall Approach}

The interpreter is written in $\mathrm{C}++$. Four additional languages were used: lex [Levine], YACC [Levine], EXPRESS [ISO1], and STEP Part 21 [ISO2]. Tools for dealing with these languages were used to simplify the work, as described below.

\section{A.1.1 YACC and lex}

DMIS is a large language with hundreds of allowed formats for statements. Thus, it was clear that building a parser from scratch directly in $\mathrm{C}++$ would be very time-consuming. YACC (Yet Another Compiler Compiler) grammar is a widely available, widely used language for specifying valid input for parsers. The DMIS manual is written in a style quite similar to that used for specifying a grammar in YACC. YACC, therefore, was chosen as the language for input parsing. Processing input specified in a YACC grammar requires a lexical scanner. The language for describing lexical input normally used with YACC is lex, so that was an obvious choice. The YACC grammar used in the interpreter is shown (without actions) in Appendix G.

DMIS input may include comments and line continuations. It is difficult to write a YACC grammar that deals with these items wherever they might appear, but it is rather easy to remove them as a pre-process. A brief lex specification was written for this purpose. Thus, the interpreter uses lex twice.

A file of $\mathrm{C}$ code for a lexical scanner and a file of $\mathrm{C}$ code for a parser are generated automatically by utilities which read the specifications and write the code. The usual utilities for handling lex and YACC files have the same names as the languages. However, there are other utilities for handling such files available from the Free Software Foundation, namely "flex" (fast lex) and "bison" (a mammal similar in appearance to a yak). These are somewhat superior to the original lex and yacc processors available to us and were used in building the interpreter.

\section{A.1.2 Read All First}

For several reasons, it was decided to read and store the entire DMIS program before executing any of it. First, it saves time during execution, which may be important because of motion control requirements. Second, it prevents getting part way through the execution of a program and then discovering it has an error. This often requires fixing the error and rerunning the entire program. Third, it is convenient to program. Input may be handled in one module and interpretation in another, rather than having to interleave them.

\section{A.1.3 EXPRESS}

EXPRESS is a data modeling language used in ISO standard 10303, commonly called STEP. The EXPRESS language [ISO1] allows the definition of types of data either as TYPES (similar to types in $\mathrm{C}$ or $\mathrm{C}++$ ) or as ENTITIES (similar to structs in $\mathrm{C}$ or $\mathrm{C}++$ ). As compared with $\mathrm{C}$ or $\mathrm{C}++$, EXPRESS is richer in providing for constraints on data and making it easy to specify a range of choices for a data type. EXPRESS, however, does not provide methods and is not compilable into 
an executable computer program. The most inclusive construct in EXPRESS is the "schema", a collection of interrelated definitions of TYPES, ENTITIES, and other EXPRESS constructs. A schema generally serves (alone or with related schemas) as a model of something.

Storing a representation of a DMIS program (like storing anything else in an executing system) requires a data structure with accompanying access functions to put data into it originally, modify data, and get data out. If this were done directly in $\mathrm{C}++$, header files would need to be designed and written and $\mathrm{C}++$ access functions would need to be written. There is a utility available from STEPTools, Inc. (and there are several similar utilities available from other sources) called express2c++ [Loffredo1]. This utility takes an EXPRESS schema as input and produces $\mathrm{C}++$ code as output. The code includes both header files and access functions. The design of the header files is inherited from the design of the EXPRESS schema. In addition to providing express $2 \mathrm{c}++$, STEPTools provides a library of functions which work with the automatically generated $\mathrm{C}++$ code [Loffredo2]. The library includes utilities for reading and writing files (in STEP Part 21 exchange file format [ISO2]) and for performing additional operations on the in-core model produced by reading a file, beyond what is available using the access functions generated by express2c++. Thus, if there is an EXPRESS model available, almost all the work of designing a data structure and implementing the design in $\mathrm{C}++$ can be eliminated.

This method was used with the DMIS interpreter and resulted in a net saving of four weeks of programming time (best guess). The system builder was already very familiar with EXPRESS, Part 21 exchange files, and the STEPTools utilities. Using this method would not have saved time if much learning had been required - although the learning only needs to be done once per person and is reusable. STEPTools version 1.4 was used in the interpreter.

Using STEPTools has two disadvantages. First, the STEPTools library is required, so the system can only be ported to systems on which that library is available. Second ${ }^{1}$, the code automatically generated by express2c++ is very extensive and takes up a lot of memory space when compiled. Much of this code is unused and could be eliminated, but determining what is unused and eliminating it would be time-consuming and would need to be repeated each time the EXPRESS schema was modified.

The EXPRESS schema for the part of DMIS that has been implemented in the interpreter is shown in Appendix F and discussed further there.

\section{A.1.4 YACC and EXPRESS}

Using EXPRESS and YACC together was accomplished by having the YACC parser write a Part 21 exchange file, which is always named dmis_to_step.step, and then using the STEPTools file reader to read dmis_to_step.step. We believe the actions in the YACC grammar are simpler for writing a Part 21 file than they would have been if they had been constructing an in-core representation of the DMIS program directly, which is what they would have done if EXPRESS had not been used.

\section{A.2 Software Modules}

Two methods of using the interpreter, stand-alone and integrated with the rest of EMC, are

1. It is possible that the second problem has been alleviated in version 1.5 of STEPTools, which is the current release. 
provided, as described in Section 2. The use of program files differs between the two methods. Some code is common to both, and some code differs.

\section{A.2.1 Stand-Alone and Integrated}

The program files used for both the stand-alone and integrated interpreter are:

C++ Header Files

1. cmm_canon.hh - 1,492 lines (mostly text)

This is for the interpreter's five interfaces.

2. dmis_schema.h - 5,099 lines

This is for the data structures and access functions needed for dealing with the EXPRESS schema for DMIS. It was generated automatically by express $2 \mathrm{c}++$.

3. fit.hh - 341 lines

This is for the ATS feature fitting functions and was written by ATS personnel.

4. interp.hh - 121 lines

This is for the interpreter kernel. The bulk of it gives the structure of the internal interpreter world model.

5. tk_common.hh - 35 lines

This is a small collection of constants and macros to simplify programming.

$\mathrm{C}++$ Function Definitions

1. cmm_canon_data_out.cc - 36 lines

This is for the interpreter_give_data interface to the interpreter.

2. cmm_canon_extract1.cc - 5,673 lines

This is the extract_feature functions. All but 511 lines of this was written by ATS personnel.

3. cmm_canon_interp.cc - 208 lines

This is for the interpreter_do_something interface to the interpreter.

4. dmis_schema.cc - 4,502 lines

This is the access functions for dealing with DMIS schema data. It was generated automatically by express $2 \mathrm{c}++$.

5. interp.cc - 14,528 lines

This is the interpreter kernel. About half of this was generated automatically by flex and bison from lex and YACC source, and half hand-written. The lex source totals 484 lines, while the YACC source is 1,784 lines.

\section{A.2.2 Stand-Alone Only}

The program files used in the stand-alone interpreter only are:

C++ Header Files

1. rest_world.hh - 48 lines

This defines the structure of the dummy world model used by the stand-alone interpreter.

C++ Function Definitions

1. cmm_canon_do_it.cc - 417 lines

This is for the interpreter's cmm_do_something interface. The functions defined in this file 
print themselves. Some of them also alter the dummy world model to simulate being executed.

2. cmm_canon_data_in.cc - 152 lines

This is for the interpreter's world_give_data interface. The functions extract data from the dummy world model.

3. driver.cc - 281 lines

This provides an interface to the user.

\section{A.2.3 Integrated Only}

The interpreter program files used only in the integrated interpreter follow. In addition to these specific files, other files which are not considered to be part of the interpreter provide the world model actually used by the rest of the controller in which the interpreter lies.

C++ Function Definitions

1. cmm_canon_do_it.cc

This is for the interpreter's cmm_do_something interface. The functions defined in this file mostly generate command messages.

2. cmm_canon_data_in.cc

This is for the interpreter's world_give_data interface. The functions extract data from the actual world model.

\section{A.3 Source Code Documentation}

The source code is heavily documented. In general, for each function, four fields are given:

1. Returned Value - a description of possible returned values and the circumstances in which particular values may be returned. In most kernel functions, either OK or ERROR may be returned.

2. Side Effects - a description of the important side effects (things other than the returned value) of executing a function. Since the returned value of most functions is used to indicate error status, the side effects of most functions are important.

3. Called By - a list of functions which call the function being documented.

4. Argument Values - a one-line description of the meaning of each argument to a function, placed immediately after the declaration of the argument. This field is omitted if there are no arguments.

In addition to these four fields, most functions have a paragraph to a page of discussion. Where a function implements an algorithm for geometric or numerical calculation, the algorithm is described. Many citations to specific pages of the CAM-I manual are included in these discussions. 


\section{Appendix B Interpreter Interface Functions}

As described in Section 2, the interface between a DMIS interpreter and a software system in which the interpreter is working comprises five sets of commands. The commands are represented here as $\mathrm{C}$ (or $\mathrm{C}++$, which is identical for these usages) function definitions. The five sets are:

1. Functions which extract data from the interpreter.

2. Functions for the interpreter to call which extract data from the rest of the system.

3. Functions for the rest of the system to call to tell the interpreter to do something. These are normally called by a controller for which the interpreter works.

4. Functions for the interpreter to call which tell the rest of the system to do something which has been specified by a DMIS program or which the interpreter needs the rest of the system to do.

5. Functions for deriving "actual" feature parameters from point sets. For example, finding the center, plane, and radius of a circle, given three points in 3D space.

For some of these sets of functions, data type definitions are required. The definitions are given here as typedef's at the beginning of each section.

\section{B.1 Functions Which Extract Data From the Interpreter}

Functions which extract data from the interpreter are intended to be called by the system using the interpreter.

All the function names in this set start with "INTERP"

\section{int INTERP_LINE ()}

This returns the line number of the line from the file dmis_to_step_temp (see Section 2.5) which is currently being executed by the interpreter. If no line is currently being executed, this returns the number of the line last executed. In the _interp model, the line number is updated when execution of a line starts.

\section{double INTERP_SENSOR_TIP_DIAMETER (char * sensor_name)}

This returns the diameter of the tip of the named sensor in current length units. If the interpreter has no record of a sensor of the given name, -1.0 is returned.

This would be used if the executing system does not know the diameter of the sensor tip and needs it to do probe tip radius compensation.

\section{B.2 Functions for the Interpreter to Call to Get World Model Data}

This set of functions is intended to be used by the interpreter. The value of data about the world outside the interpreter is expected to be returned in most cases.

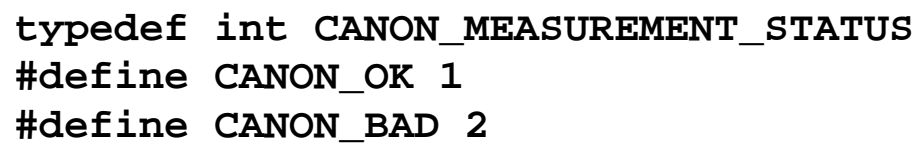

CANON_MEASUREMENT_STATUS is used as a return value of the measure_point_status 
function.

CANON_MEASUREMENT_STATUS MEASURE_POINT_STATUS ( )

MEASURE_POINT_STATUS returns the status of the last MEASURE_POINT action. OK means the last measurement worked successfully. BAD means it did not. Typically, a measurement will be bad if the probe did not trip, or it tripped before it should have, or it was not on, or it appeared to work but returned an out of range value, etc.

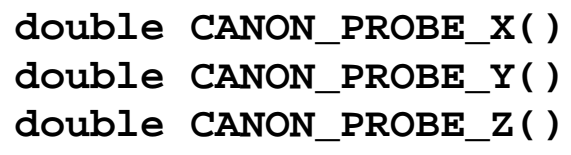

CANON_PROBE_X returns the last recorded probe $\mathrm{X}$-value (in the current coordinate system) resulting from a MEASURE_POINT function call. If probe compensation is on, this is the system's best estimate of the X-coordinate of the probed point. Otherwise, this is the system's best estimate of the $\mathrm{x}$-coordinate of the location of the controlled point (normally the probe tip) when the point was contacted. CANON_PROBE_Y() and CANON_PROBE_Z() behave similarly.

\section{double CANON_CURRENT_X() \\ double CANON_CURRENT_Y() \\ double CANON_CURRENT_Z()}

CANON_CURRENT_X returns the current $\mathrm{X}$-value of the controlled point (in the current coordinate system). CANON_CURRENT_Y() and CANON_CURRENT_Z() behave similarly.

\section{int CANON_LOG_SIZE (char * log_name)}

CANON_LOG_SIZE returns the number of points in the named log, if it exists. If the log does not exist, -1 should be returned.

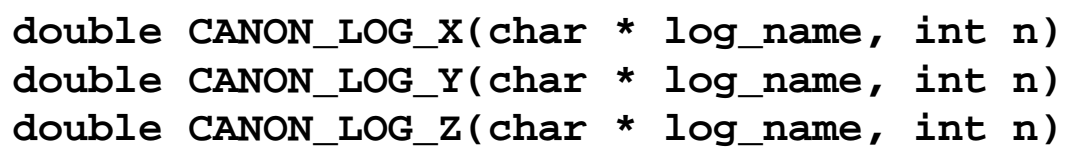

CANON_LOG_X returns the $x$-value of the nth point from the log of the given log_name. For the first point, $\mathrm{n}$ is 1 . If this function is called and the $\log$ does not exist or $\mathrm{n}$ is larger than the number of points in the log, zero should be returned (changing this to return something more useful than zero would be a good idea, since zero could be a reasonable X-value). CANON_LOG_Y() and CANON_LOG_Z() behave similarly.

\section{B.3 Functions to Tell the Interpreter What to Do}

Conceptually, the interpreter has four states:

1. Down 


\section{Ready}

3. Working (on a program)

4. Finished (working on a program)

These states are implicit and not explicit in the software.

Legal calls to functions in the interp-do-something interface, when in specific states, are shown in Table 4. The state in effect after each call (if the call does not return ERROR) is also shown in the table. Any call which has no place in the table to a function in the interp-do-something interface is an error. The interpreter is in the Down state initially.

Table 4. Interpreter State Transitions

\begin{tabular}{|l|l|l|}
\hline \multicolumn{1}{|c|}{ In state } & \multicolumn{1}{|c|}{ System May Call } & \multicolumn{1}{c|}{ Next state } \\
\hline \hline Down & interp_init & Ready \\
\hline Ready & interp_exit & Down \\
\hline Ready & interp_open_program & Working \\
\hline Working & interp_execute_next & Working (if call returns OK) \\
\hline Working & interp_execute_next & Finished (if call returns EXIT) \\
\hline Working & interp_close_program & Ready \\
\hline Finished & interp_close_program & Ready \\
\hline
\end{tabular}

\section{int interp_close_program()}

This closes the current program. The return value is $0(\mathrm{OK})$ or -1 (ERROR). It is an error to call this function if no program is open. It is OK to close a program even if it has not been executed to the end.

\section{int interp_execute_next ()}

This causes the interpreter to decide which statement to execute next and to execute it. Execution of a statement always results in one or more calls to functions in the set of functions that tells the rest of the system what to do. The return value is $0(\mathrm{OK}),-1$ (ERROR), or 1 (EXIT). EXIT is returned only when ENDFIL is interpreted. It is an error to call this function if a program is not open, or an earlier call to this function returned EXIT and the same program is still open.

\section{int interp_exit ()}

This causes the interpreter to exit. This "undoes" interp_init. The return value is $0(\mathrm{OK})$ or -1 (ERROR). It is an error to call this function if a program is open. It is OK to follow a call to interp_exit with a call to interp_init. 
This causes the interpreter to get ready to run. Once this function has been called and has returned $\mathrm{OK}$, the interpreter stays initialized until a call to interp_exit. The return value is $0(\mathrm{OK})$ or -1 (ERROR). It is an error to call this function if the interpreter has already been initialized.

\section{int interp_open_program (char * dmis_file_name)}

This causes the interpreter to open the named DMIS file, so that it is ready to be executed. The file stays open until a call to interp_close_program. The return value is 0 (OK) or -1 (ERROR). If the file cannot be opened (if it does not exist, for example), ERROR is returned. It is an error to call this function if a file is already open or if the interpreter has not been initialized.

\section{B.4 Functions to Tell the Rest of the System What to Do.}

\section{B.4.1 Discussion and Issues}

Functions which tell the rest of the system what to do are intended to be atomic. In other words, each function does one thing only. DMIS statements are not all atomic. Thus, executing one DMIS statement will often result in more than one of these functions being called.

A recurring issue for canonical functions is: For a given functionality, should the interpreter handle it internally or should the interpreter assume the system which executes canonical functions will handle it? A typical example is length units (suppose the alternatives in the input are inches and millimeters). If the executing system always expects millimeters, the interpreter should turn on an internal converter when it reads a DMIS statement that says to use inches. Thereafter, all lengths should be converted to millimeters by the interpreter before being used in canonical commands. If the executing system can handle different units itself, then there are two choices: either the interpreter can do the conversion or the interpreter can make the function call: USE_LENGTH_UNITS(CANON_UNIT_INCH); that function is not even defined in the previous case. One criterion for deciding where to put functionality is that most commercially available CMM controllers should have the functionality if a canonical command can call for it.

A compromise position could be taken where the functionality is shared between the interpreter and the CMM. For example, we might allow only two canonical length units (inches and millimeters), convert feet to inches in the interpreter, and convert centimeters and meters to millimeters in the interpreter. Currently, there is no sharing; the interpreter assumes the CMM can handle all five length units.

A closely related problem is what to do when DMIS allows symbolic values for items which are normally numeric. For example, DMIS allows HIGH, LOW, and DEFALT for feed rates. The interpreter will convert such symbolic values to numeric values by referring to configuration data which is read in at initialization time. If the CMM may or must be given a symbolic value, the controller which gives commands to the CMM can convert a numeric value back to a symbolic value when the canonical command is converted to the CMM's native language. That controller can get the right symbolic value by referring to the same configuration file.

A DMIS program may define many instances of DMIS types such as features, sensors, and tolerances. A major issue is what view should the executing system have of these things.

SENSORS - Since the executing system must manipulate the sensors, we assume here that the 
system can identify sensors by name and can remember any number of sensor names.

FEATURES - We assume the executing system knows nothing about the feature types defined in DMIS.

TOLERANCES - like features

DATUMS - like features

Output - The DMIS spec view [CAM-I, p.332] is that several methods of information output may be attached to a system running a DMIS program (CRT, magnetic storage (disk file), paper printer, or communications port), and the output format may be either DMIS format or a vendor format. It is assumed in these canonical functions that the DMIS interpreter will handle all output in DMIS format (since the interpreter is supposed to be the DMIS expert). Thus, there should never be any canonical functions for producing DMIS output. This version of the canonical functions does not deal with vendor format output, either. Future versions of these canonical commands should deal with vendor format output.

This set includes some functions (currently only CATCH_UP) which incorporate a view of how the rest of the system works.

\section{B.4.2 Types}

typedef int CANON_PIANE
\#define CANON_PLANE_XY 1
\#define CANON_PLANE_YZ 2
\#define CANON_PLANE_XZ 3

[CAM-I, p. 122] allows these planes. CANON_PLANE is used in the SET_PLANE function to identify the plane to use.

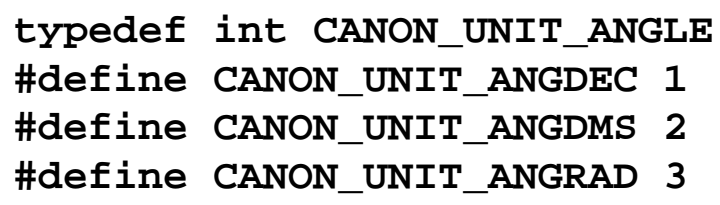

[CAM-I, p. 180] allows these angle units. ANGDEC is angle in degrees with a decimal, e.g. 34.0779. ANGDMS is angle in degrees, minutes, and seconds (all integer), e.g. 4:03:47, but this form is currently not supported by the interpreter. ANGRAD is angle in radians, e.g. 4.1976. CANON_UNIT_ANGLE is used in the USE_ANGLE_UNITS function.

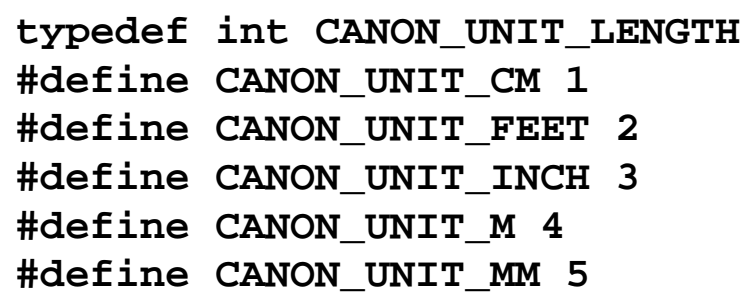

[CAM-I, p. 180] allows these length units. CM is centimeters, FEET is feet, INCH is inches, M is meters, MM is millimeters. CANON_UNIT_LENGTH is used in the USE_LENGTH_UNITS 
function.

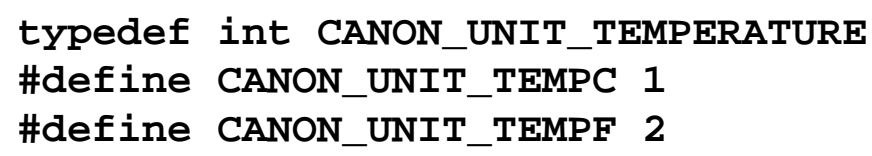

TEMPC is degrees centigrade. TEMPF is degrees Fahrenheit. [CAM-I, p. 180] allows these temperature units. CANON_UNIT_TEMPERATURE is used in the USE_TEMPERATURE_UNITS function.

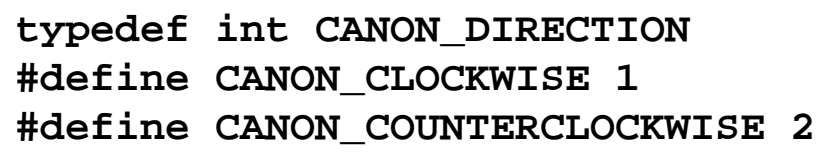

CANON_DIRECTION is for the direction of rotation of a rotary table. It is used in the ROTATE_TABLE function.

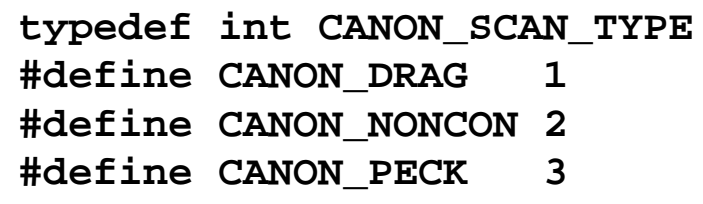

CANON_DRAG means a contact probe is dragged along the surface. CANON_NONCON means the probe is moved near the surface but not touching it (non-contact). CANON_PECK means the probe is primarily moved near the surface but not touching it and from time to time stops moving along the surface and moves to touch the surface and retract. [CAM-I, p. 245] allows these scan types. CANON_SCAN_TYPE is used in the SET_SCAN_TYPE function.

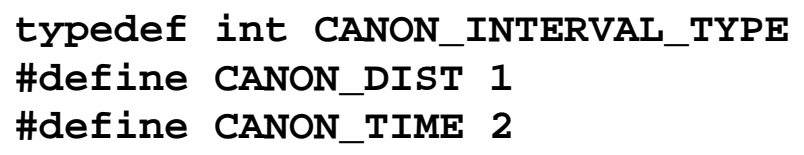

DIST is distance in current units. TIME is time in seconds. [CAM-I, p. 245] allows these scan interval types. CANON_INTERVAL_TYPE is used in the SET_SCAN_INTERVAL_TYPE function.

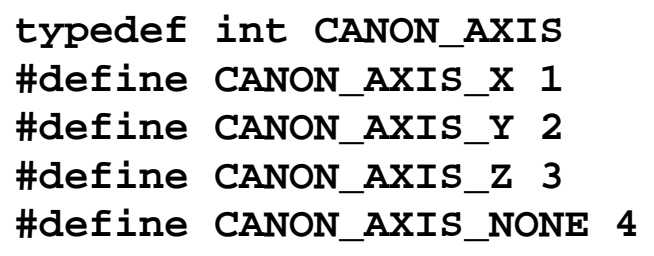

These are the axis types needed for referring to axes. CANON_AXIS is used in the SET_SCAN_DIST_INTERVAL function. 


\section{B.4.3 Functions}

\section{void ADVISORY (char * message)}

This indicates the interpreter has changed something internally in the course of executing a DMIS statement. The CMM controller should do nothing when this function is called. It is provided only to show that a statement has been executed and to allow a human-interpretable description of what the interpreter did.

\section{vOId ASSIGN_SENSOR_TO_SLOT \\ (char * sensor_name, int slot_number)}

This tells the executing system that the named sensor is in the numbered changer slot. The executing system is not required to do anything in response to this command (not even remember the correspondence, although it may do that). This command is for executing THLDEF [CAM-I, p. 166]. See discussion of CHANGE_SENSOR below.

\section{void CATCH_UP ()}

The interpreter expects that the actions specified by all function calls in this section will be carried out as described in the order in which the calls are made (or possibly in parallel if the effects are the same). The interpreter does not usually know when the actions are carried out. In many cases the interpreter needs data collected as a result of the actions, so the interpreter needs to know that the actions have been carried out. The CATCH_UP function is provided to deal with this situation. After the CATCH_UP function is called by the interpreter, the rest of the system should not call the interpreter again until all previous actions specified in canonical function calls have been executed.

Currently, the interpreter is not trying to check that a CATCH_UP has been carried out. It might be useful to have the rest of the system notify the interpreter that it is caught up.

If the operation of the rest of the system is such that actions are carried out immediately after a function call is made (before doing anything else), which is one standard mode of operation, executing CATCH_UP is a null operation; the system is always caught up. If the rest of the system is queuing actions, however, the queue should be emptied before the interpreter is called again.

On the next call by the executing system to interp_execute_next, following a call by the interpreter to CATCH_UP, the interpreter will call one or more functions asking for data before making any calls to the functions in this section.

The functionality of CATCH_UP could be obtained, alternatively, by having the interpreter return a value that means catch up from a call to one of the functions in the interp_do_something interface. This is the way it was implemented in EMC machining center controllers. Making CATCH_UP a function is just a higher-profile method.

\section{void CHANGE_SENSOR (char * sensor_name)}

It is assumed that only one sensor is used by a CMM at a time. This changes the sensor to the one named in function call. If the named sensor is already being used, that is OK and nothing need be 
done.

The sensor_name argument must be the name of a sensor previously defined with SNSDEF. Since the interpreter maintains information about sensors defined with SNSDEF, it is anticipated that the rest of the system may make canonical function calls to the interpreter to get this information.

Changing the sensor should also automatically change system variables which depend upon the definition of the sensor, such as the current location.

Alternatively, tool holder slot number might be used as an argument to CHANGE_SENSOR, and each sensor would be identified with a slot number. If the CMM did not have slots, the sensors could still be numbered. DMIS includes the THLDEF (tool holder definition) statement to support the use of numbered slots. It would be feasible to require that THLDEF be used before a SNSLCT statement referring to that sensor. However, existing DMIS programs (teampart.dmis, in particular) do not always use THLDEF. Some systems are smart enough to keep track of sensors (or other objects) without having to put them in the same place all the time. This can save a lot time in tool changes. Forcing such systems to use slot numbers would work against this desirable feature.

Another alternative would be to have both slot number and name as arguments to CHANGE_SENSOR. If the slot number were non-negative, it would be intended to be a real slot number. It it were negative, that would indicate that it is not a valid slot number and should be ignored. The name would be either an empty string or the correct name when a non-negative slot number was used, and would be the correct name if a negative slot number was used. One or both of the slot number and name would have to be correct.

An additional consideration is that the assignment of sensors to slots may change even when the inspection program does not change. If sensors are identified by name only in a program, changing slots is not a problem. The executing system may have its own data about the location of sensors, which is not included in the program. This is common practice in the closely related field of machining. If the interpreter needs this information, it can be downloaded to the interpreter at initialization time (what the NIST RS274 interpreters do [Kramer]), or the interpreter might ask for it when it is needed (not implemented).

\section{void DEFINE_SENSOR (char * sensor_name, double x_offset, double y_offset, double z_offset, double tip_diameter)}

The $\mathrm{x}, \mathrm{y}$, and z_offsets of this function locate the center of the tip of the named probe with respect to the coordinate system of the probe. It also provides the diameter of the probe tip, which is assumed to be spherical.

The coordinate system of the probe is expected to be known.

It is suggested, but not required, that the coordinate system of the probe be such that, when the probe is attached to a CMM in a normal manner, the origin of the probe coordinate system should be at the mount location of the CMM, and the axes of the probe coordinate system should be parallel to the corresponding axes of the CMM. This location will simplify the transformations needed for deriving CMM axis positions from desired probe positions. 
vOid LOGGING_OFF ()

This turns logging point data OFF.

void LOGGING_ON (char * log_name)

This turns logging point data ON. If SCAN_TO_POSE is called when logging point data is ON, the data points taken during the scan are saved under the name log_name (normally a file name but not required to be a file). If there are already some points saved under that name, they are preserved, and the new points are added after the old ones. It is an error to call LOGGING_ON twice without an intervening LOGGING_OFF.

VOId MEASURE_POINT

(double $x$, double $y$, double $z$, double $i$, double $j$, double $k$ )

This is called for executing the DMIS PTMEAS statement [CAM-I, p. 208] when SCNMOD is OFF.

All the parameters to this command and the points and vectors defined below refer to the currently active coordinate system.

The following should happen when this function is called. These actions are supposed to be what is intended by the DMIS spec. Because the spec is vague, however, it is hard to be sure this is what the spec intends. It may be that the spec is vague intentionally, to allow for various implementations.

\section{SETUP}

Let $\mathrm{C}$ be the location of the controlled point (center of probe tip) before this instruction is executed.

Let $\mathrm{P}=(\mathrm{x}, \mathrm{y}, \mathrm{z})$ be the nominal location of the point to be probed. $\mathrm{P}$ must lie on a surface, but the system is not required to check this.

Let $\mathrm{V}=(\mathrm{i}, \mathrm{j}, \mathrm{k})$ represent a vector. If the surface has a normal at $\mathrm{P}, \mathrm{V}$ should point in that direction, but no verification of this is required. If the surface normal is not defined (the tip of a cone, for example) $\mathrm{V}$ should point away from surface at $\mathrm{P}$ (preferably into a region where a normal to the surface in the vicinity of $\mathrm{P}$ cannot be drawn, but this is not required). If $\mathrm{V}$ is not a unit vector (within the system's tolerance for unit vectors), probe status is set to CANON_BAD, and execution is finished (doesn't even start, actually).

Let $\mathrm{A}$ be a point lying in the direction of $\mathrm{V}$, one approach distance (as given with SNSET [CAMI, p. 150] or by default) from $P$.

Let $\mathrm{T}$ be the location of the controlled point when the probe trips, if it trips during execution of this command.

Let $\mathrm{S}$ be a point lying in the direction opposite $\mathrm{V}$ one search distance (as given with SNSET or by default) from $P$.

\section{ACTION}

1. Move the controlled point in a straight line from $\mathrm{C}$ to $\mathrm{A}$ at the given feed rate (whatever the 
system can do to control acceleration and deceleration is assumed to be adequate). If the probe trips during this move, probe status is set to CANON_BAD, and execution is finished. It may be desirable to allow traverse rate here because it is common for lots of points to be probed one after the other, and using traverse rate could save a lot of time. On the other hand, using the feed rate may help prevent broken probes, since it may be possible to stop quickly enough to avoid breaking a tripped probe if it is moving at feed rate where it would not be possible if it were moving at traverse rate.

2. Move the controlled point in a straight line from $A$ towards $S$ at feed rate.

Stopping at the intersection of lines CA and AS (point A) is optional.

If the probe trips during this move before it is within the system's tolerance for feed rate or direction, probe status is set to CANON_BAD, and execution is finished.

If the probe does not trip by the time it reaches $\mathrm{S}$, it is stopped at maximum normal deceleration and moved back to S, probe status is set to CANON_BAD, and execution is finished.

3. Otherwise, if the probe trips during the move from A towards $\mathrm{S}$, it is stopped at maximum normal deceleration and moved at feed rate to $\mathrm{T}$ (might change to traverse rate).

4. Without stopping, the probe is moved at feed rate (might change to traverse rate) in the direction of $\mathrm{V}$ one retract distance (as given with SNSET or by default) from $\mathrm{T}$. If the probe trips during this move, probe status is set to CANON_BAD, and execution is finished. Otherwise, probe status is set to $\mathrm{OK}$.

After this command has been executed $\mathrm{OK}$, the executing system must have recorded the following - which must be available for reading out at least until the next command that moves the probe is executed.

1. the coordinates of the final position of the probe.

2. The coordinates of a point. The manual [CAM-I, p. 177] implies strongly that:

a. If probe compensation is on (as determined by the PRCOMP DMIS statement), this should be the system's best estimate of the actual coordinates of $\mathrm{P}$.

b. If probe compensation is off, this should be the coordinates of $\mathrm{T}$.

The approach, retract, and search distances could be parameters to the MEASURE_POINT command, rather than being assumed available within the system.

If the probe is not a touch trigger probe, a lot of the above does not make sense. The meaning of this command for other than a touch trigger probe should be rethought.

void MESSAGE (char * text)

This indicates that a message is to be displayed for the operator. The nature of the display is up to the receiving system, but will normally be on a computer monitor that is visible to the operator.

VOId PROBE_RADIUS_COMPENSATION_OFF ()

vOid PROBE_RADIUS_COMPENSATION_ON ( ) 
The model of a probe tip (assumed both in the canonical commands and in DMIS [CAM-I, p. 126]) is that the tip of a touch trigger probe is a sphere, cylinder, or disk with its center at a known distance and direction from a point at the base of the probe. The diameter of the sphere, cylinder, or disk is given with the SNSDEF statement. In the case of a cylinder or disk, the orientation of the axis is also known.

These commands turn probe radius compensation off and on for a touch trigger probe. We assume that the controlled point is the center of the probe tip.

When probe radius compensation is off and a probing is made, the location of the center of the probe tip at the time the probe is tripped should be reported. The manual [CAM-I, p. 177] refers to this as "raw data." Raw data is not defined in the glossary of the manual.

When probe radius compensation is on, the system's best estimate of the location of the contact point that caused the trip should be reported.

\section{VOId PROGRAM_END ()}

A call to PROGRAM_END signals the end of a program. After this function has been called, the only function from the cmm_do_something interface which is valid to call is PROGRAM_START. The executing system is not required to do anything to carry out the PROGRAM_END function, but may perform termination actions if needed.

\section{void PROGRAM_START (char * text)}

PROGRAM_START carries out the DMISMN statement [CAM-I, p. 170], which must be the first statement of a DMIS program.

A call to this function signals the beginning of a program. It is an error to call this function two times without an intervening call to PROGRAM_END. The executing system does not have to do anything to carry out this function, but it is likely that some sort of initialization will be carried out in most executing systems.

The text supplied with DMISMN is arbitrary. It is passed on by the PROGRAM_START function in case the executing system wants to do something with it.

vOId ROTATE_TABLE

(double position, CANON_DIRECTION wiseness)

ROTATE_TABLE assumes that there is one rotary table, and it may be turned to a given position, which is assumed to be in current angle units (degrees or radians) from a home position. Position values range $0<=$ angle $<360$ for degrees and $0<=$ angle $<2 \mathrm{Pi}$ for radians. Values of position outside this range are illegal. The wiseness must be CANON_CLOCKWISE or CANON_COUNTERCLOCKWISE.

Wiseness is as viewed from the side of the table on which the workpiece is usually fixtured.

The motion should be a smooth acceleration from the start position to the programmed rotational velocity and a smooth deceleration to a stop at the end position.

The DMIS language allows for any number of rotary tables, but these cmm_canonical functions 
allow for only one.

A command to rotate to the current nominal position should result in one full revolution of the table in the stated direction. The executing system must keep track of current nominal position, as given by ROTATE_TABLE commands.

\section{vOId SCAN_TO_POSE}

\section{(double $x$, double $y$, double $z$, double $i$, double $j$, double $k$ )}

This is called for executing the DMIS PTMEAS statement [CAM-I, p. 208], when SCNMOD is ON.

This command is intended to be implementable for touch trigger probes doing peck probing, for position probes which can be dragged along a surface, or for non-contact probes which might be moved along near a surface (either servoed to stay near the surface or not).

All the parameters to this command and the points and vectors defined below refer to the currently active coordinate system.

The following should happen when this function is called. These actions are supposed to be what is intended by the DMIS spec, but the spec is vague, so it is hard to be sure this is what the spec intends.

\section{SETUP}

Let $\mathrm{C}=(\mathrm{Cx}, \mathrm{Cy}, \mathrm{Cz})$ be the location of the controlled point (center of probe tip) before this instruction is executed.

Let $\mathrm{P}=(\mathrm{Px}, \mathrm{Py}, \mathrm{Pz})$ be the nominal location of the goal point (given by $\mathrm{x}, \mathrm{y}$, and $\mathrm{z}$ in the function prototype above).

Vector $(\mathrm{i}, \mathrm{j}, \mathrm{k})$ represents a unit vector pointing in the intended direction of the probe at the end of the move. If that is not a unit vector (within the system's tolerance for unit vectors), probe status is set to CANON_BAD, and execution is finished (doesn't even start, actually).

\section{ACTION}

Move the controlled point from $\mathrm{C}$ towards $\mathrm{P}$ at the current scan feed rate, taking measurements periodically (either at fixed time intervals or fixed intervals of distance along the line, according to how that has been set).

The motion from C to P should be "straight" in some sense, but is not necessarily a straight line. Exactly what this means is not defined, but the following paragraph gives an example of one type of motion that is intended to be permitted.

Example: A non-contact probe is moved so that the probe tip stays in a plane which contains the line from $\mathrm{C}$ to $\mathrm{P}$ and is parallel to the $\mathrm{Z}$-axis. During this move, the $\mathrm{Z}$ location of the probe tip is controlled so that the it stays near the surface of the part being measured but does not hit the part.

If some problem is detected during this move, (e.g., motion control fails or the probe breaks off) probe status is set to CANON_BAD, and execution is finished. Otherwise, probe status is set to CANON_OK. 
The motion stops when the probe has "reached the goal pose." In other words, position and orientation (or selected components of them) are within some tolerance zone of the goal pose (or selected components of it).

\section{AFTEREFFECTS}

After this command has been executed $\mathrm{OK}$, the executing system must have recorded the coordinates of the final position of the probe, which must be available for reading out at least until the next command that moves the probe is executed.

If data logging is $\mathrm{ON}$, after this command has been executed $\mathrm{OK}$, the executing system must have recorded the coordinates of a number of points in a file of the given name (or using any other method of storing the data so it can be retrieved by name). The file must be available for reading out at any time until a PROGRAM_END command is received. The executing system must also remember how many points there are in the file. The points in the file should be points on the feature being measured. If data logging is OFF, no point data (other than the final position) need be saved.

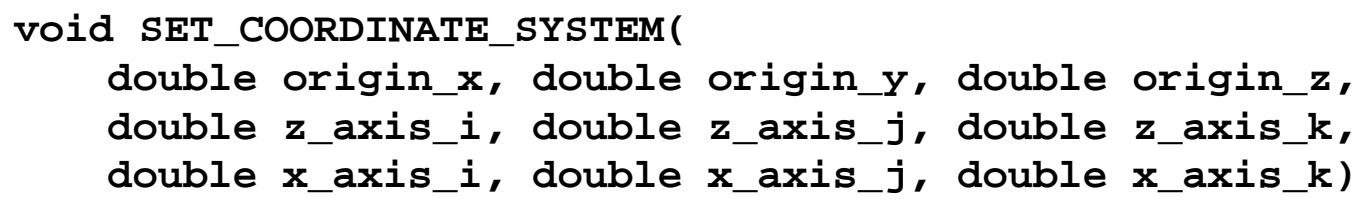

The arguments to this function are in terms of a machine's default coordinate system (the location of which relative to the machine hardware is known).

The arguments describe a coordinate system with its origin at (origin_x, origin_y, origin_z), its Z axis pointing in the direction of the vector (z_axis_i, $\left.z \_a x i s \_j, z \_a x i s \_k\right)$, and its $X$ axis pointing in the direction of the vector (x_axis_i, $\left.x \_a x i s \_j, x \_a x i s \_k\right)$. Those two vectors must be unit vectors within a tolerance of not more than 0.00001; an implementation may require a smaller tolerance.

It will be useful to have more compact notation, as follows (where the last two items are vectors):

$$
\begin{aligned}
& \text { x_axis_i }=X x \\
& \text { x_axis_j }=X y \\
& \text { x_axis_k =Xz } \\
& \text { z_axis_i }=\mathrm{Zx} \\
& \text { z_axis_j }=\mathrm{Zy} \\
& \text { z_axis_k = Zz } \\
& \text { origin_x }=\mathrm{Tx} \\
& \text { origin_y }=\mathrm{Ty} \\
& \text { origin_z }=\mathrm{Tz} \\
& (\mathrm{Xx}, \mathrm{Xy}, \mathrm{Xz})=\mathrm{Vx} \\
& (\mathrm{Zx}, \mathrm{Zy}, \mathrm{Zz})=\mathrm{Vz}
\end{aligned}
$$

Define $\mathrm{Vy}$ as $(\mathrm{Vz} x \mathrm{Vx})$, meaning $\mathrm{Vy}$ is the cross product of $\mathrm{Vz}$ and $\mathrm{Vx}$.

Define the components of $\mathrm{Vy}$ by $\mathrm{Vy}=(\mathrm{Yx}, \mathrm{Yy}, \mathrm{Yz})$. Then, by the definition of cross product: 


$$
\begin{aligned}
& Y x=(Z y \times X z)-(Z z \times X y) \\
& Y y=(Z z \times X x)-(Z x \times X z) \\
& Y z=(Z x \times X y)-(Z y \times X x)
\end{aligned}
$$

The reason this works is that the Y-axis of a coordinate system is the cross product of the Z-axis with the $\mathrm{X}$-axis. If the system is moved, that relationship continues to hold. Vy is the direction in which the transformed Y-axis points.

Now it is easy to build a $4 \times 4$ homogeneous coordinate transform matrix. The matrix is:

$$
\left[\begin{array}{cccc}
X x & Y x & Z x & T x \\
X y & Y y & Z y & T y \\
X z & Y z & Z z & T z \\
0 & 0 & 0 & 1
\end{array}\right]
$$

To convert a point $(\mathrm{x}, \mathrm{y}, \mathrm{z}, 1)$ to its location in the transformed coordinate system, multiply as follows:

$$
\left[\begin{array}{cccc}
X x & Y x & Z x & T x \\
X y & Y y & Z y & T y \\
X z & Y z & Z z & T z \\
0 & 0 & 0 & 1
\end{array}\right]\left[\begin{array}{c}
x \\
y \\
z \\
1
\end{array}\right]
$$

After a call to SET_COORDINATE_SYSTEM, all arguments to cmm_do_something functions (other than SET_COORDINATE_SYSTEM) which are interpreted in terms of a coordinate system, refer to the coordinate system described in the function call, until the end of a program or another call to SET_COORDINATE_SYSTEM.

The selected plane becomes the plane in the new coordinate system with the same name as the selected plane in the previously active coordinate system.

\section{void SET_DISTANCE_APPROACH (double distance)}

This sets the approach distance used in the MEASURE_POINT function. This function and the following four SET_DISTANCE_XXX functions implement the DMIS SNSET command [CAM-I, p. 150].

\section{void SET_DISTANCE_CLRSRF (double distance)}

This sets the clearance from surface distance used in probing. This distance is not currently used by the probing functions.

\section{void SET_DISTANCE_DEPTH(double distance)}

This sets the depth distance used in probing. This distance is not currently used by the probing functions. 
void SET_DISTANCE_RETRACT (double distance)

This sets the retract distance used in the MEASURE_POINT function.

void SET_DISTANCE_SEARCH(double distance)

This sets the search distance used in the MEASURE_POINT function.

void SET_FEED_RATE(double rate)

All four canonical functions for setting feed rates (the others are SET_ROTARY_RATE, SET_SCAN_RATE,SET_TRAVERSE_RATE) take a numerical argument and assume a specific unit. All four are used in implementing the DMIS FEDRAT command [CAM-I, p. 173].

The rate is expressed in millimeters per minute. One alternative would be to have a "SET_VELOCITY_UNITS" command (the DMIS FEDRAT statement provides for setting units).

The feed rate is the constant rate of motion along whatever path is being followed relative to the object being probed. The CMM is expected to do its best to maintain this rate during measuring operations. The rate applies to the controlled point, which is usually the probe tip (maybe it should be the center of a sphere which is assumed to be at the end of the probe).

void SET_PLANE(CANON_PLANE plane)

Use the plane designated by "plane" as the selected plane. The selected plane must be the XYplane, the XZ-plane, or the YZ-plane. This relates to the DMIS WKPLAN statement [CAM-I, p. 122]. The plane refers to one of the three principal planes of the current coordinate system.

void SET_ROTARY_RATE (double rate)

The rate is expressed in RPM of a rotary table relative to the housing or mounting of the rotary table. Also see notes for SET_FEED_RATE.

void SET_ROTARY_ZERO (double angle)

The angle of the rotary table is measured from some reference direction, (which has a default position) at which the angle is zero. This command moves the reference direction to the given angle, which is in terms of the default reference direction and is measured in current angle units (degrees in decimal form or radians). This implements the DMIS ROTSET command [CAM-I, p. 187].

After a call to this function, all arguments to cmm_do_something functions (other than SET_ROTARY_ZERO) which are rotary table angles are measured from the new reference direction, until the end of a program or another call to SET_ROTARY_ZERO.

Any value of "angle" is OK, provided it is a double. 
VOId SET_SCAN_DIST_INTERVAL

(double dist_interval, CANON_AXIS axis)

This functions and the other SET_SCAN_XXX functions, which follow, implement the DMIS SCNSET command [CAM-I, p. 245].

The dist_interval is expressed in current distance units.

The interval and axis are set as given and stay set until the end of the program or until another call to SET_SCAN_DIST_INTERVAL.

If axis is CANON_AXIS_NONE, the distance interval is measured "along the surface of the part from one point to the next." Otherwise the interval is measured "along the specified coordinate axis."

void SET_SCAN_INTERVAL_TYPE(CANON_INTERVAL_TYPE interval_tYPe)

The interval_type is set as given and stays set until the end of the program or until another call to SET_SCAN_INTERVAL_TYPE. The interval_type and its corresponding interval are used for all scanning moves. Data is taken during a scanning move at the end of each interval. The degree of exactness of the interval is implementation-dependent.

void SET_SCAN_RATE (double rate)

The rate is expressed in millimeters per minute.

Also see notes for SET_FEED_RATE.

void SET_SCAN_TIME_INTERVAL (double time_interval)

The time_interval is expressed in seconds.

The interval is set as given and stays set until the end of the program or until another call to SET_SCAN_TIME_INTERVAL.

void SET_SCAN_TYPE(CANON_SCAN_TYPE the_tYPe)

The scan type is set to the given type, which may be CANON_DRAG, CANON_NONCON, or CANON_PECK.

When the type is set to CANON_DRAG, scan moves are executed by dragging the sensor along the surface, in contact with the surface.

When the type is set to CANON_NONCON, scan moves are executed by any non-contact method. Examples of such methods are:

1. moving a capacitance probe along near the surface, but not in contact with the surface.

2. guiding a beam of electromagnetic radiation (light, radio waves, etc.) along the surface, and measuring by detecting reflected radiation.

When the type is set to CANON_PECK, scan moves are executed by moving the sensor along a line from the initial pose to the goal pose and intermittently moving the probe to touch the surface 
(with a touch trigger probe, for example) or to approach the surface but not touch it (with a capacitance probe, for example).

void SET_TRAVERSE_RATE (double rate)

The rate is expressed in millimeters per minute.

Also see notes for SET_FEED_RATE.

The traverse rate is the constant rate at which the CMM tries to move the controlled point during goto moves.

void STRAIGHT_TRAVERSE (double $x$, double $y$, double $z$ )

Move at traverse rate in a straight line from the current point to the final XYZ position given by $\mathrm{x}$, $\mathrm{y}$, and z. Do not change the orientation of the probe during the move. This implements the DMIS GOTO command [CAM-I, p. 203]

vOId USE_ANGLE_UNITS (CANON_UNIT_ANGLE u)

Use the specified units for angle. The units must be one of the CANON_UNIT_ANGLE (see above). This function and the other two USE_XXX_UNITS functions, which follow, implement the DMIS UNITS command [CAM-I, p. 180].

vOId USE_LENGTH_UNITS (CANON_UNIT_LENGTH u)

Use the specified units for length. The units must be one of the CANON_UNIT_LENGTH (see above).

VOId USE_TEMPERATURE_UNITS (CANON_UNIT_TEMPERATURE u)

Use the specified units for temperature. The units must be one of the CANON_UNIT_TEMPERATURE (see above). The interpreter currently makes no use of temperature, so there is not much point in setting units.

\section{B.5 Functions to Get Feature Parameters from Arrays of Points.}

These functions are for the interpreter to call to get feature parameters from arrays of points. The points for a feature are assumed to be expressed in the same coordinate system as the one in which the nominal feature was defined. This is not necessarily the same as the currently active coordinate system.

There is one function for each implemented feature type defined in DMIS (circle, cylinder, line, plane, point).

Each function has an integer return value, which is to be used to pass status back to the calling function (either OK or ERROR). OK should be returned if the function was able to do its work without error. ERROR should be returned if the function detects problems, such as not enough 
points being provided to fully determine the feature.

Each function has two sets of parameters: input parameters and input/output parameters. All of these appear as arguments to the function.

Each function has three input parameters:

a. a pointer to an array of triples of $(\mathrm{C}++)$ doubles. Each triple represents a point $(\mathrm{x}, \mathrm{y}, \mathrm{z})$.

b. an integer giving the number of points in the array to use.

c. a double giving the input_tolerance.

Each function has several input/output parameters, all of which are pointers to doubles and represent the parameters of a feature of the given type. Each of these must be set to its nominal value before the function is called. Each feature extraction function has the choice of using the nominal data or not using it. Likewise, each function may use the input_tolerance or not use it. Where several parameters are the components of a "normal" vector or a "direction" vector, these vectors must be unit vectors (within an implementation-dependent tolerance).

If the function returns $\mathrm{OK}$, the values of these output doubles should have been set by the function. If the function returns error, the values of these doubles are meaningless after the function returns.

These functions are not required to make an effort to satisfy any constraints. It might be useful to add parameters to identify constraints. The most obvious constraint is to require that the sum of the squares of the errors be minimized. Another reasonable constraint would be to require the function to try to minimize the maximum error. A third possibility is to use the nominal values to constrain the assignment of output parameters. For example, if all points lie within the input_tolerance of the nominal feature, the function might be constrained to return the nominal feature.

It might be useful to allow several alternative functions where only one is prototyped here. This could be done by adding a parameter which is the name (or other identifier) of a function which can do the required work.

It would also be feasible to have a single function to do the work of all the functions here. It would have additional input arguments to indicate the feature type and other things. The meaning of the output arguments would vary according to the input feature type. This does not seem like a good thing to do.

There are other interesting issues.

1. The DMIS ALGDEF statement allows the naming of algorithms in an array of algorithms, and the GEOALG statement allows assigning an algorithm (identified by type names) for use in generating parameters for features of a given type from point sets. These have not been implemented but might be.

2. DMIS allows for the simultaneous determination of all parameters of an "actual" feature, but does not allow for the parameters to be given actual values one at a time. This is very strange. The DMIS OBTAIN statement allows a variable to be given the value of a parameter of a feature. What is needed is the reverse operation (NIATBO?) of giving a single parameter of a feature an actual value. If NIATBO were available, measurement algorithms could be readily written in 
DMIS. As DMIS is, it is hard to write measurement algorithms in DMIS.

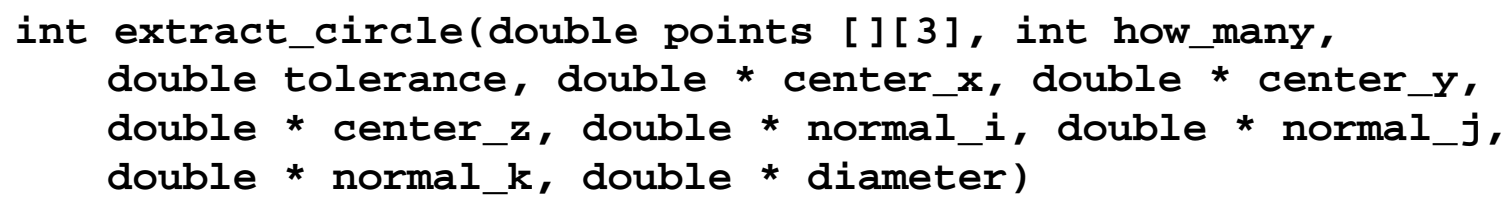

extract_circle finds a circle fit to the points. It produces the coordinates of the center of the circle, the direction of the normal to the plane of the circle, and the diameter of the circle.

int extract_cylinder(double points [] [3], int how_many, double tolerance, double * point_x, double * point_y, double * point_z, double * direction_x, double * direction_y, double * direction_z, double * diameter)

extract_cylinder finds a cylinder fit to the points. It produces the coordinates of a point on the axis of the cylinder, the direction of the axis, and the diameter of the cylinder.

int extract_line(double points [] [3], int how_many, double tolerance, double * point_x, double * point_y, double * point_z, double * direction_x, double * direction_y, double * direction_z)

extract_line finds a line fit to the points. It produces the coordinates of a point on the line and the direction of the line.

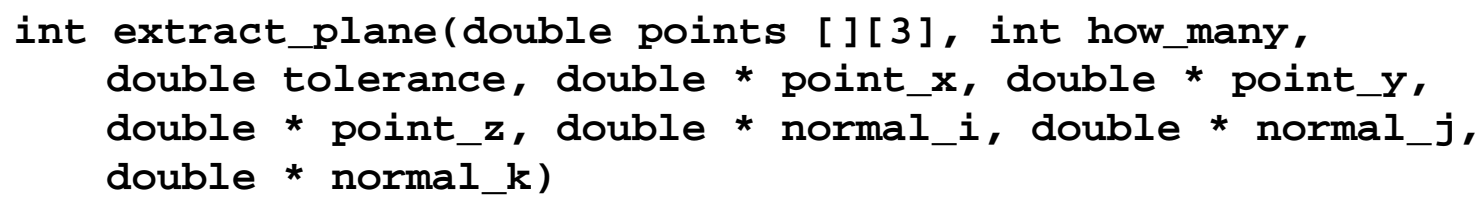

extract_plane finds a plane fit to the points. It produces the coordinates of a point on the plane and a normal to the plane.

int extract_point (double points [] [3], int how_many, double tolerance, double * point_x, double * point_y, double * point_z)

extract_point finds a single point fit to the data points. 


\section{Appendix C Building a Stand-Alone Executable}

On a SUN SPARCstation 20, an executable file for the stand-alone interpreter may be built from source code in under four minutes, as described below. The same procedure should work on any computer running a Unix operating system that has the standard C++ libraries and the STEPTools library. On computers running other operating systems, compilation should also be easy, provided the standard $\mathrm{C}++$ libraries and STEPTools library are available.

To make an executable, 12 source code files must be placed in the same directory along with the Makefile shown in Table 5 below. The source code files are:

cmm_canon.cc - function definitions for four of five interfaces to interpreter

cmm_canon.hh - header file for all interfaces to interpreter

cmm_canon_extract1.cc - function definitions for extract_feature interface to interpreter

dmis_schema.cc - access functions for in-core DMIS program structure

dmis_schema.h - header file for in-core DMIS program structure

dmis_schema.rose - STEPTools format file for in-core DMIS program structure

driver.cc - user interface

fit.hh - header file for feature extraction functions

interp.cc - function definitions for interpreter kernel

interp.hh - header file for interpreter kernel

rest_world.hh - header file for dummy world model

tk_common.hh - header file with constants and aliases for clearer programming

An executable file named "dmis" is built in the same directory by giving the command:

\section{make dmis}

To use the Makefile, the definitions of COMPILE, STEP_INCLUDE, and STEP_LIBS must be changed to be correct in the environment where the compilation is being done.

In the Makefile, we are using the Centerline $\mathrm{C}++$ compiler. The $\mathrm{Gnu} \mathrm{C}++$ compiler has also been used. Different versions of the STEPTools library (compiled with the Centerline or Gnu compiler) must be used with different compilers; both versions are supplied by STEPTools, Inc. 
STEP_INCLUDE $=-$ I/depot/step_toolkit/steptools_1.4/include

COMPILE $=\mathrm{CC}-\mathrm{c}-\mathrm{v}-\mathrm{g}-\mathrm{O}$

LINK $=\mathrm{CC}-\mathrm{V}$

DMIS_O $=$ driver.o interp.o cmm_canon.o cmm_canon_extract.o

STEP_LIBS =/depot/step_toolkit/steptools_1.4/lib/solaris/librose_cfront.a

cmm_canon.o: cmm_canon.cc cmm_canon.hh interp.hh

\$(COMPILE) \$(STEP_INCLUDE) cmm_canon.cc

cmm_canon_extract.o: cmm_canon_extract1.cc fit.hh interp.hh cmm_canon.hh

\$(COMPILE) +a1 -o cmm_canon_extract.o \$(STEP_INCLUDE) \

cmm_canon_extract1.cc

dmis: \$(DMIS_O) dmis_schema.o

\$(LINK) -o dmis \$(DMIS_O) dmis_schema.o \$(STEP_LIBS) -lm

dmis_schema.o: dmis_schema.cc dmis_schema.h

\$(COMPILE) \$(STEP_INCLUDE) dmis_schema.cc

driver.o: driver.cc rest_world.hh cmm_canon.hh tk_common.hh

\$(COMPILE) \$(STEP_INCLUDE) driver.cc

interp.o: interp.cc dmis_schema.h interp.hh cmm_canon.hh tk_common.hh

\$(COMPILE) \$(STEP_INCLUDE) interp.cc

Table 5. Makefile for Interpreter 


\section{Appendix D Transcript of a Session}

This is a transcript of a session using the stand-alone interpreter. Characters entered by the user are shown in boldface. All user input is followed by a carriage return not shown here. Several messages always generated by STEPTools are not shown here.

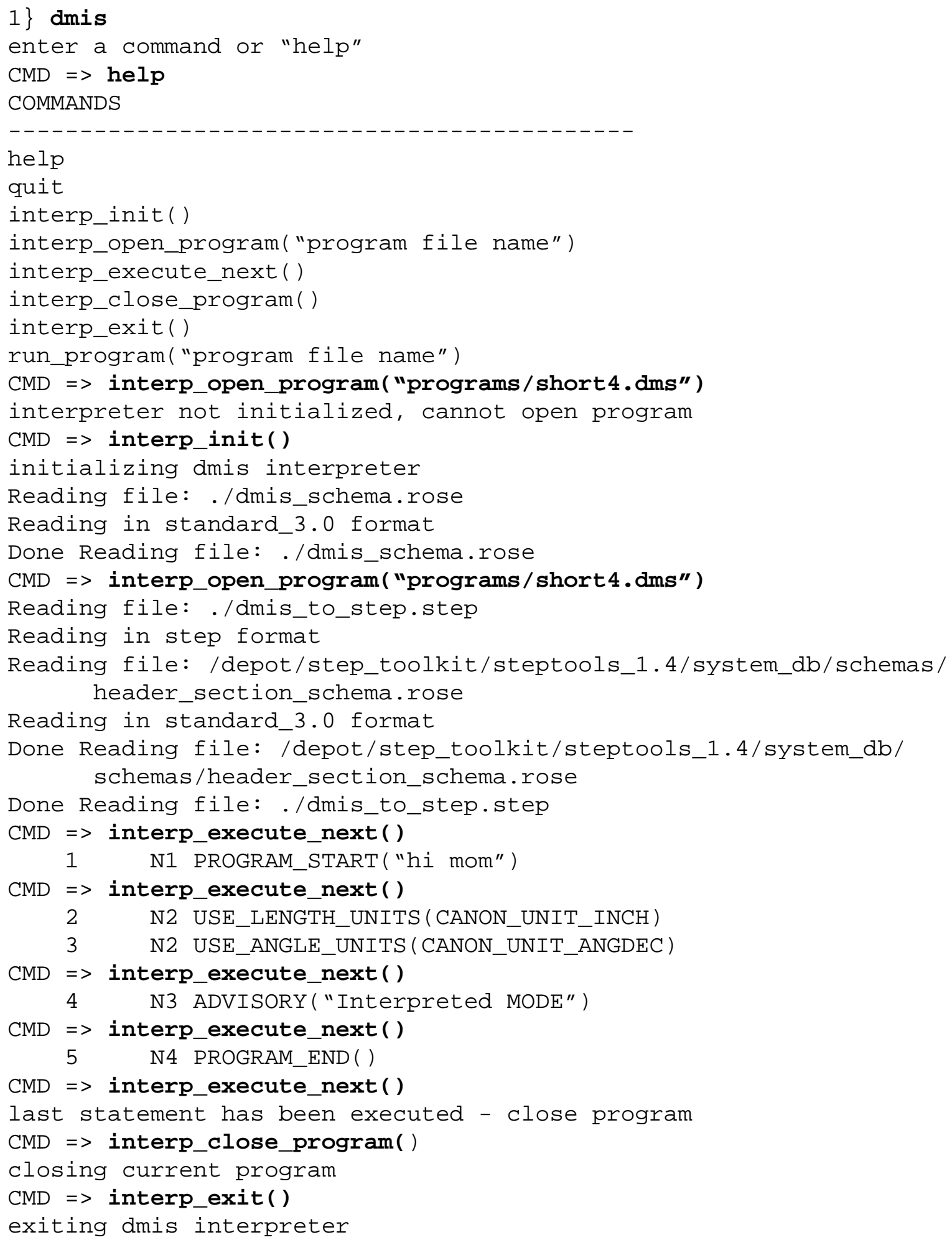




\section{Appendix E Error Handling and Error Messages}

\section{E.1 Error Handling}

The interpreter detects and flags most kinds of illegal input. For example, unreadable input, missing words, extra words, out-of-bounds numbers, and illegal combinations of words are all detected. The interpreter does not check for axis overtravel or excessively high feeds or speeds, or situations where a legal command does something unfortunate, such as crashing a probe.

The basic approach to error handling is:

1. Check carefully for errors.

2. If an error occurs, identify it specifically so that the user can be informed.

3. If an error occurs, return through the function call hierarchy rather than jumping out of it.

Except in the parser, interpreter kernel functions do not report errors directly (that is, they do not call an error reporting routine). Rather, if a kernel function detects an error itself, the function stops where it is, records the error, and returns error to the function that called it. If a subordinate kernel function called by a superior kernel function returns an error code, the superior stops where it is and returns error to the function that called it. When error is returned to an interp_do_something function, the recorded error is reported and error is returned to the caller.

\section{E.2 Types of Error Messages}

\section{E.2.1 Parser Error Messages}

Most of the error messages are sent by interpreter kernel functions when bad input or bad sensory data is detected. These messages are in two categories: those generated by the parser and those generated by the rest of the kernel.

Most DMIS program syntax errors will cause an error in the parser. When this occurs, the parser will print two lines. The first line describes what the parser was looking for when the error occurred. The second line is the DMIS line on which the error occurred. For example, if "ANGDEC" is misspelled as "ANGDEW" in a UNITS statement, the following two lines are printed.

parse error, expecting 'ANGDEC' or 'ANGDMS' or 'ANGRAD' UNITS/MM, ANGDEW

In addition to error messages of the above sort, the parser may give the following error messages. If one of these errors is encountered, the line on which the error occurred will also be printed.

array is full, cannot continue

direction or DAT used twice in DATSET

feature array is full, cannot continue

feature feature id doubly defined

item item id doubly defined

reference to $i d$ not found

same origin used twice

stack overflowed

two FILNAM lines used 
two UNITS lines used

wrong number of point measurements

XDIR and X_DIR used in DATSET

XORIG used twice in DATSET

YDIR and Y_DIR used in DATSET

YORIG used twice in DATSET

ZDIR and Z_DIR used in DATSET

ZORIG used twice in DATSET

A few types of input error will result in lexical scanner error messages, which are short and do not explain much.

\section{E.2.2 Kernel Error Messages}

Error messages generated by the kernel which are not parser messages are listed in Appendix E.3. Most of the messages represent additional checks on the input which the parser will not detect. Some of the messages should never be triggered by any input because the errors for which they check will be detected sooner by the parser. Most of the checks which result in never triggered error messages are made by the "else" at the end of an "if, else if, ..., else if, else" construct in the C++ source code, where the "if" and "else if" $s$ " are intended to be exhaustive of all possibilities.

\section{E.2.3 STEPTools Error Messages}

The STEPTools Part 21 file reader always prints several lines of text when it runs, which happens once per program. Often the file reader also emits warning messages. These warning messages usually say that certain list types were not predefined and had to be defined on the fly. Such messages may be ignored. Other warnings from STEPTools should not appear during file reading.

\section{E.2.4 Driver Error Messages}

The interpreter driver, which is used in the stand-alone interpreter only, also has a few input error messages. They appear if the user gives an inappropriate command and are not covered here.

\section{E.2.5 Feature Extraction Error Messages}

The extraction routine for each feature type has one error message, which simply says the routine failed.

\section{E.2.6 Interp_do_something Error Messages}

The interp_do_something functions include one error message, "probing failed," which will be printed if probing fails.

\section{E.3 Interpreter Kernel Error Messages}

This is a list of all 109 error messages in the interpreter kernel. The list is arranged alphabetically. Messages are in boldface type. Following each message is the name of the function or functions in which it is found, printed in italics. A percent sign indicates that some variable value will appear in that place when the message is printed.

1. $\%$ contains more than one program $\ldots \ldots \ldots \ldots \ldots \ldots \ldots \ldots \ldots \ldots \ldots \ldots$. $\ldots \ldots \ldots$ step 


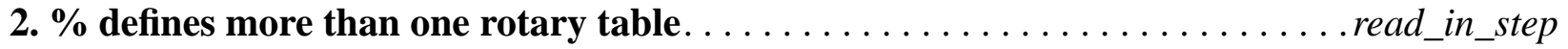

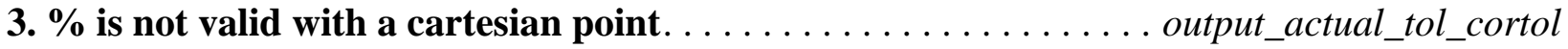

4. $\% \mathrm{~s}$ is not valid with a polar point ................ output_actual_tol_cortol

5. actual circle does not exist $\ldots \ldots \ldots \ldots \ldots \ldots \ldots \ldots \ldots \ldots \ldots \ldots \ldots \ldots \ldots \ldots \ldots$ outpactual_circle

6. actual cylndr does not exist $\ldots \ldots \ldots \ldots \ldots \ldots \ldots \ldots \ldots \ldots \ldots \ldots \ldots \ldots$ outpactual_cylndr

7. actual line does not exist ............. find_rotation_angle, output_actual_line

8. actual plane does not exist $\ldots \ldots \ldots \ldots \ldots \ldots \ldots \ldots \ldots \ldots \ldots \ldots \ldots \ldots$ outpactual_plane

9. actual point does not exist $\ldots \ldots \ldots \ldots \ldots \ldots \ldots \ldots \ldots \ldots \ldots \ldots \ldots \ldots \ldots \ldots \ldots$ outpactual_point

10. actual x-origin plane does not exist $\ldots \ldots \ldots \ldots \ldots \ldots \ldots \ldots \ldots \ldots \ldots \ldots \ldots$ buldransform_dat

11. actual y-origin plane does not exist $\ldots \ldots \ldots \ldots \ldots \ldots \ldots \ldots \ldots$ buld_transform_dat

12. actual z-origin plane does not exist $\ldots \ldots \ldots \ldots \ldots \ldots \ldots \ldots \ldots \ldots \ldots \ldots \ldots \ldots$ buldransform_dat

13. angle $\%$ more than a full circle $\ldots \ldots \ldots \ldots \ldots \ldots \ldots \ldots \ldots \ldots \ldots \ldots \ldots \ldots$ convert_rotset

14. angle between nominal and actual lines more than $\%$ degrees . . . .extract_line_cart_bnd, extract_line_cart_unbnd

15. angle between nominal and actual normals more than \% degrees . . . extract_plane_cart

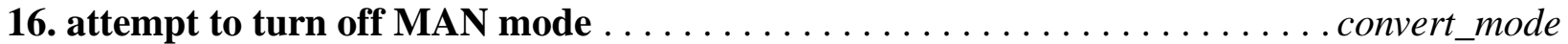

17. axis to align more than $\%$ degrees out of plane . . . . . . . . . find_rotation_aux

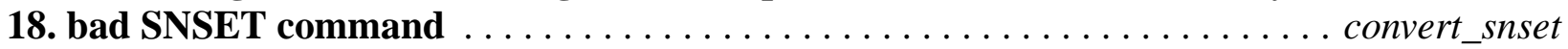

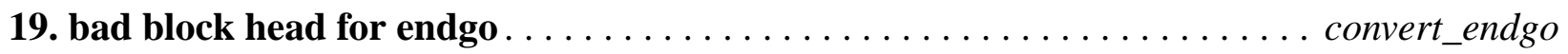

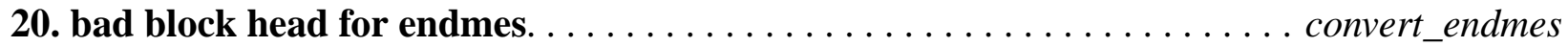

21. bad direction_x $\ldots \ldots \ldots \ldots \ldots \ldots \ldots \ldots \ldots \ldots \ldots \ldots \ldots \ldots \ldots \ldots \ldots$ convert_ptmeas

22. bad direction_y $\ldots \ldots \ldots \ldots \ldots \ldots \ldots \ldots \ldots \ldots \ldots \ldots \ldots \ldots \ldots \ldots \ldots \ldots \ldots$ convert_ptmeas

23. bad direction_z $\ldots \ldots \ldots \ldots \ldots \ldots \ldots \ldots \ldots \ldots \ldots \ldots \ldots \ldots \ldots \ldots \ldots \ldots \ldots$ convert_ptmeas

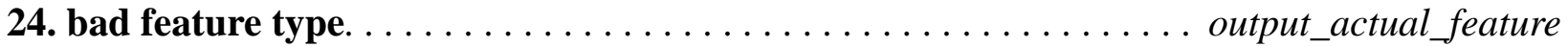

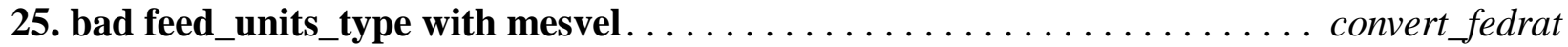

26. bad feed_units_type with posvel $\ldots \ldots \ldots \ldots \ldots \ldots \ldots \ldots \ldots \ldots \ldots \ldots \ldots$ convertfedrat

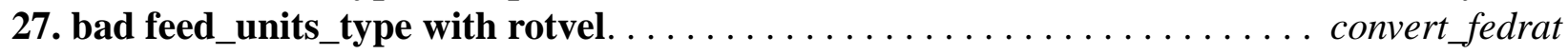

28. bad feed_units_type with scnvel $\ldots \ldots \ldots \ldots \ldots \ldots \ldots \ldots \ldots \ldots \ldots \ldots \ldots \ldots \ldots \ldots$ convert_fedrat

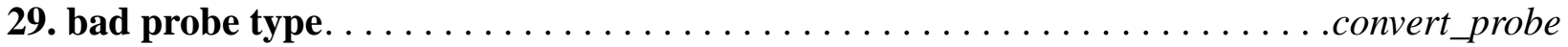

30. bad recall command $\ldots \ldots \ldots \ldots \ldots \ldots \ldots \ldots \ldots \ldots \ldots \ldots \ldots$ convert_recall, output_recall

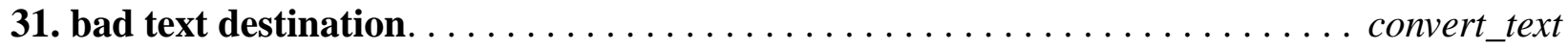

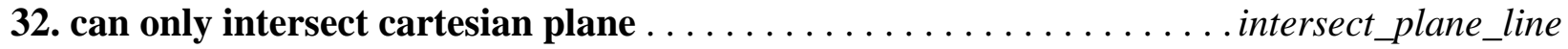

33. can only intersect three cartesian planes $\ldots \ldots \ldots \ldots \ldots \ldots \ldots$ intersect_three_planes

34. cannot align the axis of rotation $\ldots \ldots \ldots \ldots \ldots \ldots \ldots \ldots \ldots \ldots \ldots \ldots \ldots \ldots \ldots \ldots$ find_rotation_aux

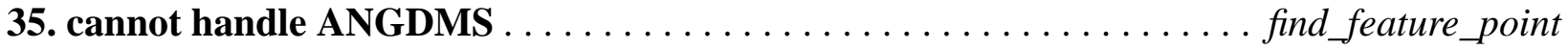

36. cannot handle angle_unit_type $\% \ldots \ldots \ldots \ldots \ldots \ldots \ldots$ convert_rotab, convert_rotset

37. cannot mix $\mathbf{X}$ or $\mathbf{Y}$ reference with $\mathbf{Z}$ distance.............. build_transform_trans

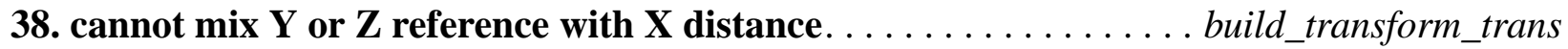

39. cannot $\operatorname{mix} \mathbf{Y}$ or $\mathbf{Z}$ reference with $\mathbf{Y}$ distance. . . . . . . . . build_transform_trans

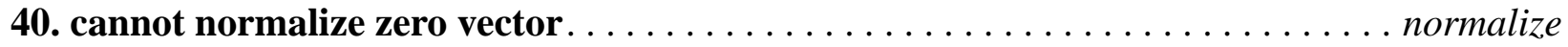

41. circle twin does not exist. . . . . . . . . . . find_feature_point, output_actual_tol_cortol,

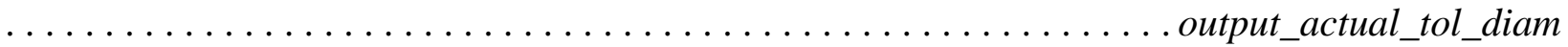

42. current coordinate system transform missing ............. convert_endmes 43. cylndr twin does not exist. .......... output_actual_tol_cortol, output_actual_tol_cylcty, 44. direction vector length not $1 \ldots \ldots \ldots$. extract_cylndr_cart, extract line cart_unbnd 
45. ENDGO position differs from GOTARG position. . . . . . . . . . convert_endgo 46. feature coordinate system transform missing $\ldots \ldots \ldots \ldots \ldots \ldots$ convert_endmes 47. feature is not a cylinder $\ldots \ldots \ldots \ldots \ldots \ldots \ldots \ldots \ldots \ldots \ldots \ldots \ldots$ outpuatul_tol_cylcty 48. feature not a circle. . . . . . . . . . . . . . convert_const_circle, convert_meas_circle 49. feature not a cylndr. . . . . . . . . . . . convert_const_cylndr, convert_meas_cylndr 50. feature not a line . . . . . . . . . . . . . convert_const_line, convert_meas_line 51. feature not a plane .............. convert_const_plane, convert_meas_plane

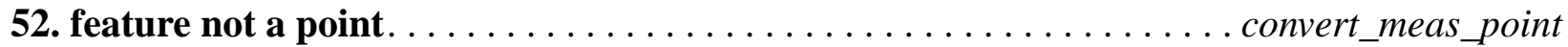

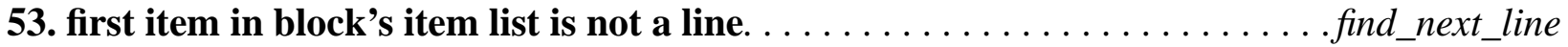

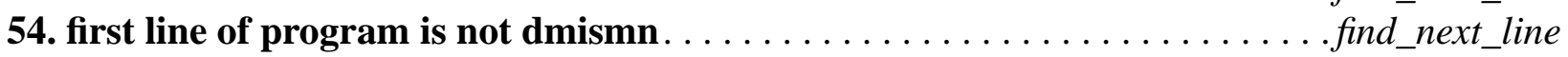

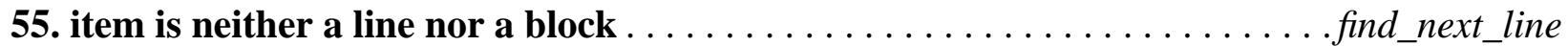
56. item stack is empty $\ldots \ldots \ldots \ldots \ldots \ldots \ldots \ldots \ldots \ldots \ldots \ldots \ldots \ldots \ldots \ldots \ldots \ldots$ find_ne

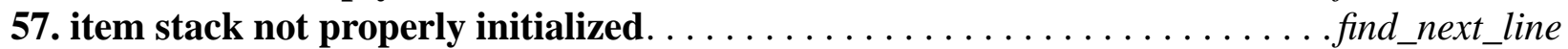

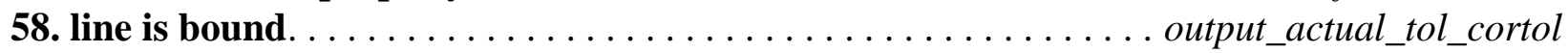

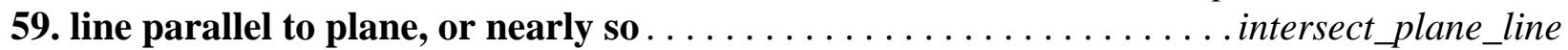
60. line twin does not exist $\ldots \ldots \ldots \ldots \ldots \ldots \ldots \ldots \ldots \ldots \ldots \ldots \ldots \ldots$ outpuactualtol_cortol 61. negative angle $\%$ for rotary table $\ldots \ldots \ldots \ldots \ldots \ldots \ldots \ldots \ldots \ldots \ldots$ convert_rot 62. negative angle for rotary table $\ldots \ldots \ldots \ldots \ldots \ldots \ldots \ldots \ldots \ldots \ldots \ldots \ldots$ convertab 63. negative velocity given in fedrat $\ldots \ldots \ldots \ldots \ldots \ldots \ldots \ldots \ldots \ldots \ldots \ldots \ldots$ convertfedrat 64. normal vector length not $1 \ldots \ldots \ldots \ldots$ extract_circle_cart, extract_plane_cart 65. number of features and actualities differ $\ldots \ldots \ldots \ldots \ldots \ldots \ldots \ldots \ldots$ convert_const 66. plane twin does not exist. . . . . . . . . f find_feature_point, output_actual_tol_cortol 67. planes parallel or nearly so $\ldots \ldots \ldots \ldots \ldots \ldots \ldots \ldots \ldots \ldots \ldots \ldots \ldots \ldots$ intersect_two_planes 68. point twin does not exist . . . . . . . . . . find_feature_point, output_actual_tol_cortol

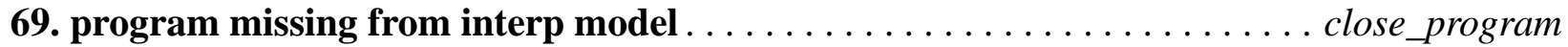

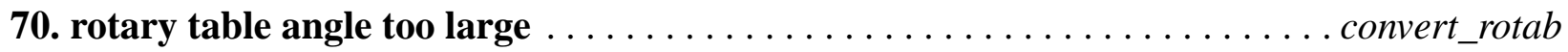
71. scnset type is not DIST or TIME. . . . . $\ldots \ldots \ldots \ldots \ldots \ldots \ldots$ convert_scnset 72. STEP file $\%$ is not readable $\ldots \ldots \ldots \ldots \ldots \ldots \ldots \ldots \ldots \ldots \ldots \ldots \ldots \ldots \ldots \ldots \ldots \ldots \ldots$ 73. too few points $(\%)$ for circle............. convert_const_circle, convert_meas_circle 74. too few points $(\%)$ for cylndr. . . . . . . . . convert_const_cylndr, convert_meas_cylndr

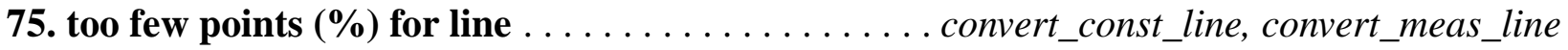
76. too few points $(\%)$ for plane. . . . . . . . . convert_const_plane, convert_meas_plane

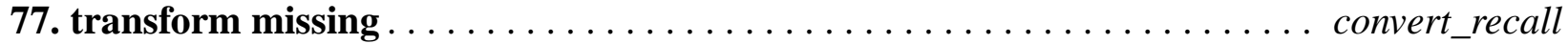
78. twin of circle does not exist $\ldots \ldots \ldots \ldots \ldots \ldots \ldots \ldots \ldots \ldots \ldots \ldots \ldots \ldots \ldots \ldots \ldots$ findfeature_direction

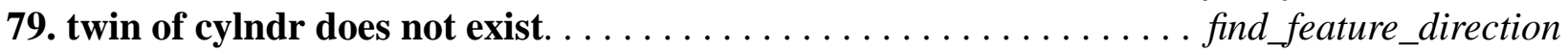

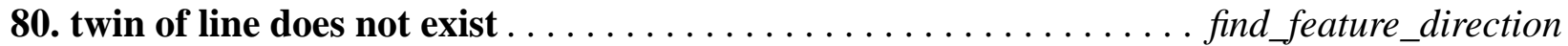
81. twin of plane does not exist $\ldots \ldots \ldots \ldots \ldots \ldots \ldots \ldots \ldots \ldots \ldots \ldots \ldots \ldots \ldots \ldots$ find feature_direction 82. unable to open file $\%$ for reading ...... convert_filnam, convert_to_step, preprocess_dmis

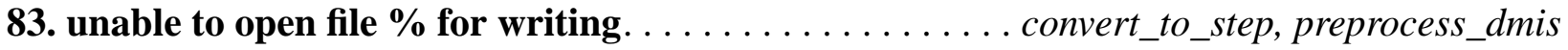
84. unknown angle_unit_type $\ldots \ldots \ldots \ldots \ldots \ldots \ldots \ldots \ldots \ldots \ldots \ldots \ldots \ldots \ldots \ldots \ldots \ldots \ldots \ldots \ldots$

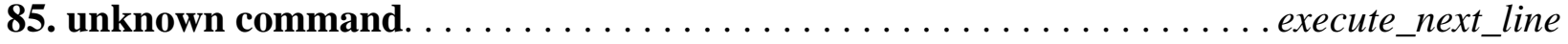

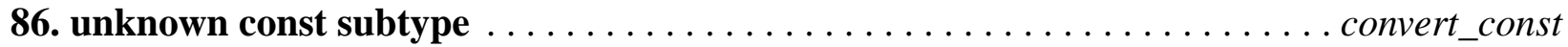

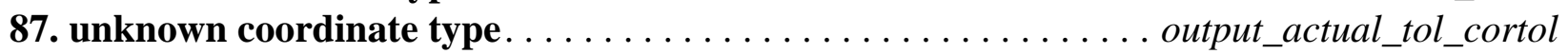

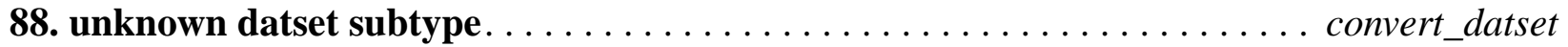
89. unknown definition command $\ldots \ldots \ldots \ldots \ldots \ldots \ldots \ldots \ldots \ldots \ldots \ldots \ldots \ldots \ldots \ldots \ldots \ldots$ convert_dition 90. unknown feature type............ output_actual_tol_cortol, output_actual_tol_diam 
91. unknown feed_set_type $\ldots \ldots \ldots \ldots \ldots \ldots \ldots \ldots \ldots \ldots \ldots \ldots \ldots \ldots \ldots \ldots \ldots \ldots \ldots$ convertfedrat

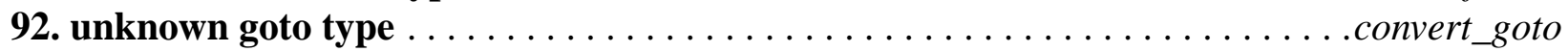

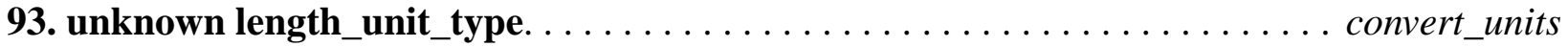

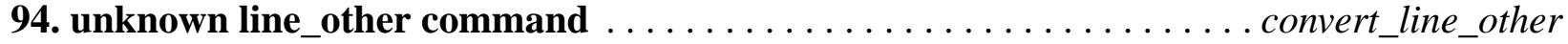

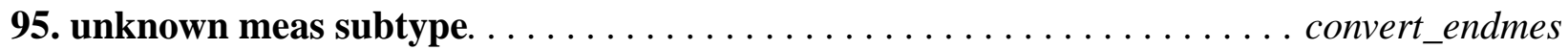

96. unknown on_off type $\ldots \ldots \ldots \ldots \ldots \ldots \ldots \ldots \ldots \ldots \ldots \ldots \ldots$ convert_prcomp

97. unknown point type $\ldots \ldots \ldots \ldots \ldots \ldots \ldots \ldots$ convert_ptmeas, output_actual_tol_cortol

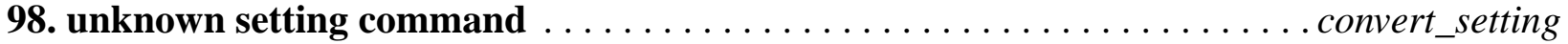

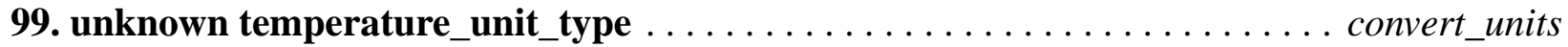

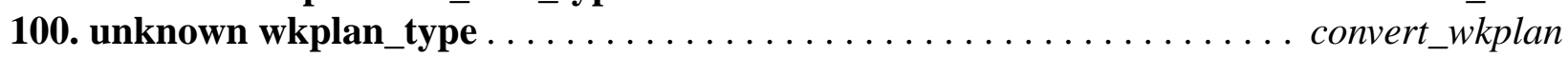

101. unusable feature subtype $\ldots \ldots \ldots \ldots \ldots \ldots \ldots \ldots \ldots \ldots \ldots \ldots \ldots \ldots \ldots \ldots \ldots \ldots$ find_feature_point

102. unusable feature type $\ldots \ldots \ldots \ldots \ldots \ldots \ldots \ldots \ldots \ldots \ldots \ldots \ldots \ldots \ldots \ldots \ldots$ find_feature_direction

103. velocity list has more than one element in fedrat. . . . . . . . . . . . convert_fedrat

104. velocity needed but missing in fedrat $\ldots \ldots \ldots \ldots \ldots \ldots \ldots \ldots$ convert_fedrat

105. velocity provided in fedrat - should not be $\ldots \ldots \ldots \ldots \ldots \ldots \ldots \ldots$ convert_fedrat

106. wrong number $(\%)$ of points measured $\ldots \ldots \ldots \ldots \ldots \ldots \ldots \ldots \ldots \ldots \ldots \ldots \ldots$ convert_endmes

107. wrong number of points $(\%)$ for point - must be $1 \ldots \ldots \ldots$ convert_meas_point

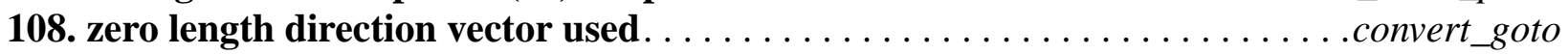




\title{
Appendix F EXPRESS Schema for DMIS
}

\author{
This is an EXPRESS [ISO1] schema for DMIS.
}

\section{F.1 Introduction}

This schema is a primarily a strict EXPRESS representation of the DMIS language. The schema usually leans toward representing DMIS syntax rather than its semantics. This helps make the translation of a program from DMIS to a STEP Part 21 file straightforward. A few items which go beyond a strict representation are discussed in this section.

\section{F.1.1 Line Numbers}

The interpreter keeps track of line numbers from the intermediate file dmis_to_step_temp. The lines of the intermediate file are numbered starting with one. For each line of the intermediate file, an entity instance is written in the Part21 file. The entity instances generated from intermediate file lines all have the line_number attribute because they are all instances of subtypes of the "line" entity defined in this schema. The value of the line_number attribute is assigned (when the Part 21 file is written) as the line number from the intermediate file. The Part21 file also has instances of entities which are not generated from lines of the intermediate file. These instances do not have line numbers.

\section{F.1.2 Optional Reals}

The construct "OPTIONAL REAL" (which was used originally) has been removed because the STEPTools Part21 file reader does not deal well with a missing REAL in an exchange file. It sets the value of the attribute to 0.0 in that case, which is semantically quite different from missing. This schema uses the construct "LIST [0:1] OF REAL" instead of "OPTIONAL REAL". The STEPTools file reader reads empty lists just fine.

\section{F.1.3 Actual Features}

This provides for "actual" features as used in DMIS. An actual feature has the same label as a nominal feature, but its parameters are (in some sense) measured. At least one parameter is derived in part from something measured.

Every feature_definition has a "twin" attribute, the type of which is the same type of feature_definition. The twins of feature_definitions given in the Part21 file will be the actual features. Since none of these exist when the Part21 file is read, the twin attribute is optional in the EXPRESS schema, and its value is $\$$ in the Part21 file. When a feature is measured, the twin of the nominal feature will be created and assigned; this is the actual feature. The twin of the actual feature will be the nominal feature.

Other methods of providing for actual features (not implemented here) include:

1. A list of all nominal feature_definitions could be extracted after the Part21 file is read. A copy of that list would serve as the list of actual features. The values of the attributes of these actual features would be changed when they were measured, and some flag would indicate that a measurement had taken place.

2. Each attribute of each feature_definition could have a value which is a pair (or a list of 1 or 2 
items). The first would be the nominal, and the second would be the actual.

3. Each attribute in a feature_definition could be repeated (a circle would have a nominal_radius attribute and an actual_radius attribute, for example).

Conventions

Almost all entities which correspond directly to a DMIS major word or minor word (or majorminor combination) have been given the name used in the DMIS manual.

Where there are several minor words which are alternatives to one another to follow a major word, an entity for the major word is usually defined and entities which are major-minor combinations are defined as subtypes of it.

In some cases where the semantics of the DMIS manual are clear, but the formal presentation in the manual does not match the semantics well, this schema uses an approach that is closer to the semantics and different from that in the manual.

In a few cases entity types are defined here which do not appear in the manual. These are for defining supertypes of entities which do have corresponding major words in the manual. An example is "definition."

Where some subtype must be instantiated to instantiate a supertype, the supertype is always ABSTRACT (this is normal EXPRESS practice).

\section{F.2 The Schema}

SCHEMA dmis_schema;

$(* * * * * * * * * * * * * * * * * * * * * * * * * * * * * * * * * * * * * * * * * * * * * * * * * * * * * * * * * * * * * * * * * * * * * * * * * *)$

$(*$ TYPES *)

(* all enumeration values are identical to what is in DMIS, except for:

1. the values for operation, which is not defined in DMIS.

2. words which are EXPRESS or STEPTools reserved words. For these the suffix _r is added. This includes: fixed_r, goto_r.

3. words which begin with a minus sign in DMIS. For these the minus sign becomes an underscore and is moved to be the second character. This includes: x_dir, y_dir, z_dir.

4. 2D and 3D, which are illegal enumeration type names in EXPRESS; they are changed to D2 and D3. Some words appear in more than one enumeration type. This does not seem to make a problem when STEPTools express2c++ is used.

$*)$

TYPE angle_unit_type = ENUMERATION OF (angdec, angdms, angrad);

END_TYPE;

TYPE axis_type $=$ ENUMERATION OF (xaxis, yaxis, zaxis, radius, angle); END_TYPE;

TYPE bound_type $=$ ENUMERATION OF (bnd, unbnd);

END_TYPE; 
TYPE dimension_type $=$ ENUMERATION OF $(\mathrm{d} 2, \mathrm{~d} 3) ;(* 2 \mathrm{~d}$ and $3 \mathrm{~d}$ are illegal $*)$ END_TYPE;

TYPE direction_type = ENUMERATION OF (xdir, x_dir, ydir, y_dir, zdir, z_dir); END_TYPE;

TYPE distance_type = ENUMERATION OF (apprch, retrct, search, clrsrf, depth); END_TYPE;

TYPE feed_set_type = ENUMERATION OF (mesvel, posvel, rotvel, scnvel); END_TYPE;

TYPE feed_units_type = ENUMERATION OF (mpm, ipm, rpm, pcent, high, low, defalt $)$; END_TYPE;

TYPE inner_outer_type = ENUMERATION OF (inner, outer); END_TYPE;

TYPE length_unit_type $=$ ENUMERATION OF $(\mathrm{mm}, \mathrm{cm}, \mathrm{m}$, inch, feet $)$; END_TYPE;

TYPE moveability_type = ENUMERATION OF (fixed_r, index); END_TYPE;

TYPE on_off_type = ENUMERATION OF (on, off); END_TYPE;

TYPE operation = ENUMERATION OF (divided_by, minus, plus, raised_to, times); END_TYPE;

TYPE point_type = ENUMERATION OF (cart, pol); END_TYPE;

TYPE rotary_axis_type $=$ ENUMERATION OF (xaxis, yaxis, zaxis); END_TYPE;

TYPE scnset_type = ENUMERATION OF (peck, drag, noncon); END_TYPE;

TYPE temperature_unit_type = ENUMERATION OF (tempf, tempc); END_TYPE;

TYPE text_type = ENUMERATION OF (man, oper, outfil); END_TYPE;

TYPE tol_code $=$ ENUMERATION OF $(1 \mathrm{mc}, \mathrm{mmc}, \mathrm{rfs})$; END_TYPE;

TYPE tolerance_modifier_type = ENUMERATION OF (avg); END_TYPE;

TYPE update_type = ENUMERATION OF (rottot, rotorg, rotnul); END_TYPE;

TYPE wise_absl_type = ENUMERATION OF (cw, ccw, short); END_TYPE; 
TYPE wise_type $=$ ENUMERATION OF $(\mathrm{cw}, \mathrm{ccw})$;

END_TYPE;

TYPE wkplan_type = ENUMERATION OF (xyplan, yzplan, zxplan);

END_TYPE;

$(* * * * * * * * * * * * * * * * * * * * * * * * * * * * * * * * * * * * * * * * * * * * * * * * * * * * * * * * * * * * * * * * * * * * * * * * *)$

$(* \operatorname{ENTITIES~*)}$

ENTITY block

ABSTRACT SUPERTYPE OF (ONEOF (gotarg_block, meas_block))

SUBTYPE OF (dmis_item);

items : LIST [0:?] OF dmis_item;

(* WHERE the first item is not a block *)

END_ENTITY;

ENTITY const_r

ABSTRACT SUPERTYPE OF (ONEOF (const_circle, const_cylndr, const_line,const_plane))

SUBTYPE OF (line_other);

nominal : feat;

first_actual : feat;

features : LIST [1:?] OF feat;

actualities : LIST [1:?] OF BOOLEAN;

(* where the nth actuality goes with the nth feature *)

END_ENTITY;

ENTITY const_circle

SUBTYPE OF (const_r);

END_ENTITY;

ENTITY const_cylndr

SUBTYPE OF (const_r);

END_ENTITY;

ENTITY const_line

SUBTYPE OF (const_r);

END_ENTITY;

ENTITY const_plane

SUBTYPE OF (const_r);

END_ENTITY;

ENTITY datdef

SUBTYPE OF (definition);

associated_feature : feat;

actuality : BOOLEAN;

END_ENTITY;

(* datset

As defined here, datset has a somewhat different format from what is in the manual [CAM-I, p. 113], but this seems to be a clearer way of expressing the same semantics. Only two directions 
are allowed here. The manual allows 3 , but that would over-specify the system.

*)

ENTITY datset

ABSTRACT SUPERTYPE OF (ONEOF (datset_dat, datset_mcs))

SUBTYPE OF (datum_definition);

END_ENTITY;

ENTITY datset_dat

SUBTYPE OF (datset);

direction 1 : direction_type;

datdef1 : datdef;

direction2 : OPTIONAL direction_type;

datdef 2 : OPTIONAL datdef;

x_orig_ref : OPTIONAL datdef;

y_orig_ref : OPTIONAL datdef;

z_orig_ref : OPTIONAL datdef;

(* WHERE direction 1 is not direction2 or opposite to direction2; datdef1 is not datdef2;

the direction of datdef1 is not opposite to that of datdef $2 ; *$ )

END_ENTITY;

ENTITY datset_mcs

SUBTYPE OF (datset);

END_ENTITY;

(* datum_definition

datum_definitions are coordinate systems.

The DMIS DATSET/DAT statement (a subtype of datum definition) makes things difficult because it allows the description of an incompletely defined coordinate system [CAM-I, p. 17 $19,113]$. For example, only the direction of the z-axis and the plane of the z-origin might be defined. The intent of the spec is that DATSET/DAT be followed by ROTATE and TRANS to define the system fully.

If a coordinate system is not fully defined, it may be impossible to carry out subsequent DMIS instructions. Thus, some method of fully defining a coordinate system which is not fully defined by a DATSET/DAT must be adopted. The spec does not include such a method. If the DATSET/ DAT is properly followed by ROTATE and TRANS, any arbitrary choice of data to define the DATSET/DAT system will result in the same coordinate system being defined by the TRANS, so the portion of the program following the TRANS will not be affected. If a feature (or anything else requiring coordinate data) is defined in a DMIS program between the DATSET/DAT and the TRANS, however, its location is affected by the method used to fully define the DATSET/DAT system. Thus, features should not be defined in that part of the program.

If the program fragments shown in the spec on pages 17 and 18 were put together in the given order to make a program, they would be an example of what not to do. The spec does not seem to be aware of the problem just described.

An earlier version of this schema included flags for the parts of a datum_definition that had to be 
invented, but no way was found to propagate the flags reasonably under all subsequent commands.

It is up to the DMIS program generator (human or machine) to avoid the pitfalls made possible with DATSET/DAT.

The transform in a datum_definition is OPTIONAL because it is never defined in a DMIS input file, and it is not created when the Part 21 file is printed (might consider doing that).

*)

ENTITY datum_definition

ABSTRACT SUPERTYPE OF (ONEOF (datset, rotate, trans))

SUBTYPE OF (definition);

transform : OPTIONAL trmatx;

END_ENTITY;

ENTITY definition

ABSTRACT SUPERTYPE OF (ONEOF (datdef, datum_definition, feat, thldef, rotdef, snsdef, tol))

SUBTYPE OF (line);

name : STRING;

END_ENTITY;

(* disply

This corresponds the apparent intent of [CAM-I, p. 332], except that the spec appears to intend to allow multiple vforms. That could be implemented here by substituting "LIST[0:?] OF vform" for "OPTIONAL vform" here. In the spec, OFF "signifies that output will not be sent to any device." That may be accomplished with this entity by explicitly turning everything off.

An instance of a disply entity in a program overrides any and all previous disply entity instances.

*)

ENTITY disply

SUBTYPE OF (line_other);

comm_dmis : BOOLEAN;

comm_form : OPTIONAL vform;

print_dmis : BOOLEAN;

print_form : OPTIONAL vform;

stor_dmis : BOOLEAN;

stor_form : OPTIONAL vform;

term_dmis : BOOLEAN;

term_form : OPTIONAL vform;

END_ENTITY;

ENTITY dmismn

SUBTYPE OF (line_other);

main_name : STRING;

END_ENTITY; 
ENTITY dmis_item

ABSTRACT SUPERTYPE OF (ONEOF (block, line));

END_ENTITY;

ENTITY endfil

SUBTYPE OF (line_other);

END_ENTITY;

ENTITY endgo

SUBTYPE OF (line_other);

END_ENTITY;

ENTITY endmes

SUBTYPE OF (line_other);

END_ENTITY;

ENTITY expression

SUBTYPE OF (expression_or_value);

left : expression_or_value;

op : operation;

right : expression_or_value;

END_ENTITY;

ENTITY expression_or_value

SUPERTYPE OF (ONEOF (expression, value));

END_ENTITY;

(* feat

Conceptually, every feat has a twin, so the twin attribute could be put in here. However, twin needs to be redeclared in each subtype of feat (so each twin has the correct type), so declaring it here is not essential. Including twin here causes express $2 c++$ to emit a warning. Thus, twin is not declared here.

Every feature is defined in some coordinate system (which is a datum_definition), and that is recorded here. See first sentence of [CAM-I, p. $17 \mathrm{sec} 3.5]$.

In the DMIS interpreter, when a feat is read in from the STEP file for the DMIS file, the coordinate system is null. The use of OPTIONAL here allows that. A value is assigned when the feat is executed.

$*)$

ENTITY feat

ABSTRACT SUPERTYPE OF (ONEOF (feat_circle, feat_cylndr, feat_line, feat_plane, feat_point))

SUBTYPE OF (definition);

coordinate_system : OPTIONAL datum_definition;

max_deviation : REAL;

END_ENTITY; 


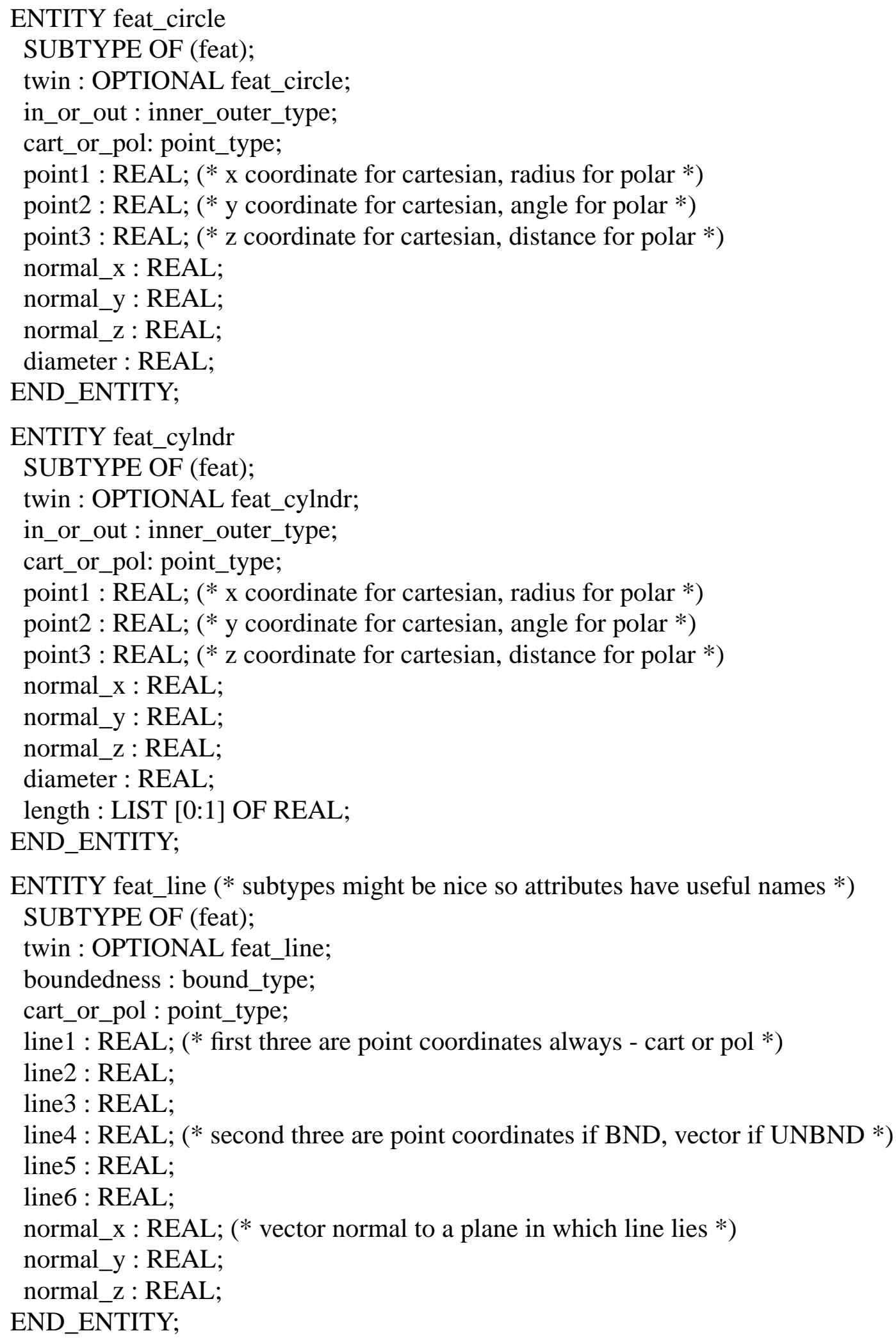


ENTITY feat_plane (* subtypes might be nice so attributes have useful names *)

SUBTYPE OF (feat);

twin : OPTIONAL feat_plane;

cart_or_pol: point_type;

point 1 : REAL; $(* x$ coordinate for cartesian, radius for polar $*)$

point 2 : REAL; (* y coordinate for cartesian, angle for polar $*$ )

point 3 : REAL; $(* \mathrm{z}$ coordinate for cartesian, height for polar $*$ )

normal_i : REAL;

normal_j : REAL;

normal_k : REAL;

END_ENTITY;

ENTITY feat_point

SUBTYPE OF (feat);

twin : OPTIONAL feat_point;

cart_or_pol : point_type;

point 1 : REAL; $(* \mathrm{x}$ coordinate for cartesian, radius for polar *)

point 2 : REAL; (* y coordinate for cartesian, angle for polar $*$ )

point 3 : REAL; $(* \mathrm{z}$ coordinate for cartesian, height for polar $*$ )

normal_i : REAL;

normal_j : REAL;

normal_k: REAL;

END_ENTITY;

ENTITY fedrat

SUBTYPE OF (setting);

to_set : feed_set_type;

feed_units : feed_units_type;

velocity : LIST [0:1] OF REAL;

END_ENTITY;

ENTITY filnam

SUBTYPE OF (line_other);

name_of_file : STRING;

END_ENTITY;

ENTITY gotarg

SUBTYPE OF (line_other);

$\mathrm{x} \_$coordinate : REAL;

y_coordinate : REAL;

z_coordinate : REAL;

END_ENTITY; 
ENTITY gotarg_block

SUBTYPE OF (block);

start : gotarg;

end_block : endgo;

(* WHERE the first item is start and the last item is end_block *)

(* WHERE all other items are goto_r $*$ )

END_ENTITY;

ENTITY goto_abs

SUBTYPE OF (goto_r);

X_coordinate : REAL;

y_coordinate : REAL;

Z_coordinate : REAL;

END_ENTITY;

ENTITY goto_incr

SUBTYPE OF (goto_r);

distance : REAL;

X_direction : REAL;

y_direction : REAL;

z_direction : REAL;

END_ENTITY;

ENTITY goto_r (* goto is a STEPTools reserved word *)

ABSTRACT SUPERTYPE OF (ONEOF (goto_abs, goto_incr))

SUBTYPE OF (meas_block_item);

END_ENTITY;

ENTITY line

ABSTRACT SUPERTYPE OF (ONEOF (definition, line_other, setting))

SUBTYPE OF (dmis_item);

line_number : integer;

END_ENTITY;

ENTITY line_other

ABSTRACT SUPERTYPE OF (ONEOF (const_r, disply, dmismn, endfil, endgo, endmes, filnam, gotarg, meas, meas_block_item, output, recall, rotab, save, snslct, text, units, vform))

SUBTYPE OF (line);

END_ENTITY;

ENTITY meas

ABSTRACT SUPERTYPE OF (ONEOF (meas_circle, meas_cylndr, meas_line,

meas_plane, meas_point))

SUBTYPE OF (line_other);

to_measure : feat;

how_many : INTEGER;

END_ENTITY; 


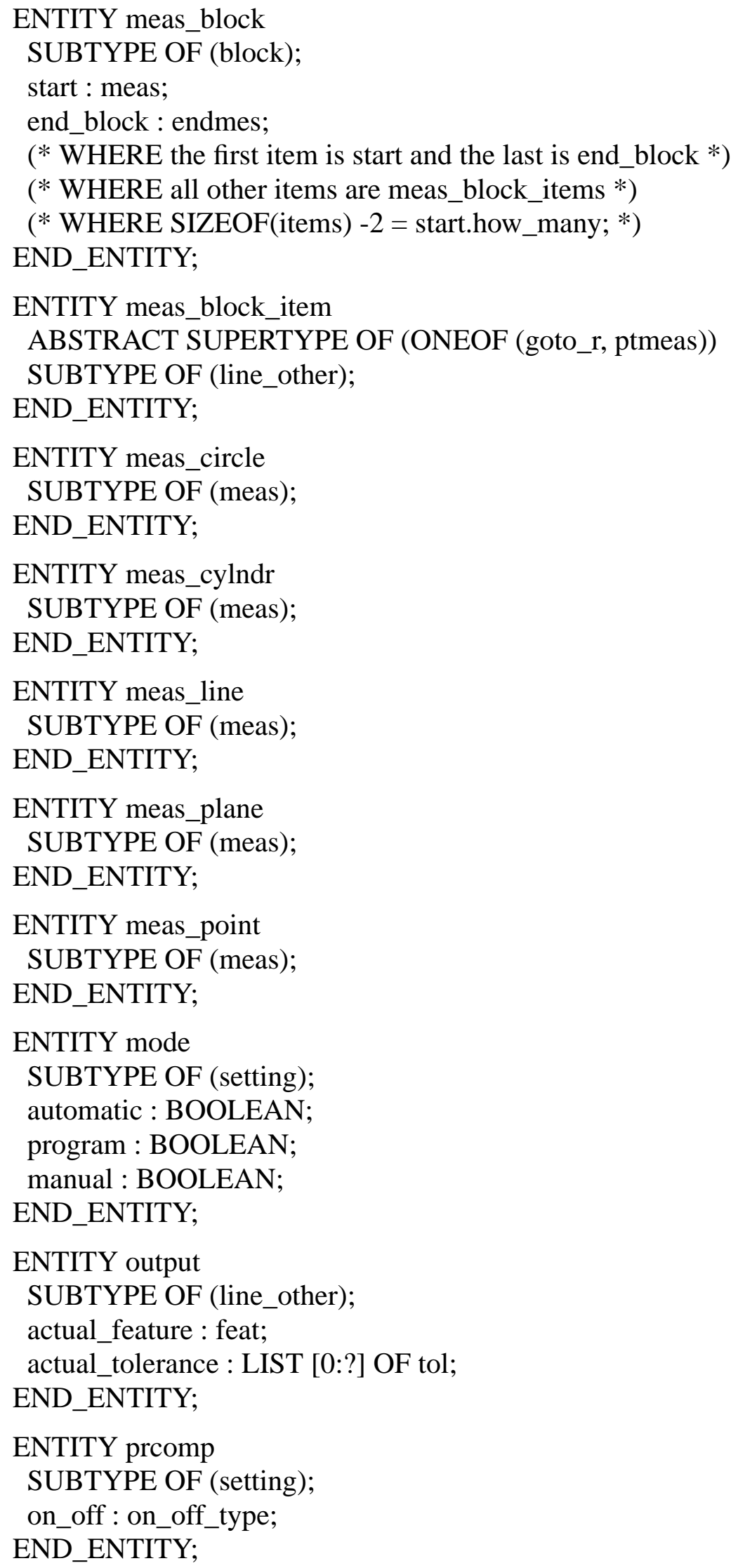




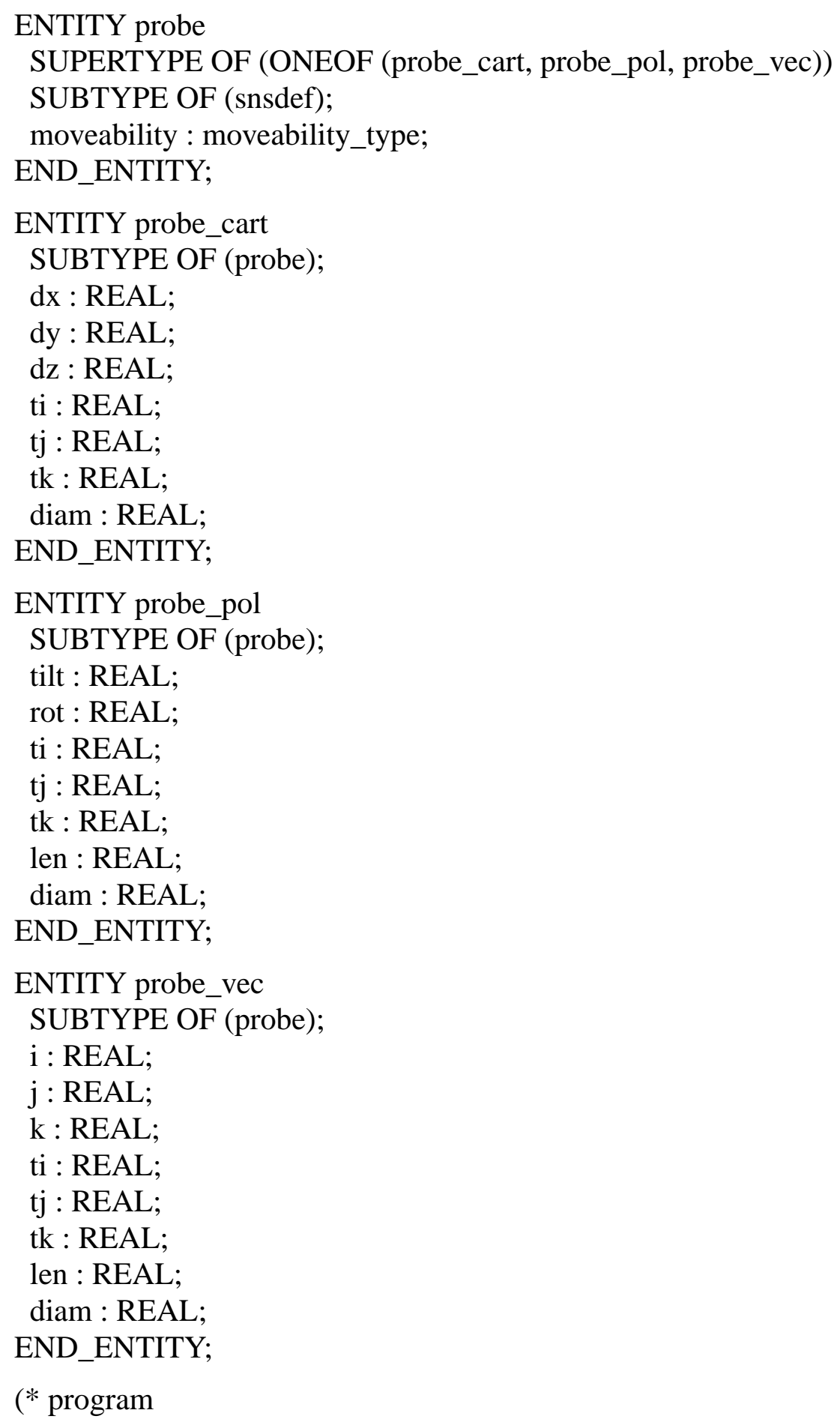

It might be better to rename program to program_block and make it a subtype of block, since conceptually it is a block, and is handled like a block in the software. If this is changed here, the YACC should be changed, too, since then the name is different and the attributes are in a different order and "items" here becomes "item_list".

*) 


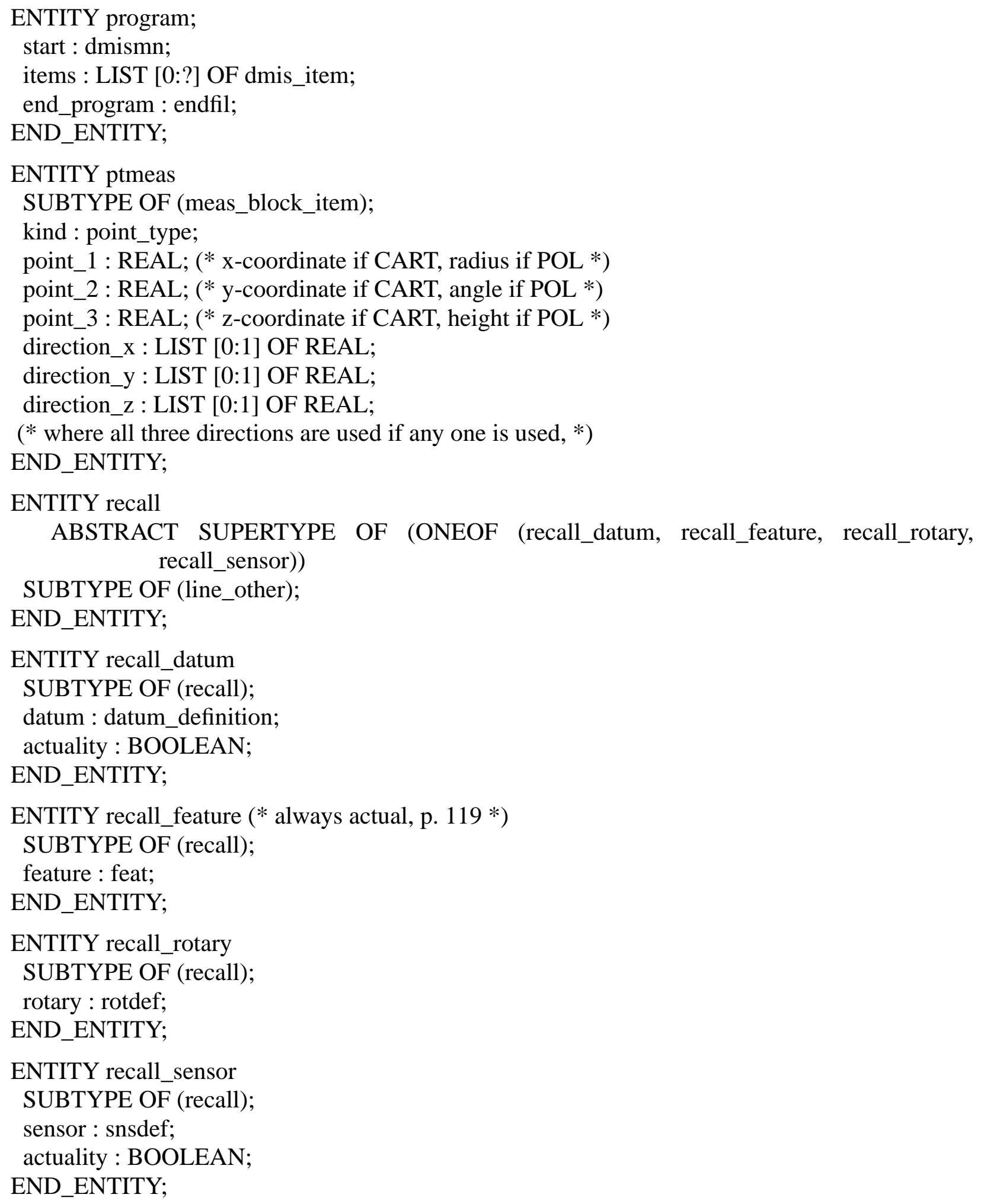




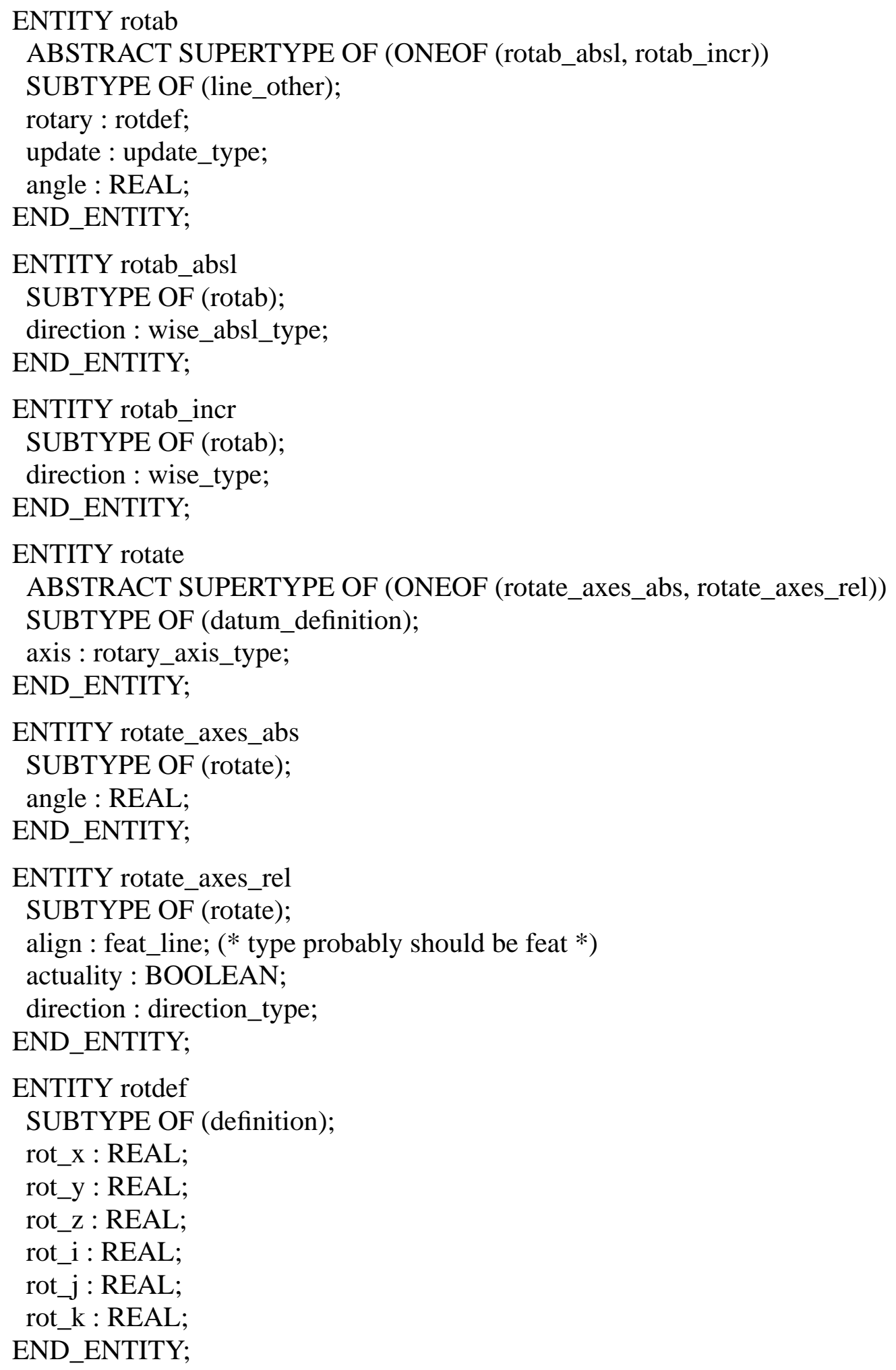




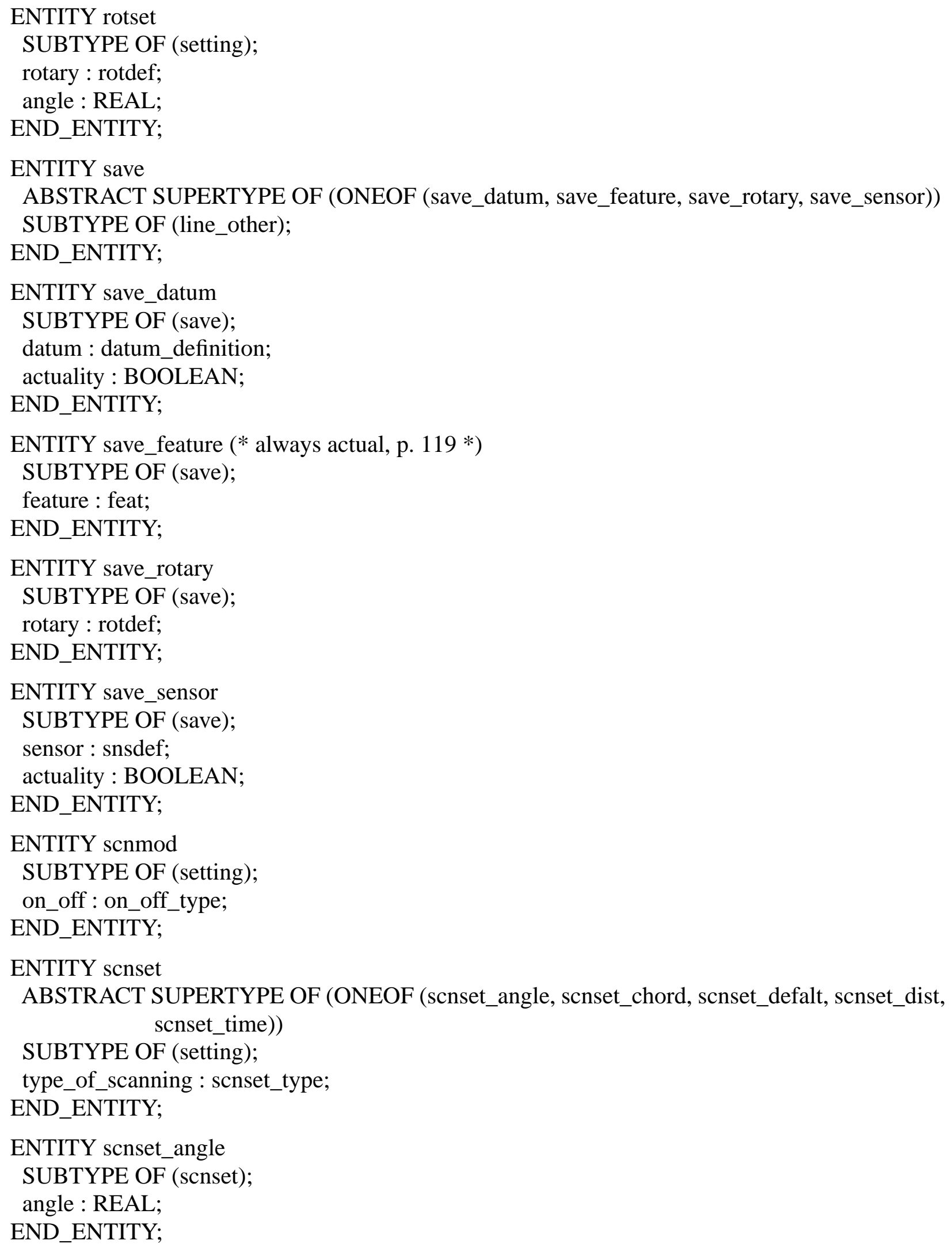




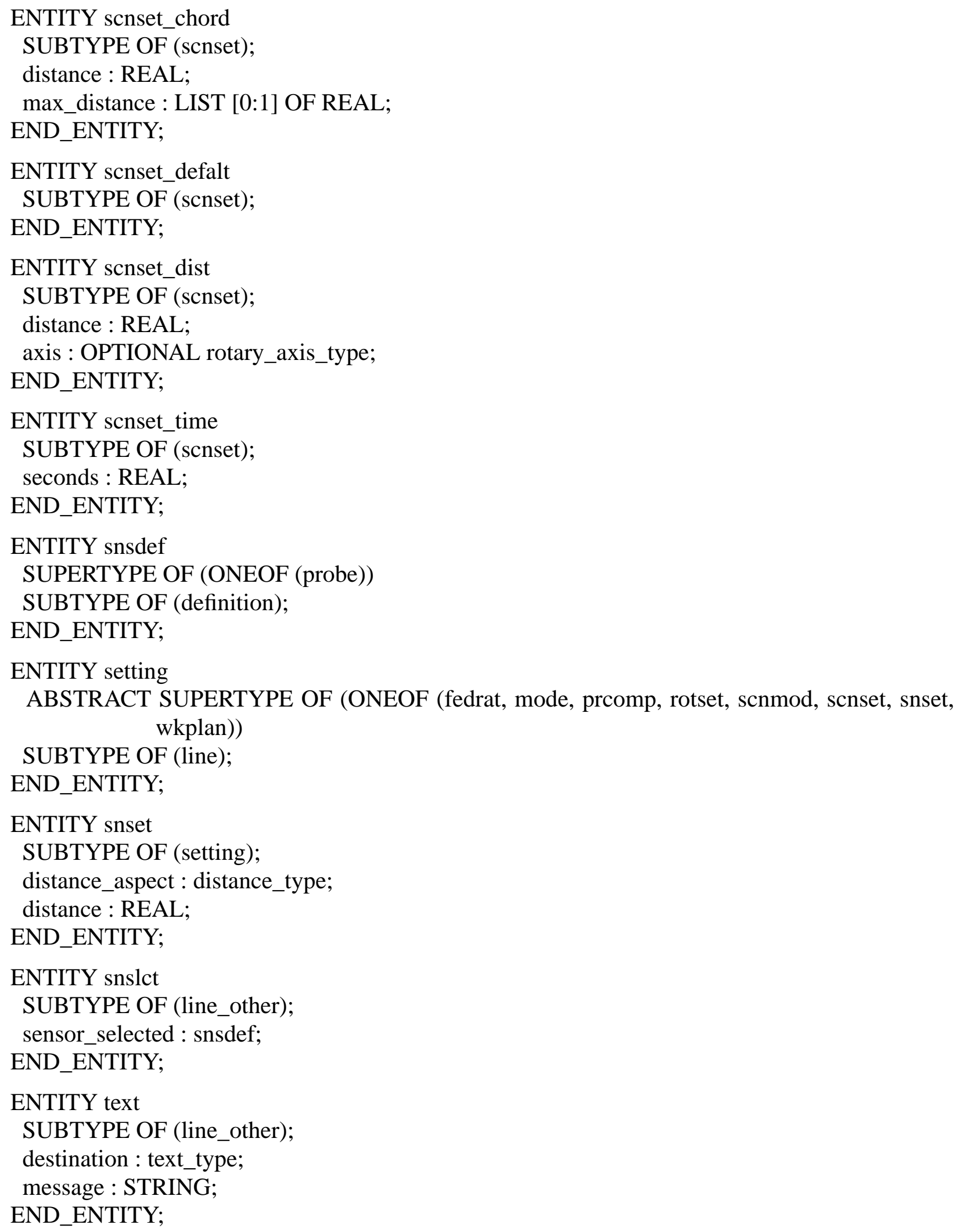




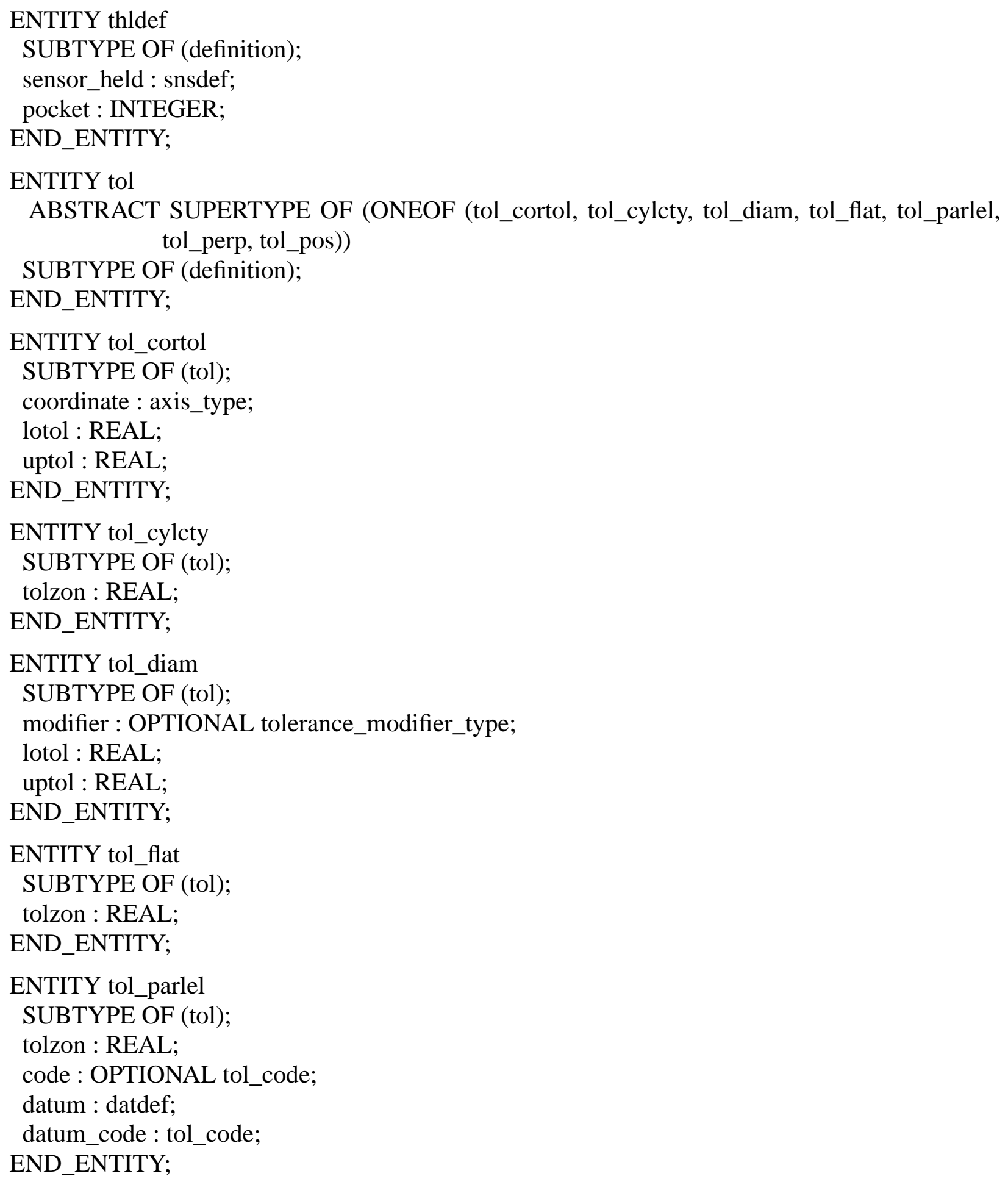




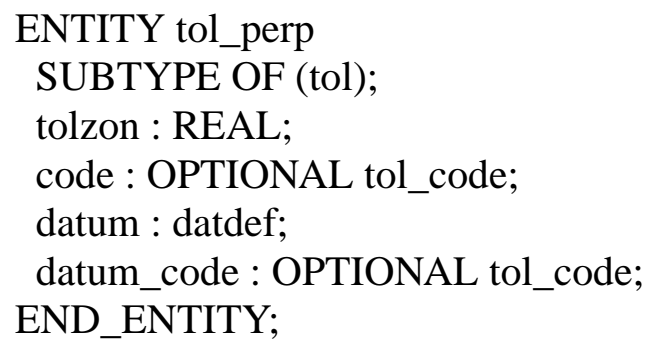

If $x \_$ref is present, $x \_$real indicates whether the feature is actual or not, with zero meaning it is not actual and non-zero meaning it is actual. If $x \_r e f$ is not present, $x \_$real is the actual amount of translation. Similarly for y and $z$.

$*)$ 


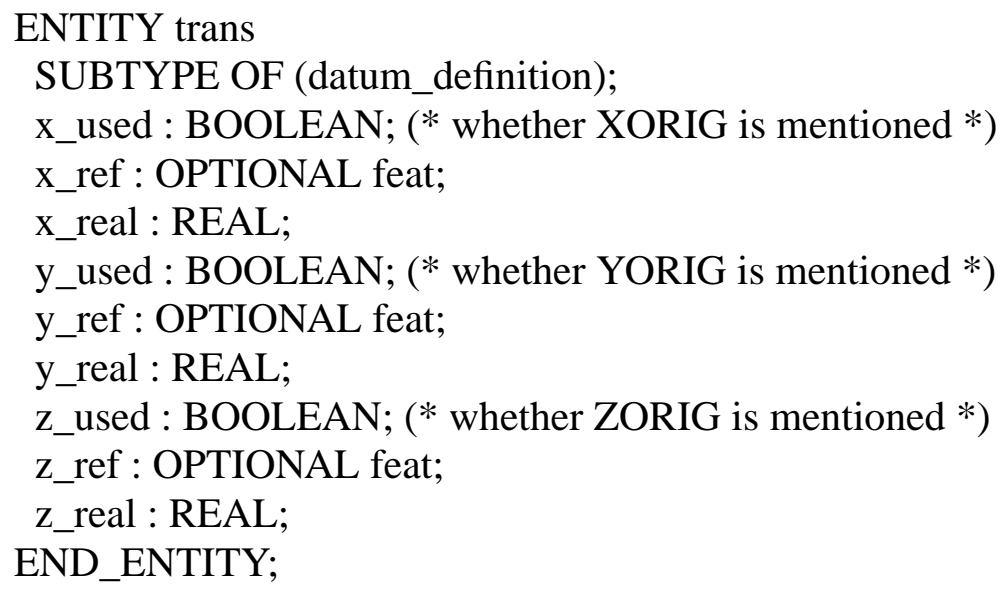

This represents the upper three-fourths of a homogeneous transformation matrix, the bottom line being 0001 . The first three entries are the upper $3 / 4$ of column one, also the rotated $(1,0,0)$. The second three entries are the upper $3 / 4$ of column two, also the rotated $(0,1,0)$. The third three entries are the upper $3 / 4$ of column three, also the rotated $(0,0,1)$. The fourth three entries are the upper $3 / 4$ of column four, also the translation vector. The matrix is:

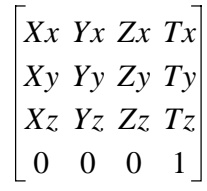

\section{$*)$}

ENTITY trmatx;
$x x$ : REAL;
xy : REAL;
$\mathrm{xz}$ : REAL;
yx : REAL;
yy : REAL;
yz : REAL;
zX : REAL;
zy : REAL;
$\mathrm{zz}$ : REAL;
tx : REAL;
ty : REAL;
tz : REAL;
END_ENTITY; 


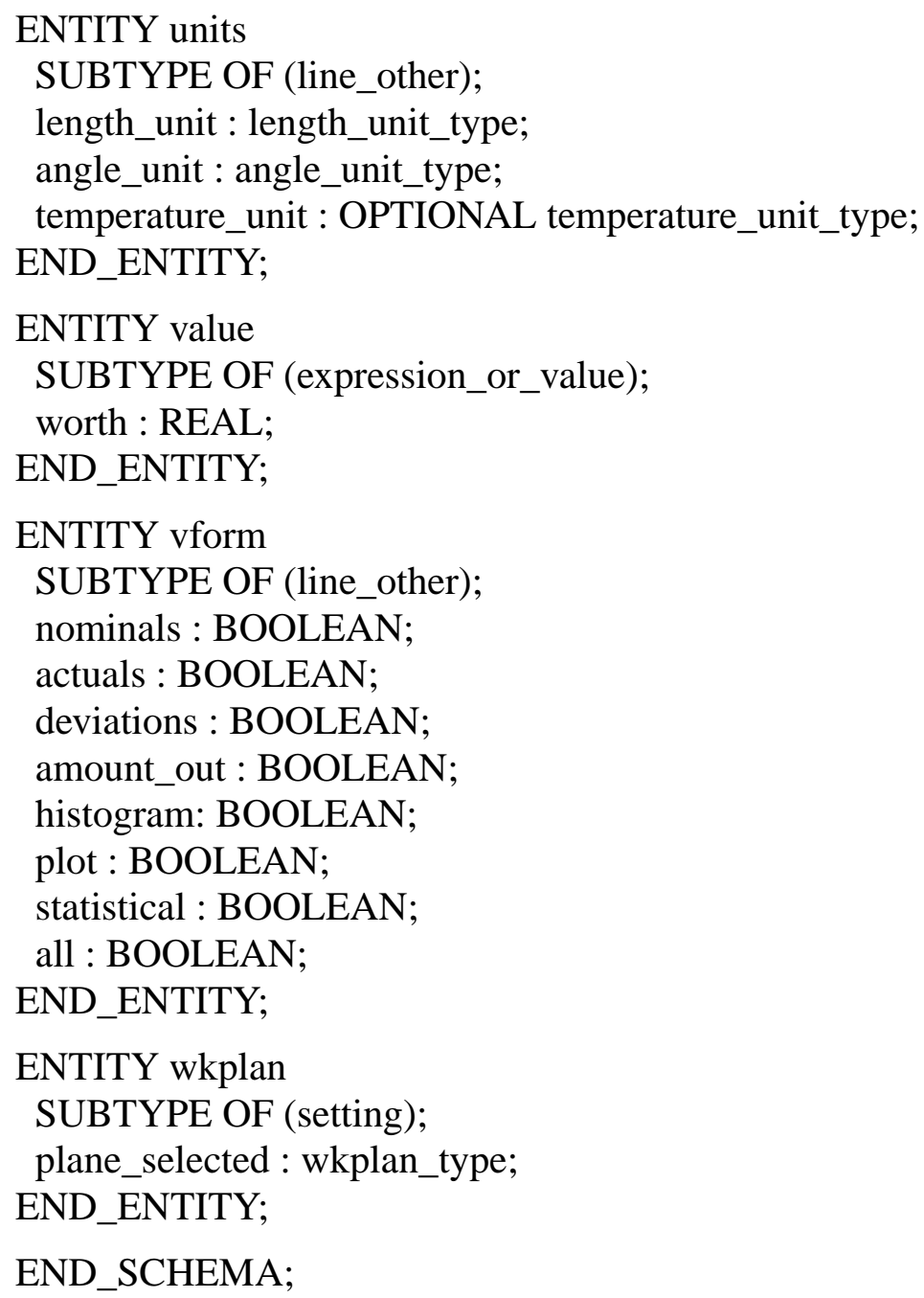




\section{Appendix G YACC Tokens and Grammar Rules}

The following is the YACC tokens and grammar rules used by the DMIS interpreter. The action portions of the rules have been deleted since they are difficult to understand. The full YACC specification with actions included is available on request.

This YACC specification does not deal with comments or line continuations. The interpreter runs the DMIS programs through a pre-processor to remove comments and join lines which are continued. The parser built from this YACC specification takes the pre-processed file as input.

These YACC grammar rules do not include all constraints included in DMIS. Some DMIS statements that are readable under these grammar rules will not be executable because they violate constraints. Almost all constraint violations will be detected by the interpreter and will result in error messages. The error messages are included in Appendix E.

The topmost grammatical unit is "program," so the entire DMIS program is read at once. The first grammar rule is for "program". The rest of the rules are arranged alphabetically.

The lex scanner that works with this is straightforward. White space (tab or space) is allowed between all groups of characters that are tokens. Letters in groups of characters that make tokens are treated the same in upper case or lower case, as stipulated by the DMIS spec. All the tokens are spelled as shown below, except as follows.

1. All tokens which are DMIS major words and take a following slash are spelled with the slash included.

2. BIG_INTEGER is three or more digits (zero to nine) in a row.

3. COMMA_V is a comma followed by a V.

4. LIL_INTEGER is one or two digits in a row.

5. REAL is an optional plus or minus sign followed by zero to many digits, followed by an optional decimal point, followed by zero to many digits (provided that there is at least one digit somewhere in the number).

6. TEXT_STRING is a single quote, followed by any number of characters that are not single quotes, followed by a single quote.

7. X_DIR is spelled “-XDIR".

8. Y_DIR is spelled “-YDIR".

9. Z_DIR is spelled "-ZDIR". 


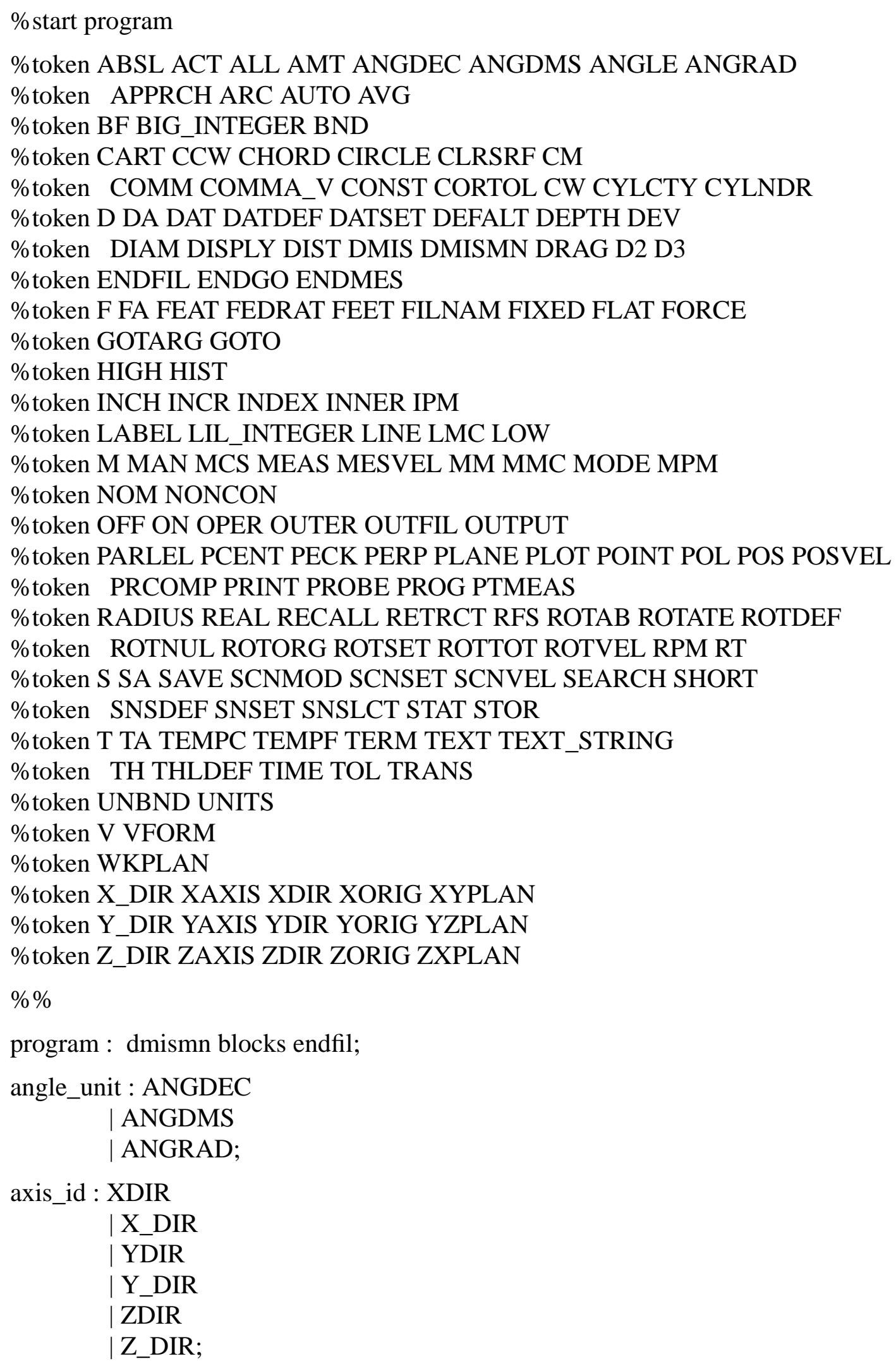


axis_type : ANGLE
| RADIUS
| XAXIS
| YAXIS
ZAXIS;

block : one_liner

| many_liner;

blocks : /* empty */

| blocks block;

bound_type : BND

| UNBND;

const_bf : CONST feature_type ',' F label ',' BF ',' FA label ', f_list 'In';

d_type : D

DA;

datdef : DATDEF f_type label ',' DAT label 'In';

datset : D label '=' DATSET MCS 'In'

| D label '=' DATSET datset_items;

datset_do : datset_dir

| datset_do ',' datset_orig;

datset_dir : XDIR

|X_DIR

Y YDIR

|Y_DIR

ZDIR

|Z_DIR;

datset_items : DAT label ', datset_do 'In'

| DAT label ',' datset_do ',' DAT label ',' datset_do 'In'

| DAT label ', datset_do ',' DAT label ', datset_origs 'In'

| DAT label ',' datset_do ',' DAT label ',' datset_do ', DAT label ',' datset_origs 'In'

| DAT label ',' datset_do ',' DAT label ',' datset_origs ', DAT label ',' datset_origs 'In'

| DAT label ',' datset_do ',' DAT label ',' datset_origs ',' DAT label ',' datset_do 'In';

datset_origs : datset_orig

| datset_origs ',' datset_orig;

datset_orig : XORIG

| YORIG

| ZORIG; 


$$
\begin{aligned}
& \text { definition : datdef } \\
& \qquad \begin{array}{|l|l}
\text { f feat } \\
\text { | probe_definition }
\end{array} \\
& \quad \text { rotdef } \\
& \text { tolerance_definition; }
\end{aligned}
$$

dimension_type : D2

D3;

$$
\begin{array}{cl}
\text { disply } & \text { : DISPLY OFF 'In' } \\
& \mid \text { DISPLY disply_list 'In'; } \\
\text { disply_item : disply_type ', DMIS } \\
\text { | disply_type COMMA_V label } \\
\text { | disply_type ', DMIS COMMA_V label; }
\end{array}
$$

disply_list : disply_item

| disply_list ', disply_item;

disply_type : COMM

| PRINT
| STOR
TERM;

dmismn : DMISMN TEXT_STRING 'In';

endfil : ENDFIL;

endgo : ENDGO;

endmes : ENDMES;

f_item : f_type label;

f_list : f_item

|f_list ', f_item;

f_type : F

|FA;

feat_circle : F label '=' FEAT CIRCLE ', in_out_type ', point_type ', real ',' real ', real ', real ', real ', real ','real 'In';

feat_cylndr : F label '=' FEAT CYLNDR ', in_out_type ', point_type ', real ',' real ', real ', real ',' real ',' real ','real 'In' | F label '=' FEAT CYLNDR ',' in_out_type ', point_type ', real ', real ',' real ',' real ', real ', real ', real ','real 'In';

feat_line : F label '=' FEAT LINE ', bound_type ',' point_type ', real ',' real ',' real ',' real ', real ', real ',' real ',' real ',' real 'In';

feat_plane : F label ‘=' FEAT PLANE ', point_type ',' real ',' real ', real ', real ', real ', real 'In'; 


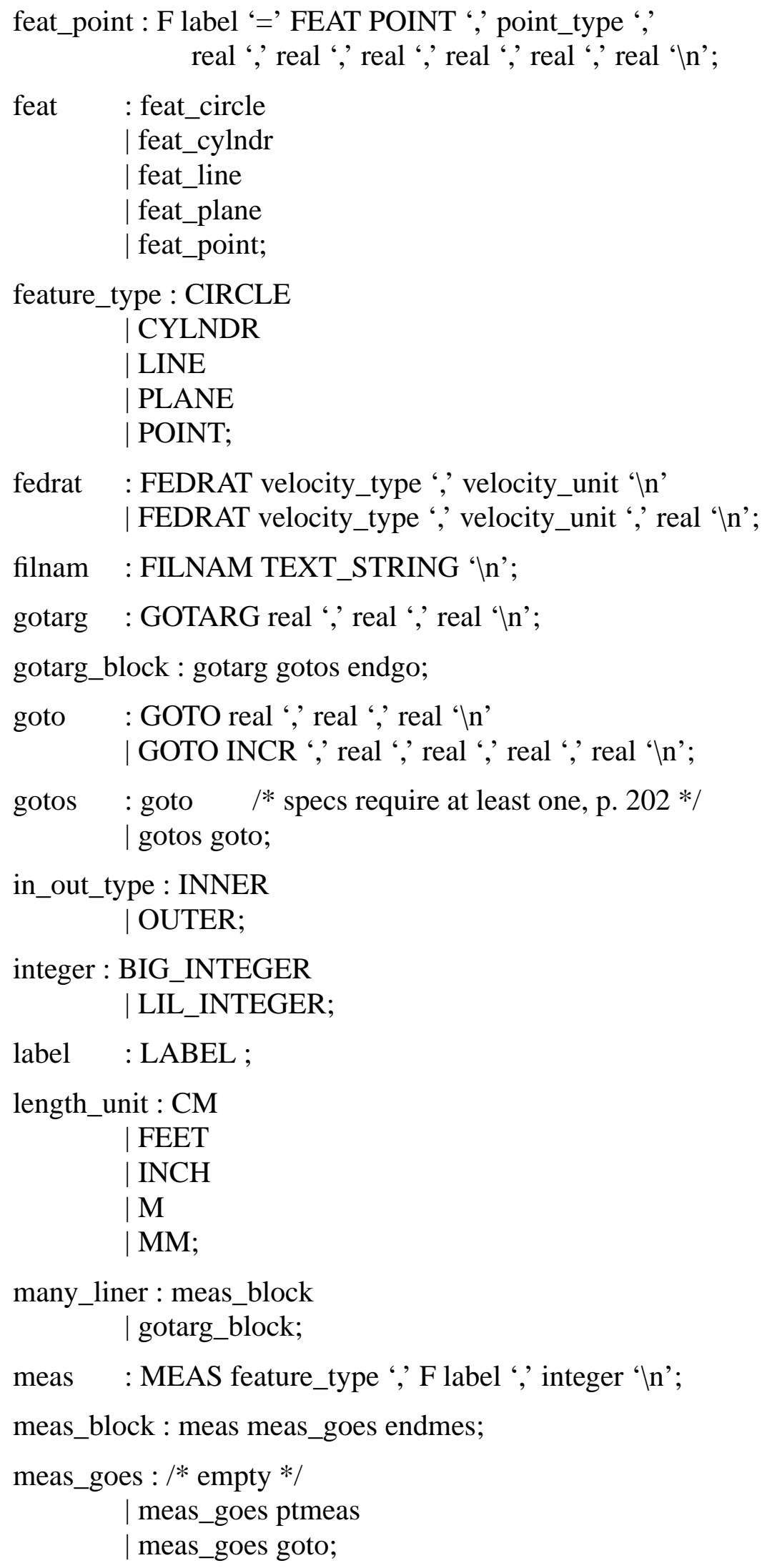




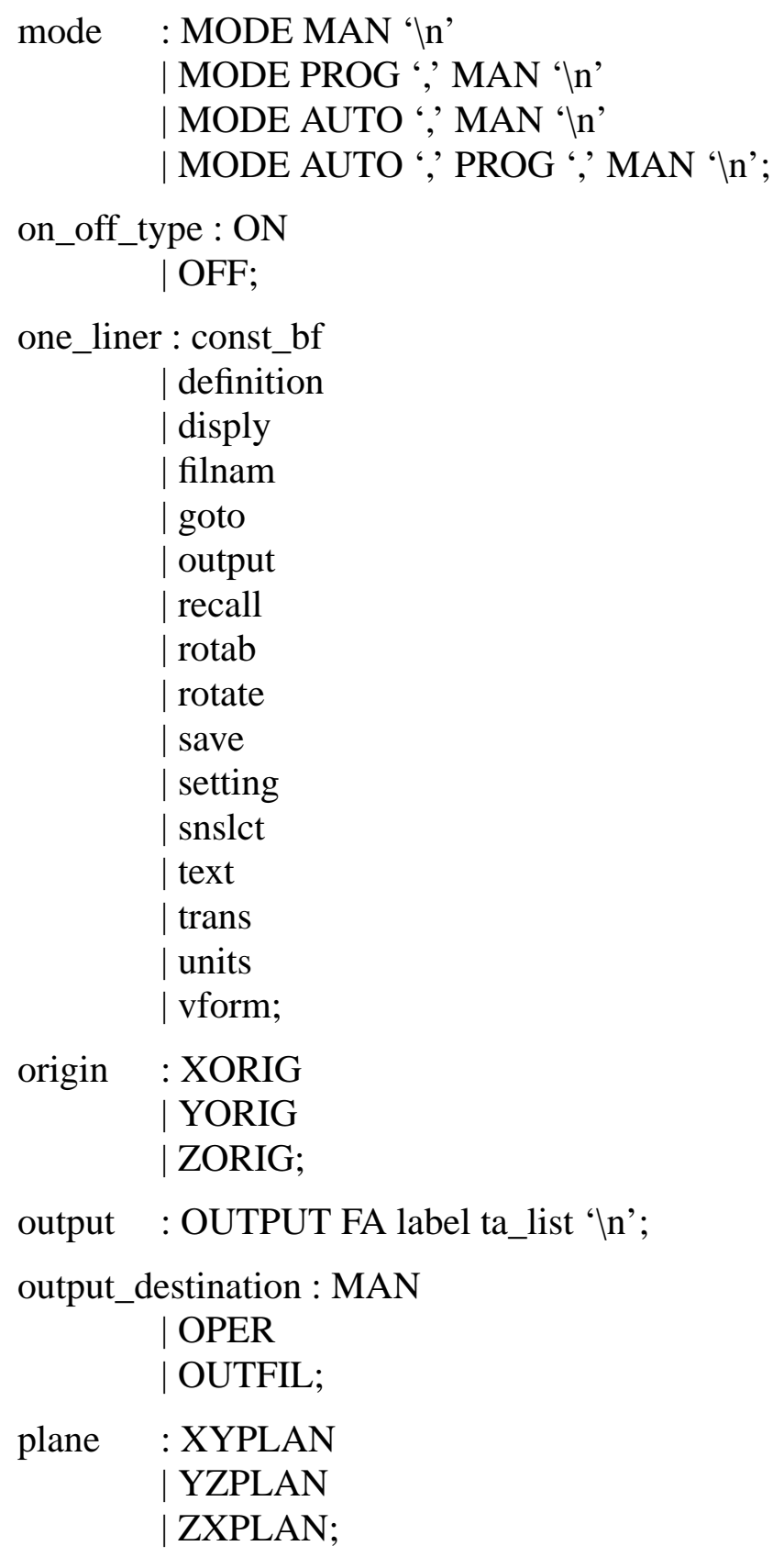




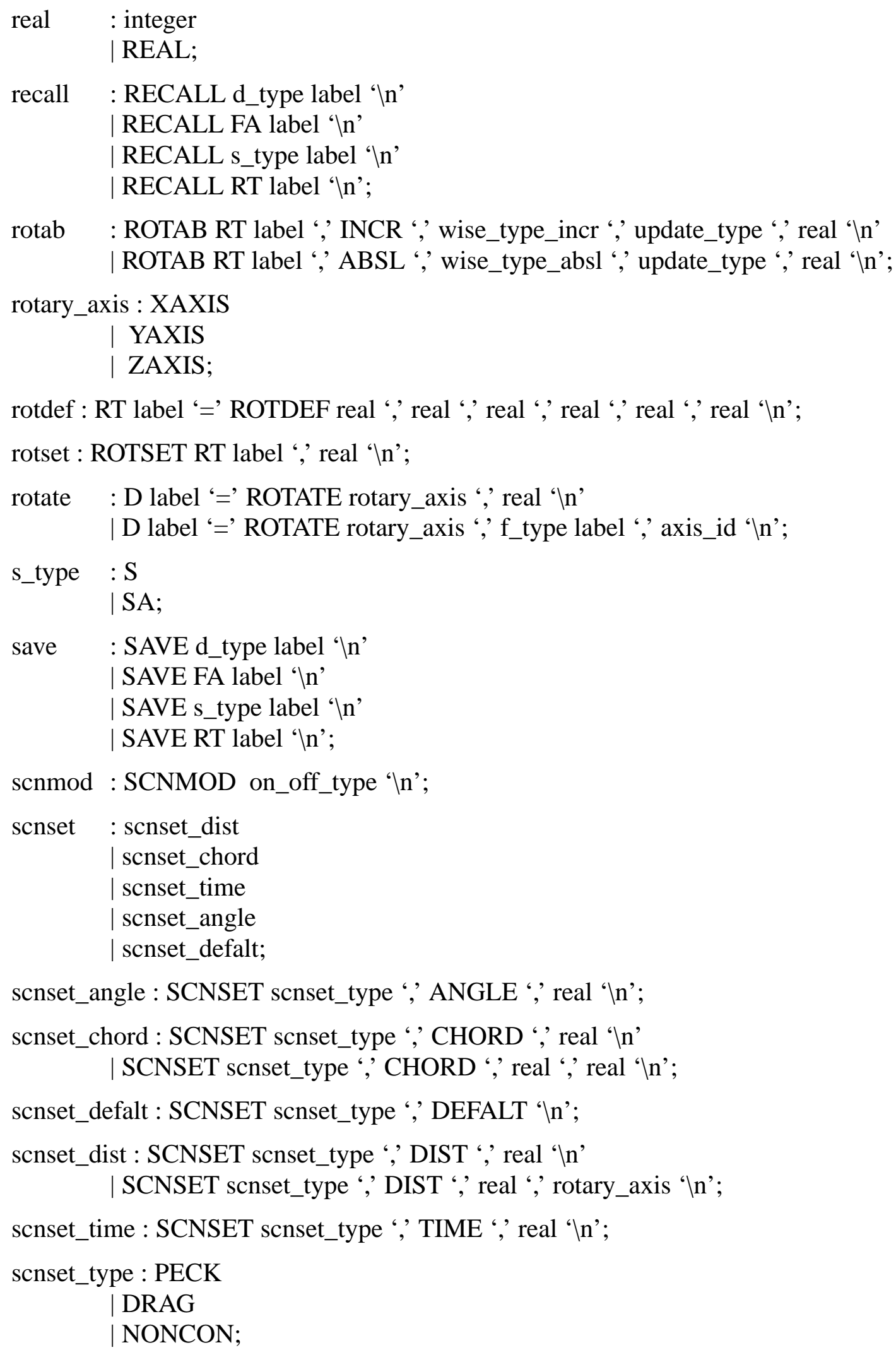




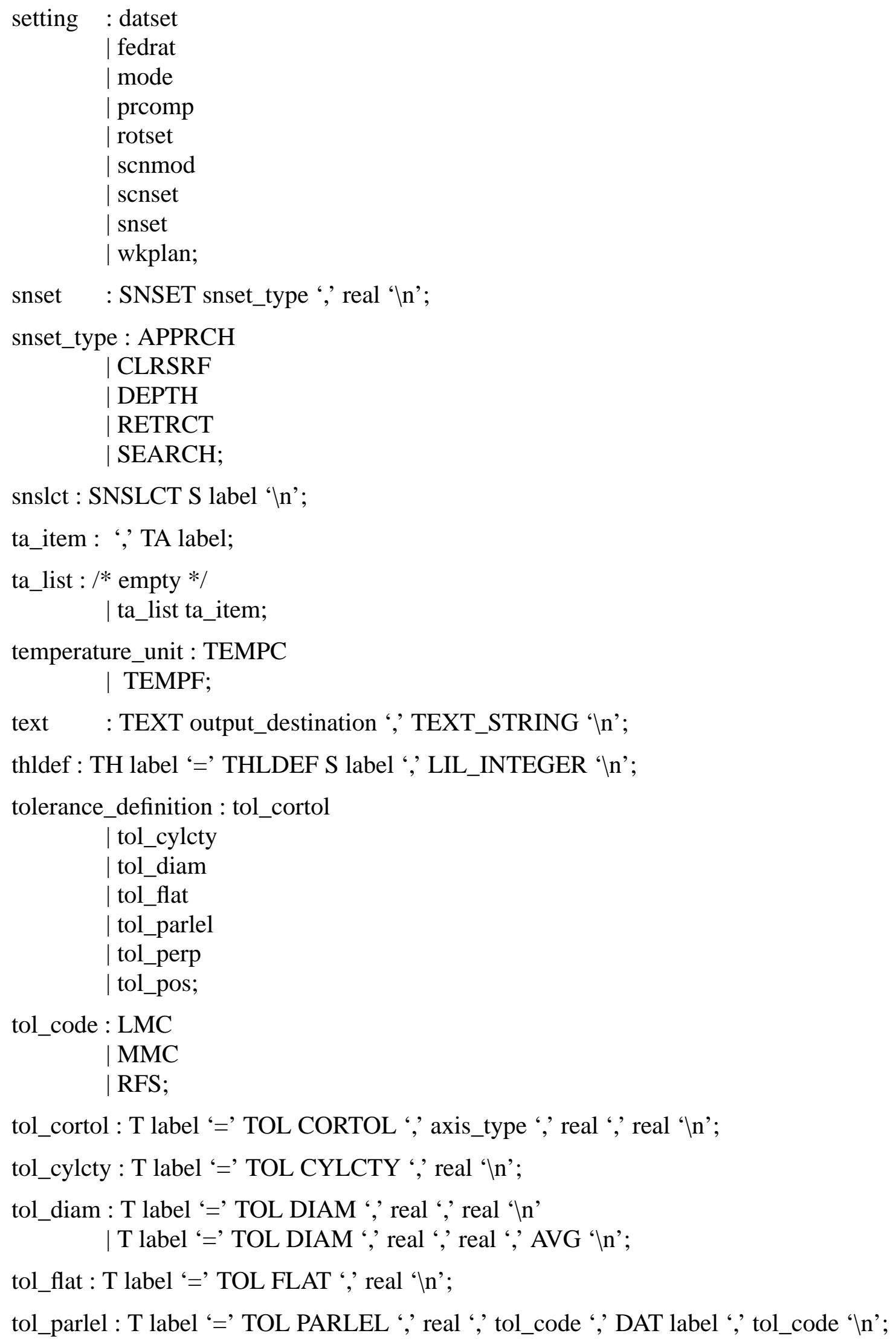




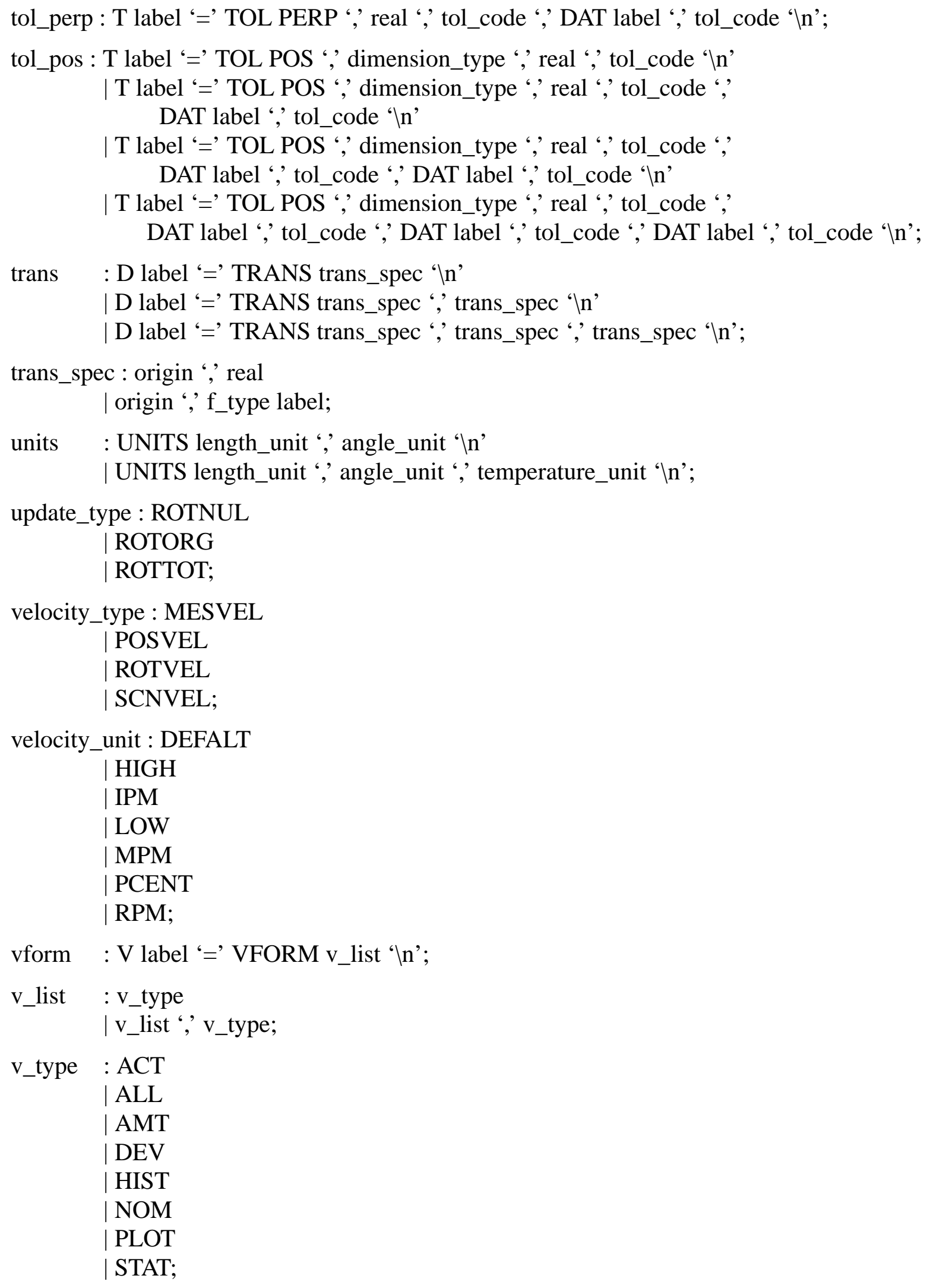


wise_type_absl : CCW

CW

SHORT;

wise_type_incr : CCW

$\mathrm{CW}$;

wkplan : WKPLAN plane 'In';

$\% \%$ 
NIST DMIS Interpreter 